TRANSACTIONS OF THE

AMERICAN MATHEMATICAL SOCIETY

Volume 354, Number 3, Pages 853-900

S 0002-9947(01)02903-8

Article electronically published on October 4, 2001

\title{
STOCHASTIC AVERAGING WITH A FLATTENED \\ HAMILTONIAN: A MARKOV PROCESS ON A STRATIFIED SPACE (A WHISKERED SPHERE)
}

\author{
RICHARD B. SOWERS
}

\begin{abstract}
We consider a random perturbation of a 2-dimensional Hamiltonian ODE. Under an appropriate change of time, we identify a reduced model, which in some aspects is similar to a stochastically averaged model. The novelty of our problem is that the set of critical points of the Hamiltonian has an interior. Thus we can stochastically average outside this set of critical points, but inside we can make no model reduction. The result is a Markov process on a stratified space which looks like a whiskered sphere (i.e, a 2-dimensional sphere with a line attached). At the junction of the sphere and the line, glueing conditions identify the behavior of the Markov process.
\end{abstract}

\section{INTRODUCTION}

One of the principal raisons d'être of "applied mathematics" is in model reduction; i.e., the development of rigorous methods to replace, often in some limiting regime, a complicated system by a simpler, or lower-dimensional one. We here study a model-reduction problem in Markov processes, namely a problem in stochastic averaging.

The underpinning of classical averaging is a separation of time scales; there is a coordinate which slowly varies (often thought of as the energy) and a coordinate which quickly varies (typically thought of as an angle). As the ratio of the fast to slow speed increases, it becomes possible to in a sense fix the slowly varying coordinate and carry out a long-term average in the quickly varying component. A simple example of such a system is a Hamiltonian system with small perturbations. The slowly varying coordinate is the value of the Hamiltonian and the quickly varying coordinate is the position (or angle) in the appropriate level set of the Hamiltonian. One can seek a reduced model for the slow variable by first rescaling time so that the variation of the slow variable is of order one. As the fast motion

Received by the editors September 7, 2000 and, in revised form, June 01, 2001.

1991 Mathematics Subject Classification. Primary 60F17; Secondary 37J40, 58A35, 60J35.

Key words and phrases. Markov processes, stochastic averaging, stratified space.

This work was supported by NSF DMS 9615877 and NSF DMS 0071484. The author would also like to thank Professor Sri Namachchivaya of the Department of Aeronautical and Astronautical Engineering at the University of Illinois at Urbana-Champaign for the seemingly infinite time he donated to discussing the contents of this paper and without whose interest this subject would not have been considered. The author would also like to thank Professor Eugene Lerman of the Department of Mathematics at the University of Illinois at Urbana-Champaign for several helpful discussions about stratified spaces. 
becomes faster, the behavior of the slow variable can (often) be described via a closed set of equations (without reference to the fast variable).

The nature and complexity of the reduced or averaged process depends on the complexity of the Hamiltonian $H$; roughly, the reduced process takes values in the space of orbits of the fast motion. When $H$ is fairly simple, viz., something like a paraboloid with a single isolated elliptic critical point, the reduced process is simply a Markov process on a line - a classical result [15. However, this machinery has only recently been extended to handle more complicated Hamiltonians; e.g., Hamiltonians with a finite collection of distinct minima and saddle points; in this case the reduced Markov process takes its values in a graph which encodes the topology of the level sets of $H$ [8, 9, 10. A common assumption in all studies up to now is that $H$ is nondegenerate at its critical points (i.e., the Hessian of $H$ at its critical points is nondegenerate). Our goal here is to investigate what happens when this last assumption is violently removed; i.e., when the set of critical points actually has an interior. Outside of the set of critical points, standard stochastic averaging can be used to asymptotically reduce the dimension. Inside the set of critical points, however, no dimensional reduction can occur. One must then glue these two spaces and the corresponding processes together. In the simple 2-dimensional example which we consider here, we will see that the result is a Markov process on a whiskered sphere (a sphere with a line attached). More mathematically, the process takes place on a stratified space. The Markov process on the stratified space naturally involves the original generator inside the set of critical points and the averaged generator outside the set of critical points, but also involves a glueing condition at the junction.

Stochastic averaging has a long history. Stratonovich [23] and Gikhman [11] developed some of the first averaging results. Completely rigorous arguments involving diffusion processes were given by Khas'minskii [14, 15]. Several authors [3, 4, 18, 19] have subsequently developed the classical theory in different directions under various less stringent assumptions and with real noise. The formulation of stochastic averaging using the martingale problem was developed by Papanicolaou and Kohler [20]; we too shall use the martingale problem formulation. All of these efforts involved Hamiltonians with a single nondegenerate minimum. As we mentioned above, Freidlin and Wentzell [10] gave the first successful rigorous analysis of a system involving a Hamiltonian with multiple minima and saddle points (although there were prior efforts in this direction by Neishtadt [16] and Wolansky [25]).

Our effort here appears to be one of the first studies of a Markov process on a stratified space with a dimensional discontinuity. The only other result we have been able to find about a process with such a dimensional discontinuity is in the recent work of Burdzy and Bass 2 which introduced a "fiber" Brownian motion to study the "hot spots" problem. There is a vast literature about diffusions on sets which locally look like Euclidean space (i.e., differentiable manifolds); part of the novelty of this work is that one of the model spaces for our stratified space is the union of a line and a plane; see Figure 2 .

We hope that some of the techniques developed here have a somewhat wider applicability. At the heart of our efforts is a certain boundary-layer analysis. Since we are interested in a fast drift and diffusion of order one, this boundary analysis naturally can be phrased as a singular perturbation problem. Some classical work 
by Khasminskii 13 suggests a coordinate transformation which makes the dominant part of this singular perturbation problem explicitly solvable. The singular perturbation analysis using Khasminskii's coordinates appears to be a common way to study various stochastic averaging problems involving glueing conditions.

\section{Problem Statement and Main Result}

Our problem will be on $\mathbb{R}^{2}$. For any function $F \in C^{1}\left(\mathbb{R}^{2}\right)$, we define the vector field (or more accurately derivation) $\bar{\nabla} F$ as

$$
\begin{aligned}
((\bar{\nabla} F) \varphi)\left(x_{1}, x_{2}\right) \stackrel{\text { def }}{=} \frac{\partial F}{\partial x_{2}}\left(x_{1}, x_{2}\right) \frac{\partial \varphi}{\partial x_{1}}\left(x_{1}, x_{2}\right)-\frac{\partial F}{\partial x_{1}}\left(x_{1}, x_{2}\right) \frac{\partial \varphi}{\partial x_{2}}\left(x_{1}, x_{2}\right), & \\
\varphi & \in C^{\infty}\left(\mathbb{R}^{2}\right),\left(x_{1}, x_{2}\right) \in \mathbb{R}^{2},
\end{aligned}
$$

(i.e., $\bar{\nabla} F$ is the symplectic gradient of $F$ with the usual symplectic structure on $\mathbb{R}^{2}$ ). For the purposes of discussion, let's fix a Hamiltonian function $H$, on which we for the moment make minimal assumptions:

Assumption 2.1 (Hamiltonian). Let $H$ be in $C^{2}\left(\mathbb{R}^{2}\right)$.

We will later make some further restrictions on $H$. The generator of the fast motion will be $\varepsilon^{-2} \bar{\nabla} H$. We want to perturb this by a diffusive motion.

Assumption 2.2 (Diffusion Generator and Bracket). Let $\mathscr{L}$ be a second-order partial differential operator of the form

$$
(\mathscr{L} f)\left(x_{1}, x_{2}\right) \stackrel{\text { def }}{=} \frac{1}{2} \sum_{i, j \in\{1,2\}} a_{i, j}\left(x_{1}, x_{2}\right) \frac{\partial^{2} f}{\partial x_{i} \partial x_{j}}\left(x_{1}, x_{2}\right)+\sum_{i \in\{1,2\}} b_{i}\left(x_{1}, x_{2}\right) \frac{\partial f}{\partial x_{i}}\left(x_{1}, x_{2}\right)
$$

for all $f \in C^{2}\left(\mathbb{R}^{2}\right)$ and $\left(x_{1}, x_{2}\right) \in \mathbb{R}^{2}$, where the $a_{i, j}$ 's and $b_{i}$ 's are $C^{\infty}$ functions on $\mathbb{R}^{2}$. Define the bilinear form $\langle\cdot, \cdot\rangle$ on $T^{*} \mathbb{R}^{2}$ via the formula

$$
\begin{aligned}
\langle d f, d g\rangle\left(x_{1}, x_{2}\right)= & \mathscr{L}(f g)\left(x_{1}, x_{2}\right)-f\left(x_{1}, x_{2}\right)(\mathscr{L} g)\left(x_{1}, x_{2}\right) \\
& -g\left(x_{1}, x_{2}\right)(\mathscr{L} f)\left(x_{1}, x_{2}\right) \\
= & \sum_{i, j \in\{1,2\}} a_{i, j}\left(x_{1}, x_{2}\right) \frac{\partial f}{\partial x_{i}}\left(x_{1}, x_{2}\right) \frac{\partial g}{\partial x_{j}}\left(x_{1}, x_{2}\right),
\end{aligned}
$$

which we assume to hold for all $f$ and $g$ in $C^{2}\left(\mathbb{R}^{2}\right)$ and all $\left(x_{1}, x_{2}\right) \in \mathbb{R}^{2}$. We require that $\mathscr{L}$ be strongly elliptic; i.e.,

$$
\langle d f, d f\rangle(x)>0
$$

for all $x \in \mathbb{R}^{2}$ and all $f \in C^{2}\left(\mathbb{R}^{2}\right)$ with $d f(x) \neq 0$.

We note that if $f \in C^{2}\left(\mathbb{R}^{2}\right)$ and $\Phi \in C^{2}(\mathbb{R})$, then

$$
\mathscr{L}(\Phi \circ f)(x)=\dot{\Phi}(f(x))(\mathscr{L} f)(x)+\frac{1}{2} \ddot{\Phi}(f(x))\langle d f, d f\rangle(x)
$$

for all $x \in \mathbb{R}^{2}$. We will consider the Markov process on $\mathbb{R}^{2}$ whose generator is

$$
\mathscr{L}^{\varepsilon} \stackrel{\text { def }}{=} \mathscr{L}+\frac{1}{\varepsilon^{2}} \bar{\nabla} H
$$

(with domain $\mathscr{D}\left(\mathscr{L}^{\varepsilon}\right) \supset C^{2}\left(\mathbb{R}^{2}\right)$ ). We will construct this Markov process in a canonical way, via the martingale problem [5] 24]. Define the event space $\Omega \stackrel{\text { def }}{=}$ $C\left([0, \infty) ; \mathbb{R}^{2}\right)$. Define the coordinate functions $X_{t}(\omega) \stackrel{\text { def }}{=} \omega(t)$ for all $t \geq 0$ and all 
$\omega \in \Omega$. For each $t \geq 0$, define $\mathscr{F}_{t} \stackrel{\text { def }}{=} \sigma\left\{X_{s} ; 0 \leq s \leq t\right\}$ and define a sigma-algebra on $\Omega$ by $\mathscr{F} \stackrel{\text { def }}{=} \bigvee_{t \geq 0} \mathscr{F}_{t}$. Note that we have made no assumptions on the structure of $H$ at infinity, nor do we want to. Fix an energy level $\bar{H}>0$ and define the set

$$
\mathbf{S} \stackrel{\text { def }}{=}\left\{x \in \mathbb{R}^{2}: H(x)<\bar{H}\right\}
$$

and let $\mathfrak{e}$ be the first time that $X$ leaves $\mathbf{S}$; i.e.,

$$
\mathfrak{e} \stackrel{\text { def }}{=} \inf \left\{t \geq 0 ; H\left(X_{t}\right) \geq \bar{H}\right\} \text {. }
$$

We can now define our principal objects of interest.

Definition 2.3 (Original Stopped Martingale Problem). Fix $x_{\circ} \in \overline{\mathbf{S}}$. For each $\varepsilon>0$, let $\mathbb{P}^{\varepsilon} \in \mathscr{P}(C([0, \infty) ; \overline{\mathbf{S}}))$ be a solution to the stopped martingale problem with generator $\mathscr{L}^{\varepsilon}$ whose domain contains $C^{2}\left(\mathbb{R}^{2}\right)$ (as a dense subset), initial condition $\delta_{x_{\circ}}$, and stopping time $\mathfrak{e}$. Let $\mathbb{E}^{\varepsilon}$ be the corresponding expectation operator. This means the following. Firstly, that $\mathbb{P}^{\varepsilon}\left\{X_{0}=x_{0}\right\}=1$. Secondly, that if we fix $f \in C^{2}\left(\mathbb{R}^{2}\right), 0 \leq r_{1}<r_{2}<\cdots<r_{n} \leq s<t$ and $\left\{\varphi_{j} ; j=1,2 \ldots n\right\} \subset C_{b}\left(\mathbb{R}^{2}\right)$, then

$$
\mathbb{E}^{\varepsilon}\left[\left\{f\left(X_{t \wedge \mathfrak{e}}\right)-f\left(X_{s \wedge \mathfrak{e}}\right)-\int_{s \wedge \mathfrak{e}}^{t \wedge \mathfrak{e}}\left(\mathscr{L}^{\varepsilon} f\right)\left(X_{u}\right) d u\right\} \prod_{j=1}^{n} \varphi_{j}\left(X_{r_{j}}\right)\right]=0 .
$$

More or less, under $\mathbb{P}^{\varepsilon}, X=\left(x^{1}, x^{2}\right)$ satisfies the stochastic differential equation (1)

$$
\begin{aligned}
d x_{t}^{1} & =\frac{1}{\varepsilon^{2}} \frac{\partial H}{\partial x_{2}}\left(x_{t}^{1}, x_{t}^{2}\right) d t+b_{1}\left(x_{t}^{1}, x_{t}^{2}\right) d t+\sum_{i \in\{1,2\}} a_{1, i}\left(x_{t}^{1}, x_{t}^{2}\right) d W_{t}^{i}, \\
d x_{t}^{2} & =-\frac{1}{\varepsilon^{2}} \frac{\partial H}{\partial x_{1}}\left(x_{t}^{1}, x_{t}^{2}\right) d t+b_{2}\left(x_{t}^{1}, x_{t}^{2}\right) d t+\sum_{i \in\{1,2\}} a_{2, i}\left(x_{t}^{1}, x_{t}^{2}\right) d W_{t}^{i}, \quad t \geq 0, \\
\left(x_{0}^{1}, x_{0}^{2}\right) & =x_{\circ}
\end{aligned}
$$

up until time $\mathfrak{e}$, where $W^{1}$ and $W^{2}$ are some independent Wiener processes. We note that if in addition to Assumption 2.1, $\bar{\nabla} H$ is assumed to be Lipschitz and of linear growth, then standard calculations ensure that (1) has a solution for all time. We have a canonical $H$ in mind, however (see Remark 2.5), for which $\bar{\nabla} H$ is not globally Lipschitz, so we feel ethically compelled to content ourselves with a localized theory (which allows for sufficiently smooth coefficients which may have bad growth at infinity).

We are interested in the behavior of $\mathbb{P}^{\varepsilon}$ as $\varepsilon$ tends to zero. Since $(\bar{\nabla} H) H \equiv 0$, the variation of $H(X)$ under $\mathbb{P}^{\varepsilon}$ is of order 1 as $\varepsilon$ tends to zero (i.e., $H\left(X_{t}\right)$ is the slow variable). Classical stochastic averaging theory, which requires that $H$ have a single nondegenerate critical point, suggests that we look at the $\mathbb{P}^{\varepsilon}$ law of $H\left(X_{t}\right)$ and try to show that it converges to a Markov process. Our interest here is when the set of critical points actually has an interior. We will assume that $H$ has a certain general representation.

Assumption 2.4 (Representation of Hamiltonian). Assume that $\mathfrak{D} \in C^{\infty}\left(\mathbb{R}^{2}\right)$ is nonsingular on $\left\{x \in \mathbb{R}^{2}: \mathfrak{D}(x) \geq 0\right\}$ (i.e., $d \mathfrak{D}(x) \neq 0$ whenever $\mathfrak{D}(x) \geq 0$ ). We assume that

$$
\mathfrak{z} \stackrel{\text { def }}{=}\left\{x \in \mathbb{R}^{2}: \mathfrak{D}(x) \leq 0\right\}
$$




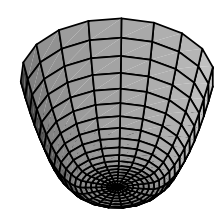

View from Below

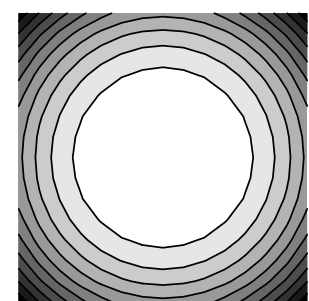

Contour Plot

Figure 1. Potential Energy Function for Canonical Hamiltonian

is a diffeomorphism of the unit disk, $\lim _{|x| \rightarrow \infty} \mathfrak{D}(x)=\infty$, and that

$$
H(x) \stackrel{\text { def }}{=} \max \{0, \mathfrak{D}(x)\}^{n}, \quad x \in \mathbb{R}^{2},
$$

for some $n>2$.

Essentially, this means that the function

$$
\mathfrak{D}(x)=(H(x))^{1 / n}, \quad x \notin \mathfrak{z},
$$

has a smooth extension inside $\mathfrak{z}$. Also, Assumption 2.4 implies several other things. First, that

$$
\left\{x \in \mathbb{R}^{2}: d H(x)=0\right\}=\left\{x \in \mathbb{R}^{2}: H(x)=0\right\}=\mathfrak{z} .
$$

Second, that for each $h>0, H^{-1}(h)$ is a connected smooth curve with finite $\mathscr{H}^{1}$ measure (where $\mathscr{H}^{1}$ is standard 1-dimensional Hausdorff measure). Thirdly, the above assumptions imply that

$$
\bar{\nabla} H=n(\max \{0, \mathfrak{D}(x)\})^{n-1} \bar{\nabla} \mathfrak{D}
$$

and from this we see that $\bar{\nabla} H$ is locally Lipschitz.

One should keep the following simple example in mind.

Remark 2.5 (Canonical Example). The simplest sort of Hamiltonian with a "flat" area is

$$
H_{\text {can }}(x)=\left\{\left(\|x\|_{\mathbb{R}^{2}}-1\right)^{+}\right\}^{n}, \quad x \in \mathbb{R}^{2},
$$

where $n>2$ (and where $\|\cdot\|_{\mathbb{R}^{2}}$ is the standard Euclidean distance on $\mathbb{R}^{2}$ ). Here we can take $\mathfrak{D} \in C^{\infty}\left(\mathbb{R}^{2}\right)$ so that $\mathfrak{D}(x)=\|x\|_{\mathbb{R}^{2}}-1$ for $\|x\|_{\mathbb{R}^{2}} \geq 1$. It helps to keep this example, which is pictured in Figure 1, in mind.

The point of classical stochastic averaging is an asymptotic separation of scales; under $\mathbb{P}^{\varepsilon}$, we can hope to find some closed dynamics for the slow variable by averaging over the invariant measure of the fast variable. Consider the flow

$$
\begin{aligned}
& \dot{\mathfrak{g}}_{t}(x)=\bar{\nabla} H\left(\mathfrak{g}_{t}(x)\right), \quad t \in \mathbb{R}, x \in \mathbb{R}^{2} . \\
& \mathfrak{g}_{0}(x)=x,
\end{aligned}
$$

We want to use $\mathfrak{g}$ to generate an equivalence relation on the original state space $\overline{\mathbf{S}}$. If $x \in \overline{\mathbf{S}} \backslash \mathfrak{z}$, then $\left\{\mathfrak{g}_{t}(x) ; t \in \mathbb{R}\right\}$ is the level set $H^{-1}(H(x))=\mathfrak{D}^{-1}(\mathfrak{D}(x))$, and if $x \in \mathfrak{z}$, then $\mathfrak{g}_{t}(x)=x$ for all $t \in \mathbb{R}$. If we were simply to take the quotient of $\overline{\mathbf{S}}$ by the standard group action, then the quotient space would be the two-dimensional 
disk $\mathfrak{z}$ and a collection of orbits which could be parametrized by points of $(0, \bar{H}]$. In particular, each element of $\partial \mathfrak{z}$ would be its own equivalence class. Intuitively, however, we also need to identify $\partial \mathfrak{z}$ as the limit of orbits in $\mathbb{R}^{2} \backslash \mathfrak{z}$. This stems from the fact that if we start the SDE (I) on a point of $\partial \mathfrak{z}$, the diffusion could kick the trajectory into $\overline{\mathbf{S}} \backslash \mathfrak{z}$, where the fast drift would quickly take it to a neighborhood of any other point of $\partial \mathfrak{z}$; i.e., we need to identify all elements of $\partial \mathfrak{z}$. This naturally occurs in chain equivalence. We shall follow the notation of [22]. We say that there is a $\delta$-chain of length $T>0$ from $x \in \mathbb{R}^{2}$ to $y \in \mathbb{R}^{2}$ if there is a sequence $\left(z_{j} ; j=1,2 \ldots n\right)$ of points in $\mathbb{R}^{2}$ and a sequence $0=t_{0}<t_{1}<\cdots<t_{n}=T$ of times such that $z_{0}=x$ and $z_{n}=y$ and such that $\left\|\mathfrak{g}_{t_{j}-t_{j-1}}\left(z_{j-1}\right)-z_{j}\right\|<\delta$ for all $1 \leq j \leq n$. We note that for each point $x \in \mathbb{R}^{2}$ and each $T>0$, there is a $\delta$-chain of length $T^{\prime}>T$ from itself to itself for each $\delta>0$ (the orbits of $\mathfrak{g}$ are periodic on $\overline{\mathbf{S}} \backslash \mathfrak{z}$ and $\mathfrak{z}$ consists of fixed points of $\mathfrak{g}$ ); thus the chain recurrent set of (3) is all of $\mathbb{R}^{2}$. We then define an equivalence relation $\sim$ (chain equivalence) on $\mathbb{R}^{2}$ by saying that $x \sim y$ if for each $T>0$ and each $\delta>0$ there is a $\delta$-chain of length $T^{\prime}>T$ from $x$ to $y$ and a $\delta$-chain of length $T^{\prime}>T$ from $y$ to $x$. This is the "correct" general equivalence relation in that two points are equivalent if a combination of a small diffusive perturbation and the fast drift takes one point to the other and back again. Define

$$
\mathfrak{M} \stackrel{\text { def }}{=} \overline{\mathbf{S}} / \sim
$$

and endow $\mathfrak{M}$ with the quotient topology defined by $\sim$. If $x \in \overline{\mathbf{S}}$, we let $[x] \stackrel{\text { def }}{=}$ $\{y \in \overline{\mathbf{S}}: y \sim x\}$ be the equivalence class of $x$ (the chain components of $\mathbb{R}^{2}$ ) and we define $\pi(x) \stackrel{\text { def }}{=}[x]$. In line with our above comments, we note that $\pi(x)=\{x\}$ if $x \in \mathfrak{z}^{\circ}, \pi(x)=H^{-1}(H(x))=\mathfrak{D}^{-1}(\mathfrak{D}(x))$ if $x \in \overline{\mathbf{S}} \backslash \mathfrak{z}$, and $\pi(x)=\partial \mathfrak{z}$ if $x \in \partial \mathfrak{z}$ (i.e., all points in $\partial \mathfrak{z}$ are equivalent). Thus

$$
\mathfrak{M}=\mathfrak{z}^{\circ} \cup \star \cup \Gamma \cup \circledast
$$

where

$$
\begin{gathered}
\mathfrak{z}^{\circ}=\bigcup_{x \in \mathfrak{z}^{\circ}}[x], \quad \star=\partial \mathfrak{z}=\bigcup_{x \in \partial \mathfrak{z}}[x], \\
\Gamma=\bigcup_{x \in \mathbf{S} \backslash \mathfrak{z}}[x], \quad \text { and } \quad \circledast=\bigcup_{x \in \partial \mathbf{S}}[x] .
\end{gathered}
$$

It is easy to see that $\mathfrak{z}^{\circ}$ is a two-dimensional open $C^{\infty}$ manifold, $\Gamma$ is a onedimensional open $C^{\infty}$ manifold, the point $\star$ is the limit of points in both $\mathfrak{z}^{\circ}$ and $\Gamma$, and the point $\circledast$ is the limit of points in $\Gamma$. This makes $\mathfrak{M}$ into a stratified space 12] if we enforce the ordering $\star \prec \mathfrak{z}^{\circ}, \star \prec \Gamma$, and $\circledast \prec \Gamma$. We also note that there is a homeomorphism between $\mathfrak{M}$ and a "whiskered sphere" in $\mathbb{R}^{3}$. First, let $\mathbf{i}_{2}$ be a diffeomorphism from $\mathfrak{z}$ to the unit disk $D \stackrel{\text { def }}{=}\left\{x \in \mathbb{R}^{2}:\|x\|_{\mathbb{R}^{2}} \leq 1\right\}$ (the existence of $\mathbf{i}_{2}$ is ensured by Assumption 2.4). Next let $\mathbf{i}_{1}: D \rightarrow S^{2}$ be defined by stereographic projection such that $\mathbf{i}_{1}(\partial D)=(0,0,1)$. We then define

$$
\mathbf{i}(x) \stackrel{\text { def }}{=} \begin{cases}\left(\mathbf{i}_{1} \circ \mathbf{i}_{2}\right)(x) & \text { if } x \in \mathfrak{z}^{\circ}, \\ (0,0,1) & \text { if } x=\star, \\ (0,0, H(x)+1) & \text { if } x \in \overline{\mathbf{S}} \backslash \mathfrak{z} .\end{cases}
$$

The image of $\mathbf{i}$ is the whiskered sphere $S^{2} \cup(\{0\} \times\{0\} \times[1, \bar{H}])$; see Figure 2 Since 


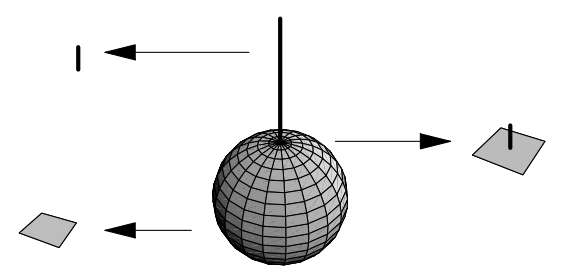

Figure 2. The Lollipop

i is a homeomorphism into $\mathbb{R}^{3}$, we can define the metric

$$
\rho(\pi(x), \pi(y)) \stackrel{\text { def }}{=}\|(\mathbf{i} \circ \pi)(x)-(\mathbf{i} \circ \pi)(y)\|_{\mathbb{R}^{3}}, \quad x, y \in \overline{\mathbf{S}},
$$

where $\|\cdot\|_{\mathbb{R}^{3}}$ is the standard Euclidean norm on $\mathbb{R}^{3}$. It is now easy to see that $\mathfrak{M}$ is in fact Polish.

Remark 2.6. From Figure 2 we see that $\mathfrak{M}$ is the union of a ball and a line. On the surface of the ball, the model space for $\mathfrak{M}$ is the plane (i.e., the surface of the ball is 2-dimensional), and on the line, the model space for $\mathfrak{M}$ is the (one-dimensional) line. The model space for $\mathfrak{M}$ at $\star$ is the union of a line and a plane.

Returning to averaging, we want to average the dynamics of the slow variables to get an effective $\mathfrak{M}$-valued process. We define an averaging operator $\mathbf{A}$ to do this.

Definition 2.7 (Averaging Operator). First, define a pre-averaging operator $\mathbf{A}^{\circ}$. The domain of $\mathbf{A}^{\circ}$ is the set of all real-valued mappings $\varphi$ whose domain contains $\mathbf{S} \backslash \partial \mathfrak{z}$ and such that $\left.\varphi\right|_{\mathbf{S} \backslash \partial \mathfrak{z}} \in B(\mathbf{S} \backslash \partial \mathfrak{z})$ (note that $\mathbf{S} \backslash \partial \mathfrak{z}=\mathfrak{z}^{\circ} \cup(\mathbf{S} \backslash \mathfrak{z})=\pi^{-1}\left(\mathfrak{z}^{\circ} \cup \Gamma\right)$ ). For such a $\varphi$, define $\mathbf{A}^{\circ} \varphi \in B\left(\mathfrak{z}^{\circ} \cup \Gamma\right)$ by the formula

$$
\left(\mathbf{A}^{\circ} \varphi\right)([x])= \begin{cases}\varphi(x) & \text { if } x \in \mathfrak{z}^{\circ}, \\ \frac{\int_{y \in[x]} \varphi(y)\|\bar{\nabla} H(y)\|_{\mathbb{R}^{2}}^{-1} \mathscr{H}^{1}(d y)}{\int_{y \in[x]}\|\bar{\nabla} H(y)\|_{\mathbb{R}^{2}}^{-1} \mathscr{H}^{1}(d y)} & \text { if } x \in \mathbf{S} \backslash \mathfrak{z}\end{cases}
$$

(where $\|\cdot\|_{T \mathbb{R}^{2}}$ is the standard metric on $T \mathbb{R}^{2}$ ). We say that $\varphi \in \mathscr{D}_{\mathbf{A}}$ if $\varphi$ is in the domain of $\mathbf{A}^{\circ}$ and the limits

$$
\lim _{\substack{[x] \rightarrow \star \\[x] \in \mathfrak{z}^{\circ} \cup \Gamma}}\left(\mathbf{A}^{\circ} \varphi\right)([x]) \quad \text { and } \quad \lim _{\substack{[x] \rightarrow \circledast \\[x] \in \mathfrak{z}^{\circ} \cup \Gamma}}\left(\mathbf{A}^{\circ} \varphi\right)([x])
$$

exist; if $\varphi \in \mathscr{D}_{\mathbf{A}}$, we define $(\mathbf{A} \varphi)([x]) \stackrel{\text { def }}{=} \lim _{[y] \rightarrow[x]}\left(\mathbf{A}^{\circ} \varphi\right)([y])$ for all $[x] \in \mathfrak{M}$.

If $\varphi \in C(\overline{\mathbf{S}}) \cap \mathscr{D}_{\mathbf{A}}$, then

$$
(\mathbf{A} \varphi)([x])=\lim _{T \rightarrow \infty} \frac{1}{T} \int_{0}^{T} \varphi\left(\mathfrak{g}_{s}(x)\right) d s
$$

for all $x \in \mathfrak{M}$.

Our goal is to show that the $\mathbb{P}^{\varepsilon}$-law of

$$
Y_{t} \stackrel{\text { def }}{=}\left[X_{t \wedge \mathfrak{e}}\right], \quad t \geq 0,
$$

converges to that of an $\mathfrak{M}$-valued Markov process as $\varepsilon$ tends to zero and to identify the generator of the limiting law. To be even more precise, for each $x \in \overline{\mathbf{S}}$ and 
$\varepsilon>0$, define the probability measure

$$
\mathbb{P}^{\varepsilon, \dagger}(A) \stackrel{\text { def }}{=} \mathbb{P}^{\varepsilon}\{Y \in A\} ; \quad A \in \mathscr{B}(C([0, \infty), \overline{\mathbf{S}}))
$$

we want to understand what the asymptotics of the $\mathbb{P}^{\varepsilon, \dagger}$ 's are as $\varepsilon$ tends to zero.

Let's define the usual averaged generator. Fix $f \in C^{2}\left(\mathfrak{z}^{\circ} \cup \Gamma\right)$; then $f \circ \pi$ is $C^{2}$ on $\mathbf{S} \backslash \partial \mathfrak{z}$, so $\mathscr{L}(f \circ \pi)$ is well-defined on $\mathbf{S} \backslash \partial \mathfrak{z}$. If $\mathscr{L}(f \circ \pi) \in \mathscr{D} \mathbf{A}$, we set

$$
\left(\mathscr{L}_{\text {ave }} f\right)([x]) \stackrel{\text { def }}{=}(\mathbf{A}(\mathscr{L}(f \circ \pi)))([x]), \quad[x] \in \mathfrak{M} .
$$

We then expect that the limiting dynamics of $Y$ will be given by the generator $\mathscr{L}_{\text {ave }}$ whose domain should be some subset of such $f$ such that in addition

$$
\lim _{\substack{[x] \rightarrow \circledast) \\[x] \in \mathfrak{z}^{\circ} \cup \Gamma}}\left(\mathscr{L}_{\text {ave }} f\right)([x])=0 .
$$

This encompasses what we expect when the process $X$ is not at $\star ;$ the $\mathbb{P}^{\varepsilon}$ law of $\left\{\left[X_{t}\right] ; t \geq 0\right\}$ tends to the solution of the martingale problem with the standard averaged generator up to the time when $X$ exits $\mathbf{S} \backslash \mathfrak{z}$, and where it is killed upon hitting $\circledast$. On the other hand, the $\mathbb{P}^{\varepsilon}$ law of $\left\{\left[X_{t}\right] ; t \geq 0\right\}$ tends to the solution of the martingale problem for the original generator $\mathscr{L}$ up to the time when $X$ exits $\mathfrak{z}^{\circ}$ (inside $\mathfrak{z}^{\circ}$, there is no fast motion).

We need to glue these generators together to specify what happens to $Y$ when it hits $\star$. The effect of glueing these two generators together is to enforce requirements on the domain of the overall generator at $\star$; see Remark 2.12 For $f \in C^{1}\left(\mathfrak{z}^{\circ}\right)$, we define an "inner" glueing operator

$$
\mathscr{G}_{\text {inner }} f \stackrel{\text { def }}{=} \lim _{\substack{[x] \rightarrow \star \\[x] \in \mathfrak{z}^{\circ}}} \frac{\int_{y \in[x]}\langle d f, d \mathfrak{D}\rangle(y)\|\bar{\nabla} \mathfrak{D}(y)\|_{T \mathbb{R}^{2}}^{-1} \mathscr{H}^{1}(d y)}{\int_{y \in[x]}\|\bar{\nabla} \mathfrak{D}(y)\|_{T \mathbb{R}^{2}}^{-1} \mathscr{H}^{1}(d y)}
$$

if this limit exists. We next lift $\mathfrak{D}$ to $\Gamma$; define $\tilde{\mathfrak{D}} \in C^{2}(\Gamma)$ by the formula

$$
\mathfrak{D}(x)=\tilde{\mathfrak{D}}([x]), \quad[x] \in \Gamma .
$$

Since $\mathscr{L}_{\text {ave }}$ is a nondegenerate elliptic operator on $C^{2}(\Gamma)$ we can consequently define the nonnegative bilinear form $\langle\cdot, \cdot\rangle_{\text {ave }}$ on $T^{*} \Gamma$ by

$$
\begin{aligned}
\langle d f, d g\rangle_{\text {ave }}([x])= & \left(\mathscr{L}_{\text {ave }}(f g)\right)([x])-f([x])\left(\mathscr{L}_{\text {ave }} g\right)([x]) \\
& -g([x])\left(\mathscr{L}_{\text {ave }} f\right)([x]), \quad[x] \in \Gamma,
\end{aligned}
$$

for all $f$ and $g$ in $C(\mathfrak{M})$ which are $C^{2}$ on $\mathfrak{z}^{\circ} \cup \Gamma$ and such that $\mathscr{L}(f \circ \pi), \mathscr{L}(g \circ \pi)$, and $\mathscr{L}((f g) \circ \pi)$ are all in $\mathscr{D}_{\mathbf{A}}$; in this case, $\langle d(f \circ \pi), d(g \circ \pi)\rangle \in \mathscr{D}_{\mathbf{A}}$, and

$$
\langle d f, d g\rangle_{\text {ave }}([x])=(\mathbf{A}\langle d(f \circ \pi), d(g \circ \pi)\rangle)([x])
$$

for all $[x] \in \Gamma$. For $f \in C^{1}(\Gamma)$, we define an "outer" glueing operator

$$
\mathscr{G}_{\text {outer }} f \stackrel{\text { def }}{=} \lim _{\substack{[x] \rightarrow \star \\[x] \in \Gamma}}\langle d f, d \tilde{\mathfrak{D}}\rangle_{\text {ave }}([x])
$$

if this limit exists. We will prove the following result in Section 9

Lemma 2.8. Fix $f \in C(\mathfrak{M})$ such that $f \in C^{2}\left(\mathfrak{z}^{\circ} \cup \Gamma\right)$ and $\mathscr{L}(f \circ \pi) \in \mathscr{D}_{\mathbf{A}}$. Then $\mathscr{G}_{\text {inner }} f$ and $\mathscr{G}_{\text {outer }} f$ exist.

Proof. Lemmas 9.5 and 9.7 
Note that if $f \in C^{1}\left(\mathbb{R}^{2}\right)$ is such that $\mathscr{G}_{\text {inner }} f$ exists, then

$$
\mathscr{G}_{\text {inner }} f=\lim _{\substack{[x] \rightarrow \star \\[x] \in \mathfrak{z}^{\circ}}}\left(\mathbf{A}^{\circ}\langle d f, d \mathfrak{D}\rangle\right)([x])
$$

thus $\mathscr{G}_{\text {inner }}$ defines an "averaged" derivative from the inside of $\mathfrak{z}$. This will help us in Remark 2.13 to explain the glueing conditions.

Remark 2.9. Since we have a natural nondegenerate symbol $\langle\cdot, \cdot\rangle$ defined on the open manifold $\mathfrak{z}^{\circ}$ and a natural nondegenerate symbol $\langle\cdot, \cdot\rangle_{\text {ave }}$ defined on the open manifold $\Gamma$, each of these open manifolds has an intrinsic Riemannian metric; thus the ball and the line in Figure 2 are both Riemannian manifolds. Any attempt to extend this Riemannian metric from $\mathbf{i}$ to all of $\mathbb{R}^{3}$ (i.e., to create an isometric embedding in $\mathbb{R}^{3}$ ) would, however, generate a singularity. One can take two sequences of points in $\mathfrak{z}^{\circ}$ which converge to different points of $\partial \mathfrak{z}$. Although these two sequences must converge to the same point in the topology of $\mathfrak{M}$, they clearly cannot do so in the Riemannian metric on $\mathfrak{z}^{\circ}$.

Let's start to write down things for our main result.

Definition 2.10 (Limiting Domain and Generator). Define

$$
\begin{aligned}
& \mathscr{D}^{\dagger} \stackrel{\text { def }}{=}\left\{f \in C(\mathfrak{M}):\left.f\right|_{\mathfrak{z}^{\circ} \cup \Gamma}\right. \in C^{2}\left(\mathfrak{z}^{\circ} \cup \Gamma\right), \mathscr{L}(f \circ \pi) \in \mathscr{D}_{\mathbf{A}}, \\
&\left.\mathscr{G}_{\text {inner }} f=\mathscr{G}_{\text {outer }} f \text { and } \lim _{\substack{[x] \rightarrow \mathfrak{z}^{\circ} \cup \Gamma \\
[x] \in \mathcal{L}^{\circledast}}}\left(\mathscr{L}_{\text {ave }} f\right)([x])=0\right\} .
\end{aligned}
$$

For $f \in \mathscr{D}^{\dagger}$, we define

$$
\left(\mathscr{L}^{\dagger} f\right)([x]) \stackrel{\text { def }}{=} \lim _{\substack{[y] \rightarrow[x] \\[y] \in \mathfrak{z}^{\circ} \cup \Gamma}}\left(\mathscr{L}_{\text {ave }} f\right)([y])
$$

for all $[y] \in \mathfrak{M}$.

The requirement that $\mathscr{G}_{\text {inner }} f=\mathscr{G}_{\text {outer }} f$ is the glueing conditions. See Remark 2.13 for an intepretation of the glueing condition as a continuity law. Let's next make the usual setup on the event space $\Omega^{\dagger} \stackrel{\text { def }}{=} C([0, \infty) ; \mathfrak{M})$. Define the coordinate functions $X_{t}^{\dagger}(\omega) \stackrel{\text { def }}{=} \omega(t)$ for all $t \geq 0$ and all $\omega \in \Omega^{\dagger}$. For each $t \geq 0$, define $\mathscr{F}_{t}^{\dagger} \stackrel{\text { def }}{=} \sigma\left\{X_{s}^{\dagger} ; 0 \leq s \leq t\right\}$ and define a sigma-algebra on $\Omega^{\dagger}$ by $\mathscr{F}^{\dagger} \stackrel{\text { def }}{=} \bigvee_{t \geq 0} \mathscr{F}_{t}^{\dagger}$.

Theorem 2.11 (Main Result). The $\mathbb{P}^{\varepsilon, \dagger}$ 's tend to the unique solution $\mathbb{P}^{\dagger}$ of the martingale problem with generator $\mathscr{L}^{\dagger}$ with domain $\mathscr{D}^{\dagger}$ and with initial condition $\delta_{\pi\left(x_{\circ}\right)}$. The martingale problem defining $\mathbb{P}^{\dagger}$ means the following. Firstly that $\mathbb{P}^{\dagger}\left\{X_{0}^{\dagger}=\pi\left(x_{\circ}\right)\right\}=1$. Secondly, that if we fix $f \in \mathscr{D}^{\dagger}, 0 \leq r_{1}<r_{2}<\cdots<r_{n} \leq$ $s<t$, and $\left\{\varphi_{j}^{\dagger} ; j=1,2 \ldots n\right\} \subset C(\mathfrak{M})$, then

$$
\mathbb{E}^{\dagger}\left[\left\{f\left(X_{t}^{\dagger}\right)-f\left(X_{s}^{\dagger}\right)-\int_{s}^{t}\left(\mathscr{L}^{\dagger} f\right)\left(X_{u}^{\dagger}\right) d u\right\} \prod_{j=1}^{n} \varphi_{j}^{\dagger}\left(X_{r_{j}}^{\dagger}\right)\right]=0 .
$$

Our proof of this result is (hopefully) arranged in a natural and easily-readable way. First, we will prove that the $\mathbb{P}^{\varepsilon, \dagger}$ 's are tight (Proposition [3.1); thus, by Prohorov's theorem, there must be at least one cluster point of the $\mathbb{P}^{\varepsilon, \dagger}$ 's in the weak 
topology of $\mathscr{P}(C([0, \infty) ; \mathfrak{M}))$. Our next goal (Proposition 4.1) is to prove that any such cluster point satisfies the martingale problem with domain and generator given in Definition 2.10 This will take some work. First we need to transfer (in some approximate sense) test functions in $\mathscr{D}^{\dagger}$ back to elements of $C^{2}\left(\mathbb{R}^{2}\right)$ (which is a dense subset of the domain of $\mathscr{L}^{\varepsilon}$ ), the domain of the original martingale problem (from Definition 2.3). This is done in Section 4, where we also set up some approximate calculations in the original state space $\overline{\mathbf{S}}$. Then we prove in Section 5 a bound on the amount of time that $X$ spends near $\partial \mathfrak{z}$; this will be useful in showing that various error terms which appear near $\partial \mathfrak{z}$ are negligible. In Section 6 we prove some stochastic averaging results. These averaging calculations must be carefully done since we need to average right up to the boundary $\partial \mathfrak{z}$; the glueing operators $\mathscr{G}_{\text {inner }}$ and $\mathscr{G}_{\text {outer }}$ come from some averaged boundary-layer quantities (see Remark 2.13 below). In Section 7 we return to the martingale problem and show that (5) must indeed be true for any cluster point of the $\mathbb{P}^{\varepsilon, \dagger}$ 's. At last, in Section 8, we show (Proposition 8.1) that $\mathscr{L}^{\dagger}$ generates a strongly continuous positive contraction semigroup on $C(\mathfrak{M})$, so the probability measure satisfying (5) must be unique. By [5, Theorem 4.8.2], this gives us the proof of our main result.

Remark 2.12. One can make an analogy between the limiting process on $\mathfrak{M}$ and skew Brownian motion [21. The trajectories of skew Brownian motion behave like ordinary Brownian motion until they hit zero. Informally, when a trajectory of skew Brownian motion hits zero, a coin (possibly biased) is flipped to determine whether the next excursion is positive or negative. The generator of the limiting process here is similar in spirit to the generator of skew Brownian motion (see [21] Exercise VII.2.1.23]. One could informally interpret the dynamics of the limiting process in our problem as follows. When the trajectory hits $\star$, a coin is flipped (based on the glueing coefficients) to determine whether the next excursion will be in $\Gamma$ or $\mathfrak{z}^{\circ}$. If the excursion is in $\mathfrak{z}^{\circ}$, the law by which the trajectory enters $\mathfrak{z}^{\circ}$ should be some type of (appropriately weighted) uniform distribution over $\partial \mathfrak{z}$. See [7] for a rigorous treatment of this construction.

Before starting our main arguments, let's introduce some notational conventions. For any function $F \in C^{1}\left(\mathbb{R}^{2}\right)$, we define the vector field (or more accurately derivation) $\nabla F$ as

$$
\begin{aligned}
((\nabla F) \varphi)\left(x_{1}, x_{2}\right) \stackrel{\text { def }}{=} \frac{\partial F}{\partial x_{1}}\left(x_{1}, x_{2}\right) \frac{\partial \varphi}{\partial x_{1}}\left(x_{1}, x_{2}\right)+\frac{\partial F}{\partial x_{2}}\left(x_{1}, x_{2}\right) \frac{\partial \varphi}{\partial x_{2}}\left(x_{1}, x_{2}\right), & \\
\varphi & \in C^{\infty}\left(\mathbb{R}^{2}\right),\left(x_{1}, x_{2}\right) \in \mathbb{R}^{2}
\end{aligned}
$$

(i.e., $\nabla F$ is the standard gradient of $F$ ).

It will also be useful to use $\mathfrak{D}$ to map $\Gamma$ into $\mathbb{R}$ (even though $H$, not $\mathfrak{D}$ is our original Hamiltonian). Define

$$
\bar{D} \stackrel{\text { def }}{=} \bar{H}^{1 / n} \text {. }
$$

If $\varphi$ is a real-valued function whose domain contains $\Gamma$, we define $\varphi_{D}:(0, \bar{D}) \rightarrow \mathbb{R}$ by

$$
\varphi_{D}(\mathfrak{D}(x))=\varphi([x]), \quad x \in \mathbf{S} \backslash \mathfrak{z} .
$$

Secondly, let $\varkappa \in(0, \bar{D})$ be such that $d \mathfrak{D}(x) \neq 0$ for all $x \in \mathbf{S}$ such that $|\mathfrak{D}(x)| \leq \varkappa$ (the existence of such a $\varkappa$ is ensured by continuity and Assumption 2.4). Define 
also

$$
\mathcal{N} \stackrel{\text { def }}{=}\{x \in \mathbf{S}:|\mathfrak{D}(x)|<\varkappa\}
$$

Let's also define

$$
\sigma^{2}([x]) \stackrel{\text { def }}{=}\left(\mathbf{A}^{\circ}\langle d \mathfrak{D}, d \mathfrak{D}\rangle\right)([x]) \quad \text { and } \quad b([x]) \stackrel{\text { def }}{=}\left(\mathbf{A}^{\circ}(\mathscr{L} \mathfrak{D})\right)([x])
$$

for all $x \in \overline{\mathbf{S}} \backslash \mathfrak{z}$. In light of our hypotheses (i.e., Assumption 2.4 ), we have that both $\sigma_{D}^{2}(0) \stackrel{\text { def }}{=} \lim _{d \searrow 0} \sigma_{D}^{2}(d)$ and $b_{D}(0) \stackrel{\text { def }}{=} \lim _{d \searrow 0} b_{D}(d)$ exist and $\sigma_{D}^{2}(0)>0$. Finally, define the second-order differential operator $\mathscr{L}_{D}$ on $C^{2}((0, \bar{D}))$ as

$$
\left(\mathscr{L}_{D} \varphi\right)(d) \stackrel{\text { def }}{=} \frac{1}{2} \sigma_{D}^{2}(d) \ddot{\varphi}(d)+b_{D}(d) \dot{\varphi}(d), \quad d \in(0, \bar{D}), \varphi \in C^{2}((0, \bar{D})),
$$

then $\left(\mathscr{L}_{\text {ave }} \varphi\right)([x])=\left(\mathscr{L}_{D} \varphi_{D}\right)(\mathfrak{D}(x))$ for $x \in \mathbf{S} \backslash \mathfrak{z}$ and $\varphi \in C^{2}\left(\mathfrak{z}^{\circ} \cup \Gamma\right)$ such that $\mathscr{L}(\varphi \circ \pi) \in \mathscr{D}_{\mathbf{A}}$.

Using this notation, we can understand the glueing condition a bit better.

Remark 2.13. Essentially, the glueing condition can be thought of as a continuity equation and a conservation equation. Assume for the sake of argument that there is a $p_{\mathfrak{z}} \in C^{\infty}((0, \infty) \times \mathfrak{z})$, a $p_{D} \in C^{\infty}((0, \infty) \times[0, \bar{D}])$, and a $p_{\circledast}(t) \in C^{\infty}((0, \infty))$ such that

$$
\mathbb{E}^{\dagger}\left[f\left(X_{t}^{\dagger}\right)\right]=\int_{x \in \mathfrak{z}^{\circ}} p_{\mathfrak{z}}(t, x) f(x) d x+\int_{x=0}^{\bar{D}} p_{D}(t, x) f_{D}(x) d x+p_{\circledast}(t) f_{D}(\bar{D})
$$

for all $t>0$ and all $f \in \mathscr{D}^{\dagger}$, where $f_{D}(\bar{D}) \stackrel{\text { def }}{=} \lim _{d \nearrow \bar{D}} f_{D}(d)$ (since $\langle\cdot, \cdot\rangle$ is nondegenerate, $X$ spends zero time at $\partial \mathfrak{z}$, and consequently $X^{\dagger}$ must spend zero time at $\star$, so we shouldn't need a term at $\star$ on the right-hand side of this representation; on the other hand, since $X$ is killed at $\partial \mathbf{S}$, we do need a Dirac mass at $\circledast)$. Assuming enough smoothness,

$$
\begin{aligned}
& \int_{x \in \mathfrak{z}^{\circ}} \frac{\partial p_{\mathfrak{z}}}{\partial t}(t, x) f(x) d x+\int_{x=0}^{\bar{D}} \frac{\partial p_{D}}{\partial t}(t, x) f_{D}(x) d x+\dot{p}_{\circledast}(t) f_{D}(\bar{D}) \\
& =\int_{x \in \mathfrak{z}^{\circ}} p_{\mathfrak{z}}(t, x)(\mathscr{L} f)(x) d x+\int_{x=0}^{\bar{D}} p_{D}(t, x)\left(\mathscr{L}_{D} f_{D}\right)(x) d x+p_{\circledast}(t)\left(\mathscr{L}_{D} f_{D}\right)(\bar{D}) .
\end{aligned}
$$

Since the definition of $\mathscr{D}^{\dagger}$ ensures that $\left(\mathscr{L}_{D} f_{D}\right)(\bar{D})=0$, the last term is identically zero.

By taking the test function $f$ to have support in $\mathfrak{z}^{\circ}$ or $\Gamma$, we get that

$$
\begin{gathered}
\frac{\partial p_{\mathfrak{z}}}{\partial t}(t, x)=\left(\mathscr{L}^{\operatorname{adj}_{\mathbb{R}^{2}} p_{\mathfrak{z}}}\right)(t, x), \quad t>0, x \in \mathfrak{z}^{\circ}, \\
\frac{\partial p_{D}}{\partial t}(t, x)=\left(\mathscr{L}_{D}^{\operatorname{adj}_{\mathbb{R}}} p_{D}\right)(t, x), \quad t>0, x \in(0, \bar{D}),
\end{gathered}
$$

where $\mathscr{L}^{\text {adj }_{\mathbb{R}^{2}}}$ and $\mathscr{L}_{D}^{\text {adj }}$ are respectively the adjoints of $\mathscr{L}$ and $\mathscr{L}_{D}$ with respect to $\mathbb{R}^{2}$ and $\mathbb{R}$. This is the standard Chapman-Kolmogorov equation. Let's write these PDE's in more physically meaningful notation. Convert $\mathscr{L}$ and $\mathscr{L}_{D}$ to Hörmanderform operators; i.e., $\mathscr{L}=\frac{1}{2} \sum_{i=1}^{2} X_{i}^{2}+X_{0}$ and $\mathscr{L}_{D}=\frac{1}{2} Y_{1}^{2}+Y_{0}$, where the $X_{i}$ 's are vector fields on $\mathbb{R}^{2}$ and the $Y_{i}$ 's are vector fields on $\mathbb{R}$. Let $\operatorname{div}_{\mathbb{R}^{2}}$ and $\operatorname{div}_{\mathbb{R}}$ be respectively the standard divergence operator on vector fields on $\mathbb{R}^{2}$ and $\mathbb{R}$ 
(i.e., $\operatorname{div}_{\mathbb{R}^{2}}\left(\sum_{i=1}^{2} \alpha_{i} \frac{\partial}{\partial x_{i}}\right)=\sum_{i=1}^{2} \frac{\partial \alpha_{i}}{\partial x_{i}}$ and $\left.\operatorname{div}_{\mathbb{R}}\left(\alpha \frac{\partial}{\partial x}\right)=\frac{\partial \alpha}{\partial x}\right)$. Define the two flux vectors $\mathbf{J}_{\mathfrak{z}} \in T \mathbb{R}^{2}$ and $\mathbf{J}_{D} \in T \mathbb{R}$ by

$$
\begin{aligned}
& \mathbf{J}_{\mathfrak{z}}(t, x) \stackrel{\text { def }}{=}-\frac{1}{2} \sum_{i=1}^{2}\left(\operatorname{div}_{\mathbb{R}^{2}}\left(p_{\mathfrak{z}}(t, \cdot) X_{i}\right)\right)(x) X_{i}(x)+p_{\mathfrak{z}}(t, x) X_{0}(x), \quad x \in \mathfrak{z}, \\
& \mathbf{J}_{D}(t, x) \stackrel{\text { def }}{=}-\frac{1}{2}\left(\operatorname{div}_{\mathbb{R}}\left(p_{D}(t, \cdot) Y_{1}\right)\right)(x) Y_{1}(x)+p_{D}(t, x) Y_{0}(x), \quad x \in[0, \bar{D}],
\end{aligned}
$$

for all $t>0$; then the PDE's of (7) can be written as the conservation laws

$$
\begin{gathered}
\frac{\partial p_{\mathfrak{z}}}{\partial t}(t, x)=-\left(\operatorname{div}_{\mathbb{R}^{2}} \mathbf{J}_{\mathfrak{z}}(t, \cdot)\right)(x), \quad x \in \mathfrak{z}^{\circ}, \\
\frac{\partial p_{D}}{\partial t}(t, x)=-\left(\operatorname{div}_{\mathbb{R}^{2}} \mathbf{J}_{\mathfrak{z}}\right)(t, \cdot)(x), \quad x \in(0, \bar{D}),
\end{gathered}
$$

for all $t>0$.

We next consider more general test functions to get the boundary conditions for $p_{\mathfrak{z}}$ and $p_{D}$. Using the Gauss-Green theorem and (7), we find that

$$
\begin{aligned}
\dot{p}_{\circledast}(t) f_{D}(\bar{D})= & \frac{1}{2} \sum_{i=1}^{2} \int_{x \in \partial \mathfrak{z}} p_{\mathfrak{z}}(t, x)\left(\nu_{\mathfrak{z}}(x), X_{i}(x)\right)_{T \mathbb{R}^{2}}\left(X_{i} f_{\mathfrak{z}}\right)(x) \mathscr{H}^{1}(d x) \\
& +\int_{x \in \partial \mathfrak{z}} f_{\mathfrak{z}}(x)\left(\nu_{\mathfrak{z}}(x), \mathbf{J}_{\mathfrak{z}}(t, x)\right)_{T \mathbb{R}^{2}} \mathscr{H}^{1}(d x) \\
& +\frac{1}{2} p_{D}(t, \bar{D})\left(\frac{\partial}{\partial x}, Y_{1}(\bar{D})\right)_{T \mathbb{R}}\left(Y_{1} f_{D}\right)(\bar{D}) \\
& -\frac{1}{2} p_{D}(t, 0)\left(\frac{\partial}{\partial x}, Y_{1}(0)\right)_{T \mathbb{R}}\left(Y_{1} f_{D}\right)(0) \\
& +f_{D}(\bar{D})\left(\frac{\partial}{\partial x}, \mathbf{J}_{\mathfrak{z}}(t, \bar{D})\right)_{T \mathbb{R}}-f_{D}(0)\left(\frac{\partial}{\partial x}, \mathbf{J}_{\mathfrak{z}}(t, 0)\right)_{T \mathbb{R}}
\end{aligned}
$$

Here $(\cdot, \cdot)_{T \mathbb{R}}$ is the standard metric on $T \mathbb{R}$ and $\nu_{\mathfrak{z}}(x)=\frac{\nabla \mathfrak{D}(x)}{\|\nabla \mathfrak{D}(x)\|_{T \mathbb{R}^{2}}}$ is the outwardpointing normal to $\mathfrak{z}$ at $x \in \partial \mathfrak{z}$. Choosing now $f \in \mathscr{D}^{\dagger}$ which is constant in a neighborhood of $\star$ and $\circledast$ (it is easy to see that there are indeed such functions in $\mathscr{D}^{\dagger}$ ), we get the flux conditions

$$
\begin{gathered}
\dot{p}_{\circledast}(t)=\left(\frac{\partial}{\partial x}, \mathbf{J}_{\mathfrak{z}}(t, \bar{D})\right)_{T \mathbb{R}}, \\
\int_{x \in \partial \mathfrak{z}}\left(\nu_{\mathfrak{z}}(x), \mathbf{J}_{\mathfrak{z}}(t, x)\right)_{T \mathbb{R}^{2}} \mathscr{H}^{1}(d x)=\left(\frac{\partial}{\partial x}, \mathbf{J}_{\mathfrak{z}}(t, 0)\right)_{T \mathbb{R}}
\end{gathered}
$$

for all $t>0$ (remember that $\left.f_{\mathfrak{z}}\right|_{\partial \mathfrak{z}} \equiv f_{D}(0)$ ). The first equation is the natural one stating that the flux leaving $(0, \bar{D})$ through $\bar{D}$ enters $\circledast$. The second one states that the flux entering $(0, \bar{D})$ through 0 is equal to the total (i.e., integrated) flux leaving $\mathfrak{z}$ (through $\partial \mathfrak{z})$. Note next that

$$
\begin{gathered}
\sum_{i=1}^{2}\left(\nu_{\mathfrak{z}}(x), X_{i}(x)\right)_{T \mathbb{R}^{2}}\left(X_{i} f_{\mathfrak{z}}\right)(x)=\frac{\left\langle d f_{\mathfrak{z}}, d \mathfrak{D}\right\rangle(x)}{\|\nabla \mathfrak{D}(x)\|_{T \mathbb{R}^{2}}}, \quad x \in \partial \mathfrak{z}, \\
\left(\frac{\partial}{\partial x}, Y_{1}(x)\right)_{T \mathbb{R}}\left(Y_{1} f_{D}\right)(x)=\dot{f}_{D}(x) \sigma_{D}^{2}(x), \quad x \in\{0, \bar{D}\} .
\end{gathered}
$$


We also expect that $p_{\mathfrak{z}}$ is constant on $\partial \mathfrak{z}$ (since $\partial \mathfrak{z}$ corresponds to the single point $\star$ in $\mathfrak{M}$ ). Using (9) in (8) and using the definition of $\mathscr{G}_{\text {inner }} f$, we get that

$$
\begin{gathered}
p_{D}(t, \bar{D})=0, \\
\left.p_{\mathfrak{z}}(t, \cdot)\right|_{\partial \mathfrak{z}} \int_{x \in \partial}\|\bar{\nabla} \mathfrak{D}(x)\|_{T \mathbb{R}^{2}}^{-1} \mathscr{H}^{1}(d x) \mathscr{G}_{\text {inner }} f=\dot{f}_{D}(0) \sigma_{D}^{2}(0) p_{D}(t, 0) .
\end{gathered}
$$

The first equation signifies simply that particles are killed at $\bar{D}$. The glueing condition and the explicit formula for $\mathscr{G}_{\text {outer }} f$ (see Lemma 9.5) imply that $\mathscr{G}_{\text {inner }} f=$ $\dot{f}_{D}(0) \sigma_{D}^{2}(0)$. Note also that the integral in the second equation is exactly $\mathfrak{T}^{D}(0)$ of (15); thus the second equation implies that

$$
\left.\mathfrak{T}^{D}(0) p_{\mathfrak{z}}(t, \cdot)\right|_{\partial \mathfrak{z}}=p_{D}(t, 0)
$$

for all $t>0$. Thus the likelihood of finding a particle in $\Gamma$ near $\star$ is the same as the likelihood of finding a particle in $\mathfrak{z}$ near $\partial \mathfrak{z}$ times the normalizing constant $\mathfrak{T}^{D}(0)$ (the asymptotically normalized orbit time of the fast motion).

As a final notational convention, we set up some definitions involving differentials of functions. If $S$ is some subset of some $\mathbb{R}^{N}$, we say that a real-valued mapping $\varphi$ whose domain contains $S$ is in $C^{k}(S)$ if all partial derivatives of $\varphi$ of order $k$ or less exist and are continuous at each point of the interior of $S$ and if the limits of all such partial derivatives exist at each point of $S \backslash S^{\circ}$. If $S^{\prime} \subset S$ and $\varphi \in C^{k}(S)$, we define $\|\varphi\|_{C^{k}\left(S^{\prime}\right)}$ as the supremum of $\left|D^{\alpha} \varphi(x)\right|$ over all multi-indices (see [6]) of order $k$ or less and all $x \in S^{\prime}$.

\section{Tightness}

We will prove the following result, which by Prohorov's theorem implies tightness of the $\mathbb{P}^{\varepsilon, \dagger}$ 's. Recall the metric $\rho$ of (4) on $\mathfrak{M}$.

Proposition 3.1. For each $\delta>0$ and $T>0$,

$$
\varlimsup_{\eta \rightarrow 0} \sup _{\varepsilon>0} \mathbb{P}^{\varepsilon}\left\{\sup _{\substack{0 \leq s<t \leq T \wedge \mathfrak{e} \\|s-t|<\eta}} \rho\left(Y_{s}, Y_{t}\right) \geq \delta\right\}=0 .
$$

Thus, the $\mathbb{P}^{\varepsilon, \dagger}$ 's are tight in the Prohorov topology on $\mathscr{P}(C([0, \infty) ; \mathfrak{M}))$.

We will prove this in a collection of bite-sized pieces. First, we realize that we can restrict our calculations to $s$ such that $Y_{s}$ is not too close to $\star$; if $Y$ has large variation, it can't all be too close to *.

Lemma 3.2. For each $\delta>0, T>0, \varepsilon>0$, and $\eta>0$,

$$
\mathbb{P}^{\varepsilon}\left\{\sup _{\substack{0 \leq s<t \leq T \wedge \mathfrak{e} \\|s-t|<\eta}} \rho\left(Y_{s}, Y_{t}\right) \geq \delta\right\} \leq \mathbb{P}^{\varepsilon}\left\{\sup _{\substack{0 \leq s<t \leq T \wedge \mathfrak{e} \\|s-t|<\eta \\ \rho\left(Y_{s}, \star\right) \geq \delta / 3}} \rho\left(Y_{s}, Y_{t}\right) \geq \delta / 6\right\} .
$$

We could actually prove this result with $\delta / 6$ on the left replaced by $\delta / 3$; it will be more directly useful, however, as we have stated it. 
Proof. Suppose that for a fixed $0 \leq s<t \leq T \wedge \mathfrak{e}$, we have that $|s-t|<\eta$ and $\rho\left(Y_{s}, Y_{t}\right) \geq \delta$. There are two possibilities, depending on $\rho\left(Y_{s}, \star\right)$. In the first case, $\rho\left(Y_{s}, \star\right) \geq \delta / 3$ and we have that $\rho\left(Y_{s}, Y_{t}\right) \geq \delta \geq \delta / 3$. Alternately, assume that $\rho\left(Y_{s}, \star\right)<\delta / 3$. We can use the triangle inequality to see that $\rho\left(Y_{t}, \star\right) \geq$ $\rho\left(Y_{t}, Y_{s}\right)-\rho\left(Y_{s}, \star\right)>\delta-\delta / 3=2 \delta / 3$; thus $\rho\left(Y_{s}, \star\right)<\delta / 3$ and $\rho\left(Y_{t}, \star\right)>2 \delta / 3$. Hence by continuity there is an $r \in(s, t)$ such that $\rho\left(Y_{r}, \star\right)=\delta / 3$. Another application of the triangle inequality shows that $\rho\left(Y_{r}, Y_{t}\right) \geq \rho\left(Y_{t}, \star\right)-\rho\left(Y_{r}, \star\right) \geq 2 \delta / 3-\delta / 3=\delta / 3$. This proves the result if we replace $\delta / 6$ on the right-hand side of (10) by $\delta / 3$. Since $\delta / 3>\delta / 6$, the stated result follows.

Fix now $\delta>0$ and $T>0$. Let's look at the coordinates of $\mathbf{i}\left(Y_{t}\right)$, or more precisely their variation. We want to use the restriction that $\rho\left(Y_{s}, \star\right) \geq \delta / 3$ stays away from the singularity of $\mathbf{i}$ at $\star$. Note that if $x$ and $y$ in $\mathfrak{M}$ are such that $\rho(x, \star) \geq \delta / 3$ and $\rho(x, y)=\delta / 6$, then by the triangle inequality,

$$
\|\mathbf{i}(y)-(0,0,1)\|_{\mathbb{R}^{3}}=\rho(y, \star) \geq \rho(x, \star)-\rho(x, y) \geq \delta / 6 .
$$

Fix a cutoff function $\varpi \in C_{c}^{\infty}\left(\mathbb{R}^{3}\right)$. We require that $\varpi(x)=1$ for all $x \in \mathbf{i}(\mathfrak{M})$ such that $\|x-(0,0,1)\|_{\mathbb{R}^{3}} \geq 2 \delta / 18$ and that $\varpi(x)=0$ if $\|x-(0,0,1)\|_{\mathbb{R}^{3}} \leq \delta / 18$. Let $\left\{\mathbf{e}_{1}, \mathbf{e}_{2}, \mathbf{e}_{3}\right\}$ be an orthonormal basis of $\mathbb{R}^{3}$, and define, for $i \in\{1,2,3\}$,

$$
f_{i}(x) \stackrel{\text { def }}{=}\left(\mathbf{i}(x), \mathbf{e}_{i}\right)_{\mathbb{R}^{3}} \varpi(\mathbf{i}(x)), \quad x \in \mathfrak{M},
$$

where $(\cdot, \cdot)_{\mathbb{R}^{3}}$ is the standard inner product in $\mathbb{R}^{3}$. Then $f_{i} \circ \pi$ is in the domain of $\mathscr{L}^{\varepsilon}$, and

$$
f_{i}\left(Y_{s}\right)=f_{i}\left(Y_{0}\right)+\int_{0}^{t} \mathscr{L}\left(f_{i} \circ \pi\right)\left(X_{s}\right) d s+M_{t}^{i}, \quad 0 \leq t \leq \mathfrak{e}
$$

where $M^{i}$ is a $\mathbb{P}^{\varepsilon}$-martingale with quadratic variation

$$
\left\langle M^{i}\right\rangle_{t} \stackrel{\text { def }}{=} \int_{0}^{t}\left\langle d\left(f_{i} \circ \pi\right)\left(X_{s}\right), d\left(f_{i} \circ \pi\right)\left(X_{s}\right)\right\rangle d s, \quad t \geq 0
$$

We have used here the fact that $\mathscr{L}^{\varepsilon}\left(f_{i} \circ \pi\right)=\mathscr{L}\left(f_{i} \circ \pi\right)$, which follows from the observation that $f_{i} \circ \pi(x)$ in fact depends only on $H(x)$ whenever $H(x) \geq 0$ and that $\bar{\nabla} H(x)=0$ if $x \in \mathfrak{z}$. For any $x$ and $y$ in $\mathfrak{M}$ such that $\rho(x, \star) \geq \delta / 3$ and $\rho(y, \star) \geq \delta / 6$

$$
\rho(x, y)=\left\{\sum_{i=1}^{3}\left(f_{i}(x)-f_{i}(y)\right)^{2}\right\}^{1 / 2}
$$

(since for such $x$ and $y$, the $f_{i}$ 's are just the coordinates of $\mathbf{i}(x)$ and $\mathbf{i}(y)$ ). We want to use this and (11) to study the variation of $t \mapsto Y_{t}$ as needed to bound the right-hand side of (10); i.e., as long as the trajectory stays outside a $\rho$-ball of radius $\delta / 6$. For this reason, let's decompose the $f_{i}(Y)$ 's into parts which vary when 
$\rho(Y, \star)>\delta / 6$ and parts which vary when $\rho(Y, \star) \leq \delta / 6$. Define

$$
\begin{aligned}
& A_{t}^{1, i} \stackrel{\text { def }}{=} \int_{0}^{t} \mathscr{L}\left(f_{i} \circ \pi\right)\left(X_{s}\right) \chi_{\left\{\rho\left(Y_{s}, \star\right)>\delta / 6\right\}} d s \\
& A_{t}^{2, i} \stackrel{\text { def }}{=} \int_{0}^{t} \mathscr{L}\left(f_{i} \circ \pi\right)\left(X_{s}\right) \chi_{\left\{\rho\left(Y_{s}, \star\right) \leq \delta / 6\right\}} d s \\
& M_{t}^{1, i} \stackrel{\text { def }}{=} \int_{0}^{t} \chi_{\left\{\rho\left(Y_{s}, \star\right)>\delta / 6\right\}} d M_{s}^{i}, \\
& M_{t}^{2, i} \stackrel{\text { def }}{=} \int_{0}^{t} \chi_{\left\{\rho\left(Y_{s}, \star\right) \leq \delta / 6\right\}} d M_{s}^{i},
\end{aligned}
$$

Then for any $s<t \leq \mathfrak{e}$,

$$
\begin{aligned}
f_{i}\left(Y_{t}\right)-f_{i}\left(Y_{s}\right)= & \left(A_{t}^{1, i}-A_{s}^{1, i}\right)+\left(A_{t}^{2, i}-A_{s}^{2, i}\right) \\
& +\left(M_{t}^{1, i}-M_{s}^{1, i}\right)+\left(M_{t}^{2, i}-M_{s}^{2, i}\right) .
\end{aligned}
$$

Our next step is

Lemma 3.3. For each $\delta>0, T>0, \varepsilon>0$ and $\eta>0$,

$$
\begin{aligned}
& \mathbb{P}^{\varepsilon}\left\{\sup _{\substack{0 \leq s<t \leq T \wedge \mathfrak{e} \\
|s-t|<\eta \\
\rho\left(Y_{s}, \star\right) \geq \delta / 3}} \rho\left(Y_{s}, Y_{t}\right) \geq \delta / 6\right\} \\
& \leq \sum_{i=1}^{3} \mathbb{P}^{\varepsilon}\left\{\sup _{\substack{0 \leq s<t \leq T \wedge \mathfrak{e} \\
|s-t|<\eta}}\left|A_{t}^{1, i}-A_{s}^{1, i}\right| \geq \frac{\delta}{12 \sqrt{3}}\right\} \\
& +\sum_{i=1}^{3} \mathbb{P}^{\varepsilon}\left\{\sup _{\substack{0 \leq s<t \leq T \wedge \mathfrak{e} \\
|s-t|<\eta}}\left|M_{t}^{1, i}-M_{s}^{1, i}\right| \geq \frac{\delta}{12 \sqrt{3}}\right\} .
\end{aligned}
$$

Proof. Assume that $0 \leq s<t \leq T \wedge \mathfrak{e}$ are such that $|s-t|<\eta, \rho\left(Y_{s}, \star\right) \geq \delta / 3$, and $\rho\left(Y_{s}, Y_{t}\right) \geq \delta / 6$. We want to find an $r \in(s, t]$ such that $\rho\left(Y_{r}, Y_{t}\right)=\delta / 6$, but $\rho\left(Y_{u}, \star\right)>\delta / 6$ for all $s<u<r$; then we can neglect the (possibly bad) $A^{2, i}$ 's and $M^{2, i}$ 's in (13). Indeed, since $\rho\left(Y_{s}, Y_{t}\right) \geq \delta / 6$, the time $r \stackrel{\text { def }}{=} \inf \{s>$ $\left.t: \rho\left(Y_{s}, Y_{r}\right)=\delta / 6\right\}$ is in $(s, t]$ and satisfies these requirements. By (12) and the calculation that $\rho\left(Y_{r}, \star\right) \geq \rho\left(Y_{s}, \star\right)-\rho\left(Y_{s}, Y_{r}\right) \geq \delta / 3-\delta / 6=\delta / 6$, we must have that $\left|f_{i}\left(Y_{s}\right)-f_{i}\left(Y_{r}\right)\right| \geq \frac{\delta}{6 \sqrt{3}}$ for some $i \in\{1,2,3\}$. We also note that for $s \leq u<r$, $\rho\left(Y_{u}, \star\right) \geq \rho\left(Y_{s}, \star\right)-\rho\left(Y_{u}, Y_{s}\right)>\delta / 3-\delta / 6=\delta / 6$, and hence $A_{r}^{2, i}-A_{s}^{2, i}=0$ and $M_{r}^{2, i}-M_{s}^{2, i}=0$. Thus by (13),

$$
\left.f_{i}\left(Y_{r}\right)-f_{i}\left(Y_{s}\right)=\left(A_{r \wedge \mathfrak{e}}^{1, i}-A_{s \wedge \mathfrak{e}}^{1, i}\right)+\left(M_{r \wedge \mathfrak{e}}^{1, i}-M_{s \wedge \mathfrak{e}}^{1, i}\right)\right)
$$

In a straightforward manner, this now gives the result.

We can now give the

Proof of Proposition 3.1. We note that $x \mapsto \mathscr{L}\left(f_{i} \circ \pi\right)(x) \chi_{\{\rho([x], \star)>\delta / 6\}}$ and $x \mapsto$ $\left\langle d\left(f_{i} \circ \pi\right)(x), d\left(f_{i} \circ \pi\right)(x)\right\rangle \chi_{\{\rho([x], \star)>\delta / 6\}}$ are bounded functions on $\mathbb{R}^{2}$. Standard calculations involving Lemmas $[3.2$ and 3.3 give the result. 


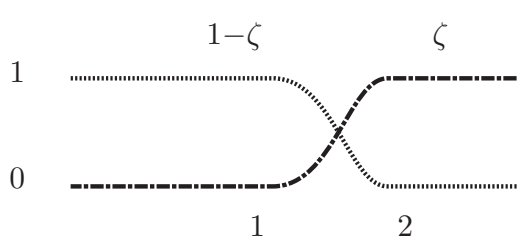

Figure 3. Cutoff Functions

\section{The Martingale Problem}

We now know that the $\mathbb{P}^{\varepsilon}$-laws of $\left\{Y_{t} ; t \geq 0\right\}$ are tight. We next need to show

Proposition 4.1. Let $\mathbb{P}^{\dagger} \in \mathscr{P}(C([0, \infty) ; \mathfrak{M}))$ be a cluster point of the $\mathbb{P}^{\varepsilon}, \dagger$ 's. Let $\mathbb{E}^{\dagger}$ be the associated expectation operator. Then (5) holds; in other words, any cluster point of the $\mathbb{P}^{\varepsilon, \dagger}$ 's satisfies the martingale problem associated with $\mathscr{L}^{\dagger}$.

Here we will gather together the ideas leading to this result, but many of the harder parts will be proved in the next several sections.

Our starting point is clearly Definition 2.10 so we should try to connect elements of $\mathscr{D}^{\dagger}$ back to elements of $C^{2}\left(\mathbb{R}^{2}\right)$. Fix an $f \in \mathscr{D}^{\dagger}$. Then

$$
(f \circ \pi)(x)=f(x) \chi_{\mathfrak{z}}(x)+f_{D}(\mathfrak{D}(x)) \chi_{\mathfrak{z}^{c}}(x), \quad x \in \overline{\mathbf{S}} .
$$

We want to use this representation to approximate $f \circ \pi$ by an element of $C^{2}\left(\mathbb{R}^{2}\right)$. We will do this in two steps. First, we want to smooth off the indicator functions by smooth functions. This will in turn force us to slightly extend the definitions of $f_{D}$ and $\left.f\right|_{\mathfrak{z}}$ (in fact, we will only use the extension of $\left.f\right|_{\mathfrak{z}}$; we will develop both extensions in the interest of symmetry). Central to these approximations will be some exponents.

Definition 4.2 (Smoothing exponents). Fix

$$
\nu=\frac{2}{n+1} \quad \text { and } \quad \nu^{\prime}<\frac{2}{7(n+1)} .
$$

Our choice of $\nu$ comes from Proposition 6.2 Our choice of $\nu^{\prime}$ comes from the calculations of Section 7 and Section 9 (see also the footnotes in the paper). We will smooth off the indicator functions in (14) by a factor of $\varepsilon^{\nu}$, and we will in a way smooth off $\left.f\right|_{\mathfrak{z}}$ and $f_{D}$ by a factor of $\varepsilon^{\nu^{\prime}}$.

We begin our approximation procedure by first fixing a cutoff function to replace the indicator functions.

Definition 4.3 (Cutoff function). Fix $\zeta \in C^{\infty}(\mathbb{R} ;[0,1])$ such that $\zeta(x)=1$ if $x \geq$ 2 and $\zeta(x)=0$ if $x \leq 1$.

See Figure 3. We will replace the indicator $\chi_{\mathfrak{z}}$ in (14) with $\zeta\left(\mathfrak{D} / \varepsilon^{\nu}\right)$. Next, we will approximate $\left.f\right|_{3}$ and $f_{D}$. As a preliminary, we should better understand the regularity of $f$ itself, particularly near $\star$. Although Definition 2.10]ensures that $f$ is $C^{2}$ on both $\mathfrak{z}^{\circ}$ and $\Gamma$, this does not immediately enforce any regularity near $\star$. Our salvation is that we also know that $\mathscr{L} f$ is continuous, so we can use PDE theory to study the regularity of $f$ near $\star$. This is fairly easy to do on the one-dimensional manifold $\Gamma$, but it requires some more advanced PDE machinery, which we shall extract from [6], on $\mathfrak{z}$. 
Lemma 4.4. We have that $\left.f\right|_{\mathfrak{z}^{\circ}} \in H^{2}\left(\mathfrak{z}^{\circ}\right)$ and $f_{D} \in C^{2}([0, \bar{D}])$.

Next, let's extend $\left.f\right|_{\mathfrak{z}^{\circ}}$ in a PDE-based way.

Lemma 4.5. Fix an open set $Z_{1} \subset \mathbb{R}^{2}$ which contains $\mathfrak{z}$. There is a sequence $\left(\tilde{f}_{\mathfrak{z}}^{\varepsilon} ; \varepsilon>0\right)$ of elements of $C_{c}^{7}\left(Z_{1}\right)$ such that:

- $\tilde{f}_{\mathfrak{z}}^{\varepsilon}(x)=f(\star)$ for all $x \in \partial \mathfrak{z}$ and $\varepsilon>0$.

- $\lim _{\varepsilon \rightarrow 0}\left\|\tilde{f}_{\mathfrak{z}}^{\varepsilon}-f\right\|_{H^{2}\left(\mathfrak{z}^{\circ}\right)}=0$.

- $\lim _{\varepsilon \rightarrow 0}\left\|\tilde{f}_{\mathfrak{z}}^{\varepsilon}-f\right\|_{C(\mathfrak{z})}=0$.

- $\lim _{\varepsilon \rightarrow 0}\left\|\mathscr{L} \tilde{f}_{\mathfrak{z}}^{\varepsilon}-\mathscr{L}^{\dagger} f\right\|_{C(\mathfrak{z})}=0$.

- $\overline{\lim }_{\varepsilon \rightarrow 0} \varepsilon^{k \nu^{\prime}}\left\|\tilde{f}_{\mathfrak{z}}^{\varepsilon}\right\|_{C^{k}\left(Z_{1}\right)}<\infty$ for all $k \leq 7$.

Proof. See Section 9 .

We next carry out the analogous extension of $\left.f\right|_{\Gamma}$ (using the additional structure that $f \circ \pi=f_{D} \circ \mathfrak{D}$ on $\mathbf{S} \backslash \mathfrak{z}$ ). This will be done in a way parallel to that of Lemma 4.5 (even though a slightly stronger result is actually true).

Lemma 4.6. Fix an open interval $Z_{2} \subset \mathbb{R}$ which contains $[0, \bar{D}]$. There is a sequence $\left(f_{\Gamma}^{\varepsilon} ; \varepsilon>0\right)$ of elements of $C_{c}^{7}\left(Z_{2}\right)$ such that:

- $f_{\Gamma}^{\varepsilon}(0)=f(\star)$ for all $\varepsilon>0$.

- $\lim _{\varepsilon \rightarrow 0}\left\|f_{\Gamma}^{\varepsilon}-f_{D}\right\|_{C^{1}([0, \bar{D}])}=0$.

- $\lim _{\varepsilon \rightarrow 0}\left\|\mathscr{L}_{D} f_{\Gamma}^{\varepsilon}-\left(\mathscr{L}^{\dagger} f\right)_{D}\right\|_{C((0, \bar{D}))}=0$.

- $\varlimsup_{\varepsilon \rightarrow 0} \varepsilon^{k \nu^{\prime}}\left\|f_{\Gamma}^{\varepsilon}\right\|_{C^{k}\left(Z_{2}\right)}<\infty$ for all $k \leq 7$.

Proof. See Section 9 .

We can now define our approximate test function.

Definition 4.7 (Approximate test function). For $\varepsilon>0$, define

$$
\tilde{f}_{\Gamma}^{\varepsilon}(x) \stackrel{\text { def }}{=} f_{\Gamma}^{\varepsilon}(\mathfrak{D}(x))
$$

for all $x \in \mathbb{R}^{2}$ and define

$$
\tilde{f}^{\varepsilon}(x) \stackrel{\text { def }}{=} \tilde{f}_{\mathfrak{z}}^{\varepsilon}(x)\left\{1-\zeta\left(\mathfrak{D}(x) / \varepsilon^{\nu}\right)\right\}+\tilde{f}_{\Gamma}^{\varepsilon}(x) \zeta\left(\mathfrak{D}(x) / \varepsilon^{\nu}\right)
$$

for all $x \in \mathbb{R}^{2}$.

Of course one of the things which is important to us is

Lemma 4.8. We have that

$$
\lim _{\varepsilon \rightarrow 0} \sup _{x \in \overline{\mathbf{S}}}\left|\tilde{f}^{\varepsilon}(x)-f(\pi(x))\right|=0 .
$$

Proof. Fairly obvious.

The existence of the appropriate derivatives of $\tilde{f}_{\mathfrak{z}}^{\varepsilon}$ and $f_{\Gamma}^{\varepsilon}$ ensure that $\tilde{f}^{\varepsilon} \in C^{2}\left(\mathbb{R}^{2}\right)$ for each $\varepsilon>0$.

Proposition 4.9. For each $\varepsilon>0$, we have that

$$
\mathscr{L}^{\varepsilon} \tilde{f}^{\varepsilon}(x)=L^{\varepsilon}(x)+\sum_{i=1}^{3} G_{i}^{\varepsilon}(x)+K^{\varepsilon}(x), \quad x \in \mathbb{R}^{2},
$$


where

$$
\begin{aligned}
& L^{\varepsilon}(x) \stackrel{\text { def }}{=}\left\{1-\zeta\left(\mathfrak{D}(x) / \varepsilon^{\nu}\right)\right\}\left(\mathscr{L} \tilde{f}_{\mathfrak{z}}^{\varepsilon}\right)(x)+\zeta\left(\mathfrak{D}(x) / \varepsilon^{\nu}\right)\left(\mathscr{L} \tilde{f}_{\Gamma}^{\varepsilon}\right)(x), \\
& G_{1}^{\varepsilon}(x) \stackrel{\text { def }}{=}-\frac{1}{\varepsilon^{\nu}}\left\{\left\langle d \tilde{f}_{\mathfrak{z}}^{\varepsilon}, d \mathfrak{D}\right\rangle(x)-\left\langle d \tilde{f}_{\Gamma}^{\varepsilon}, d \mathfrak{D}\right\rangle(x)\right\} \dot{\zeta}\left(\mathfrak{D}(x) / \varepsilon^{\nu}\right), \\
& G_{2}^{\varepsilon}(x) \stackrel{\text { def }}{=}-\frac{1}{2 \varepsilon^{2 \nu}}\left\{\tilde{f}_{\mathfrak{z}}^{\varepsilon}(x)-\tilde{f}_{\Gamma}^{\varepsilon}(x)\right\}\langle d \mathfrak{D}, d \mathfrak{D}\rangle(x) \ddot{\zeta}\left(\mathfrak{D}(x) / \varepsilon^{\nu}\right), \\
& G_{3}^{\varepsilon}(x) \stackrel{\text { def }}{=} \varepsilon^{-2}\left(\bar{\nabla} H, \nabla \tilde{f}_{\mathfrak{z}}^{\varepsilon}\right)_{T \mathbb{R}^{2}}(x)\left\{1-\zeta\left(\mathfrak{D}(x) / \varepsilon^{\nu}\right)\right\}, \\
& K^{\varepsilon}(x) \stackrel{\text { def }}{=}-\frac{1}{\varepsilon^{\nu}}\left\{\tilde{f}_{\mathfrak{z}}^{\varepsilon}(x)-\tilde{f}_{\Gamma}^{\varepsilon}(x)\right\} \dot{\zeta}\left(\mathfrak{D}(x) / \varepsilon^{\nu}\right)(\mathscr{L} \mathfrak{D})(x)
\end{aligned}
$$

for all $x \in \overline{\mathbf{S}}$.

Proof. A straightforward calculation.

Recall that our goal is to show that $\mathbb{P}^{\dagger}$ satisfies (5). Let's indicate how we get there in a number of steps. The hard work will be done in the next several sections. First, we claim that the limit of the $L^{\varepsilon}$ term is the desired generator.

Proposition 4.10. Fix $t>0$. Then

$$
\lim _{\varepsilon \rightarrow 0} \mathbb{E}^{\varepsilon}\left[\left|\int_{0}^{t \wedge \mathfrak{e}}\left\{L^{\varepsilon}\left(X_{s}\right)-\left(\mathscr{L}^{\dagger} f\right)\left(Y_{s}\right)\right\} d s\right|\right]=0 .
$$

Proof. Given in Section 7.

We next claim that the effect of the $G_{i}^{\varepsilon}$ 's is negligible; the proof shows that this is true for $i \in\{1,2\}$ exactly because of the glueing conditions.

Proposition 4.11. Fix times $0 \leq r_{1}<r_{2}<\cdots<r_{n} \leq s<t$ and test functions $\left\{\varphi_{j} ; j=1,2 \ldots n\right\} \subset C_{b}\left(\mathbb{R}^{2}\right)$. Then

$$
\lim _{\varepsilon \rightarrow 0} \mathbb{E}^{\varepsilon}\left[\int_{s \wedge \mathfrak{e}}^{t \wedge \mathfrak{e}} G_{i}^{\varepsilon}\left(X_{u}\right) d u \prod_{j=1}^{n} \varphi_{j}\left(X_{r_{j}}\right)\right]=0
$$

for $i \in\{1,2,3\}$.

Proof. Lemmas 7.1 and 7.2

We thirdly claim that $K^{\varepsilon}$ is negligible; the main idea is that $X$ does not spend too much time near $\partial \mathfrak{z}$.

Proposition 4.12. Fix $t>0$. Then

$$
\lim _{\varepsilon \rightarrow 0} \mathbb{E}^{\varepsilon}\left[\left|\int_{0}^{t \wedge \mathfrak{e}} K^{\varepsilon}\left(X_{s}\right) d s\right|\right]=0 .
$$

Proof. Given in Section 7 .

The combination of Propositions 4.104 .12 is

Proposition 4.13. Fix $f \in \mathscr{D}^{\dagger}$ and $t>0$. Then

$$
\lim _{\varepsilon \rightarrow 0} \mathbb{E}^{\varepsilon}\left[\int_{0}^{t \wedge \mathfrak{e}}\left\{\left(\mathscr{L}^{\varepsilon} \tilde{f}^{\varepsilon}\right)\left(X_{s}\right)-\left(\mathscr{L}^{\dagger} f\right)\left(Y_{s}\right)\right\} d s\right]=0 .
$$

Proof. Obvious.

We also can give the 
Proof of Proposition 4.1. Combine Lemma 4.8 and Proposition 4.13 with the stopped martingale problem of Definition 2.3. We note that by the definition of $\mathscr{D}^{\dagger}$ (viz., that $\left.\left(\mathscr{L}^{\dagger} f\right)(\circledast)=0\right)$,

$$
\int_{0}^{t}\left(\mathscr{L}^{\dagger} f\right)\left(Y_{s}\right) d s=\int_{0}^{t \wedge \mathfrak{e}}\left(\mathscr{L}^{\dagger} f\right)\left(Y_{s}\right) d s
$$

We also note that $\mathbb{P}^{\dagger}\left\{X_{0}^{\dagger}=\left[x_{\circ}\right]\right\} \geq \varlimsup_{\varepsilon \rightarrow 0} \mathbb{P}^{\varepsilon}\left\{Y_{0}=\left[x_{\circ}\right]\right\}=1$.

\section{Residence Time}

Here we show that $X$ does not spend too much time (under $\mathbb{P}^{\varepsilon}$ ) near $\partial \mathfrak{z}$. We will directly use this to prove Proposition 4.12. It will also help us prove Propositions 4.10 and 4.11

Proposition 5.1. Fix $t>0$. Then

$$
\varlimsup_{\varepsilon, \delta \rightarrow 0} \mathbb{E}^{\varepsilon}\left[\int_{0}^{t \wedge \mathfrak{e}} \frac{1}{\delta} \chi_{[-\delta, \delta]}\left(\mathfrak{D}\left(X_{s}\right)\right) d s\right]<\infty .
$$

Proof. Define

$$
u_{\delta}(d) \stackrel{\text { def }}{=} \int_{s=0}^{d}(d-s) \frac{1}{\delta} \chi_{[-\delta, \delta]}(s) d s, \quad d \in \mathbb{R}
$$

then

$$
\ddot{u}_{\delta}(d)=\frac{1}{\delta} \chi_{[-\delta, \delta]}(d)
$$

for all $d \in \mathbb{R}$ and there is a $C_{1}>0$ such that

$$
\left|u_{\delta}(d)\right| \leq C_{1} \quad \text { and } \quad\left|\dot{u}_{\delta}(d)\right| \leq C_{1}
$$

for all $d \in[-\bar{D}, \bar{D}]$ and all $0<\delta<1$. The martingale problem tells us that for $0 \leq t \leq \mathfrak{e}$,

$$
\begin{aligned}
u_{\delta}\left(\mathfrak{D}\left(X_{t}\right)\right)=u_{\delta}\left(\mathfrak{D}\left(X_{0}\right)\right)+ & \int_{0}^{t} \dot{u}_{\delta}\left(\mathfrak{D}\left(X_{s}\right)\right) \mathscr{L} \mathfrak{D}\left(X_{s}\right) d s \\
& +\frac{1}{2} \int_{0}^{t} \frac{1}{\delta} \chi_{[-\delta, \delta]}\left(\mathfrak{D}\left(X_{s}\right)\right)\left\langle d \mathfrak{D}\left(X_{s}\right), d \mathfrak{D}\left(X_{s}\right)\right\rangle d s+N_{t}
\end{aligned}
$$

where $N$ is a $\mathbb{P}^{\varepsilon}$-martingale. Recalling now the neighborhood $\mathcal{N}$ defined in (6), we set

$$
C_{2} \stackrel{\text { def }}{=} \frac{1}{2} \inf _{x \in \mathcal{N}}\langle d \mathfrak{D}(x), d \mathfrak{D}(x)\rangle>0 \quad \text { and } \quad C_{3} \stackrel{\text { def }}{=} 2 \sup _{x \in \mathcal{N}}|\mathscr{L} \mathfrak{D}(x)|<\infty .
$$

We thus have that for $0 \leq t \leq \mathfrak{e}$ and $\delta<\min \{\varkappa, 1\}$,

$$
2 C_{1} \geq u_{\delta}\left(\mathfrak{D}\left(X_{t}\right)\right)-u_{\delta}\left(\mathfrak{D}\left(X_{0}\right)\right) \geq-C_{1} C_{3} t+C_{2} \int_{0}^{t} \frac{1}{\delta} \chi_{[-\delta, \delta]}\left(\mathfrak{D}\left(X_{s}\right)\right) d s+N_{t}
$$

and this easily implies the result.

\section{Stochastic Averaging}

Our goal here is to prove the basic results of stochastic averaging. First, we will average away from $\partial \mathfrak{z}$, and then we will average in a boundary layer near $\partial \mathfrak{z}$. 
6.1. Averaging Slightly Away from the Boundary. Here we will prove an averaging result for functions whose support is slightly away from $\mathfrak{z}$. In order to do so, we use the specific structure of $H$ (given in Assumption 2.4) to ensure that the averaging calculations, when written in terms of $\mathfrak{D}$, are regular near $\partial \mathfrak{z}$. This will also play a role in our later considerations of averaging at $\partial \mathfrak{z}$.

To begin, define the flow

$$
\begin{aligned}
& \dot{\mathfrak{g}}_{t}^{D}(x)=\bar{\nabla} D\left(\mathfrak{g}_{t}^{D}(x)\right), \\
& \mathfrak{g}_{0}^{D}(x)=x,
\end{aligned}
$$

then by (2), $\mathfrak{g}_{t}(x)=\mathfrak{g}_{n \mathfrak{D}^{n-1}(x) t}^{D}(x)$ for all $x \in \overline{\mathbf{S}} \backslash \mathfrak{z}$ and all $t \in \mathbb{R}$, where $\mathfrak{g}$ is as in $(\underline{3})$. Define also $\mathfrak{T}^{D}:(-\varkappa, \bar{D}] \rightarrow \mathbb{R}$ by

$$
\begin{aligned}
\mathfrak{T}^{D}(\mathfrak{D}(x)) & =\inf \left\{t>0: \mathfrak{g}_{t}^{D}(x)=x\right\} \\
& =\int_{y \in \mathfrak{D}^{-1}(x)}\|\bar{\nabla} \mathfrak{D}(y)\|_{T \mathbb{R}^{2}}^{-1} \mathscr{H}^{1}(d y)
\end{aligned}
$$

for each $x \in \overline{\mathbf{S}}$ such that $\mathfrak{D}(x)>-\varkappa$; it is easy to see that $\mathfrak{T}^{D}$ is indeed well-defined and finite on $(-\varkappa, \bar{D}]$. For any $\phi \in B(\overline{\mathbf{S}})$, define $\mathbf{A}^{D} \phi \in B((-\varkappa, \bar{D}])$ by

$$
\left(\mathbf{A}^{D} \phi\right)(\mathfrak{D}(x))=\frac{1}{\mathfrak{T}^{D}(\mathfrak{D}(x))} \int_{0}^{\mathfrak{T}^{D}(\mathfrak{D}(x))} \phi\left(\mathfrak{g}_{s}^{D}(x)\right) d s
$$

for all $x \in \overline{\mathbf{S}}$ such that $\mathfrak{D}(x)>-\varkappa$ (again, it is easy to see that $\mathbf{A}^{D} \phi$ is well-defined). We note that if $\phi \in B(\overline{\mathbf{S}})$, then $\phi$ is in the domain of $\mathbf{A}^{\circ}$ and

$$
\left(\mathbf{A}^{D} \phi\right)(\mathfrak{D}(x))=\left(\mathbf{A}^{\circ} \phi\right)([x])
$$

for $x \in \mathbf{S} \backslash \mathfrak{z}$. Finally, define

$$
b_{\eta}(\vartheta) \stackrel{\text { def }}{=} \max \{0, \vartheta\}^{\eta}, \quad \vartheta \in \mathbb{R},
$$

then $H=b_{n}(\mathfrak{D})$ and we can rewrite (2) as $\bar{\nabla} H=n b_{n-1}(\mathfrak{D}) \bar{\nabla} \mathfrak{D}$.

Our first averaging result is

Lemma 6.1. Let $\varpi \in C_{c}^{\infty}(\mathbb{R})$ be such that $\operatorname{supp} \varpi \subset(0, \infty)$. Fix $p>0$. Then there is a constant $C>0$ such that for any $\phi \in C^{2}(\overline{\mathbf{S}})$,

$$
\mathbb{E}^{\varepsilon}\left[\left|\int_{0}^{t \wedge \mathfrak{e}}\left\{\phi\left(X_{s}\right)-\left(\mathbf{A}^{\circ} \phi\right)\left(\left[X_{s}\right]\right)\right\} \varpi\left(\mathfrak{D}\left(X_{s}\right) / \varepsilon^{p}\right) d s\right|\right] \leq \varepsilon^{2-p(n+1)} C(1+t)\|\phi\|_{C^{2}(\overline{\mathbf{S}})}
$$

for all $\varepsilon>0$.

Proof. Define

$$
\Phi(x) \stackrel{\text { def }}{=} \frac{1}{\mathfrak{T}^{D}(\mathfrak{D}(x))} \int_{0}^{\mathfrak{T}^{D}(\mathfrak{D}(x))} s \phi\left(\mathfrak{g}_{s}^{D}(x)\right) d s
$$

for all $x \in \overline{\mathbf{S}}$ such that $\mathfrak{D}(x)>-\varkappa$. Then

$$
\Phi\left(\mathfrak{g}_{t}^{D}(x)\right) \stackrel{\text { def }}{=} \frac{1}{\mathfrak{T}^{D}(\mathfrak{D}(x))} \int_{t}^{\mathfrak{T}^{D}(\mathfrak{D}(x))+t}(s-t) \phi\left(\mathfrak{g}_{s}^{D}(x)\right) d s
$$

for all such $x$. Differentiating this with respect to $t$, we see that

$$
(\bar{\nabla} D \Phi)(x)=\phi(x)-\left(\mathbf{A}^{D} \phi\right)(\mathfrak{D}(x))
$$


for all $x \in \overline{\mathbf{S}}$ such that $\mathfrak{D}(x)>-\varkappa$. Set next

$$
\tilde{\Phi}^{\varepsilon}(x) \stackrel{\text { def }}{=} \frac{1}{n b_{n-1}(\mathfrak{D}(x))} \Phi(x) \varpi\left(\mathfrak{D}(x) / \varepsilon^{p}\right)
$$

for all $x \in \overline{\mathbf{S}}$; it is easy to see that this is well-defined on all of $\mathbb{R}^{2}$ (since supp $\varpi \subset$ $(0, \infty))$. Then (using (2) and (17))

$$
\left(\bar{\nabla} H \tilde{\Phi}^{\varepsilon}\right)(x)=\left\{\phi(x)-\left(\mathbf{A}^{\circ} \phi\right)([x])\right\} \varpi\left(\mathfrak{D}(x) / \varepsilon^{p}\right), \quad x \in \overline{\mathbf{S}}
$$

Some simple calculations (which use the fact that the support of $\varpi$ is strictly contained in $(0, \infty))$ ensure that there is a constant $C>0$ such that

$$
\left\|\tilde{\Phi}^{\varepsilon}\right\|_{C^{2}(\overline{\mathbf{S}})} \leq C \varepsilon^{-p(n+1)}\|\phi\|_{C^{2}(\overline{\mathbf{S}})}
$$

The martingale problem thus tells us that

$$
\begin{aligned}
\int_{0}^{t \wedge \mathfrak{e}}\left\{\phi\left(X_{s}\right)-\left(\mathbf{A}^{\circ} \phi\right)\left(\left[X_{s}\right]\right)\right\} \varpi\left(\mathfrak{D}\left(X_{s}\right) / \varepsilon^{p}\right) d s & =\varepsilon^{2}\left\{\tilde{\Phi}^{\varepsilon}\left(X_{t \wedge \mathfrak{e}}\right)-\tilde{\Phi}^{\varepsilon}\left(X_{0}\right)\right\} \\
& -\varepsilon^{2} \int_{0}^{t \wedge \mathfrak{e}}\left(\mathscr{L} \tilde{\Phi}^{\varepsilon}\right)\left(X_{s}\right) d s-\varepsilon^{2} M_{t \wedge \mathfrak{e}}
\end{aligned}
$$

where $M$ is a $\mathbb{P}^{\varepsilon}$-martingale with quadratic variation

$$
\langle M\rangle_{t}=\int_{0}^{t}\left\langle d \tilde{\Phi}^{\varepsilon}, d \tilde{\Phi}^{\varepsilon}\right\rangle\left(X_{s}\right) d s, \quad 0 \leq t \leq \mathfrak{e} .
$$

Using (21), we get the stated result.

6.2. Stochastic Averaging Near the Boundary. We next need to carry the idea of stochastic averaging in to $\partial \mathfrak{z}$.

Proposition 6.2. Fix $\varpi \in C_{c}^{\infty}(\mathbb{R})$. Fix times $0 \leq r_{1}<r_{2}<\cdots<r_{n} \leq s<t$ and test functions $\left\{\varphi_{j} ; j=1,2 \ldots n\right\} \subset C_{b}\left(\mathbb{R}^{2}\right)$. Then there is a $C>0$ such that for any $\phi \in C^{4}\left(\mathbb{R}^{2}\right)$,

$$
\begin{aligned}
& \varepsilon^{-2}\left|\mathbb{E}^{\varepsilon}\left[\int_{s \wedge \mathfrak{e}}^{t \wedge \mathfrak{e}} b_{n}\left(\mathfrak{D}\left(X_{u}\right)\right) \varpi\left(\mathfrak{D}\left(X_{u}\right) / \varepsilon^{\nu}\right)\left\{\phi\left(X_{u}\right)-\left(\mathbf{A}^{D} \phi\right)\left(X_{u}\right)\right\} d u \prod_{j=1}^{n} \varphi_{j}\left(X_{r_{j}}\right)\right]\right| \\
& \leq C(1+t) \varepsilon^{\nu}\|\phi\|_{C^{4}\left(\mathbb{R}^{2}\right)}
\end{aligned}
$$

for all $\varepsilon>0$, where $b_{n}$ is as in (18).

Remark 6.3. Note that if $\mathfrak{D}(x)$ is on order $\varepsilon^{\nu}$, then $\varepsilon^{-2} b_{n}(\mathfrak{D}(x))$ is only of order $\varepsilon^{-2+\nu n}$. The residence-time result of Proposition 5.1 thus would only give us that for some $C>1$

$$
\begin{array}{r}
\varepsilon^{-2}\left|\mathbb{E}^{\varepsilon}\left[\int_{s \wedge \mathfrak{e}}^{t \wedge \mathfrak{e}} b_{n}\left(\mathfrak{D}\left(X_{u}\right)\right) \varpi\left(\mathfrak{D}\left(X_{u}\right) / \varepsilon^{\nu}\right)\left\{\phi\left(X_{u}\right)-\left(\mathbf{A}^{D} \phi\right)\left(X_{u}\right)\right\} d u \prod_{j=1}^{n} \varphi_{j}\left(X_{r_{j}}\right)\right]\right| \\
\leq C\|\phi\|_{C(\overline{\mathbf{S}})} \varepsilon^{-2+\nu n} \mathbb{E}^{\varepsilon}\left[\int_{s \wedge \mathfrak{e}}^{t \wedge \mathfrak{e}}\left|\varpi\left(\mathfrak{D}\left(X_{u}\right) / \varepsilon^{\nu}\right)\right| d u\right] \\
\leq C^{2} \varepsilon^{-2+\nu n+\nu}=C^{2} \varepsilon^{\nu(n+1)-2}=C^{2}
\end{array}
$$


(remember from Definition 4.2 that $\nu=2 /(n+1)$ ). The point of Proposition 6.2 is that averaging gives us just a bit more, namely an extra power of $\varepsilon^{\nu}$.

Remark 6.4 (PDE for Glueing Condition). We note that the absolute value signs in (22) are outside the expectation (whereas in Lemma 6.1 they were inside). This reflects the fact that this result is essentially a martingale result. To see this, define

$$
\bar{G}^{\varepsilon}(x) \stackrel{\text { def }}{=} \varepsilon^{-2} b_{n}(\mathfrak{D}(x)) \varpi\left(\mathfrak{D}(x) / \varepsilon^{\nu}\right)\left\{\phi(x)-\left(\mathbf{A}^{D} \phi\right)(x)\right\}, \quad x \in \overline{\mathbf{S}} .
$$

Suppose that for each $\varepsilon>0$, we can find $\Psi^{\varepsilon} \in C^{2}\left(\mathbb{R}^{2}\right)$ such that

$$
\mathscr{L}^{\varepsilon} \Psi^{\varepsilon} \approx \bar{G}^{\varepsilon}
$$

on $\overline{\mathbf{S}}$. Suppose furthermore that

$$
\lim _{\varepsilon \rightarrow 0} \varepsilon^{-\nu}\left\|\Psi^{\varepsilon}\right\|_{C(\overline{\mathbf{S}})}<\infty .
$$

Then the martingale problem tells us that

$$
\begin{aligned}
\int_{s \wedge \mathfrak{e}}^{t \wedge \mathfrak{e}} \bar{G}^{\varepsilon}\left(X_{u}\right) d u \approx & \int_{s \wedge \mathfrak{e}}^{t \wedge \mathfrak{e}}\left(\mathscr{L}^{\varepsilon} \Psi^{\varepsilon}\right)\left(X_{u}\right) d u \\
& =\left\{\Psi^{\varepsilon}\left(X_{t \wedge \mathfrak{e}}\right)-\Psi^{\varepsilon}\left(X_{s \wedge \mathfrak{e}}\right)\right\}+M_{t \wedge \mathfrak{e}}-M_{s \wedge \mathfrak{e}} \approx M_{t \wedge \mathfrak{e}}-M_{s \wedge \mathfrak{e}}
\end{aligned}
$$

where $M$ is a $\mathbb{P}^{\varepsilon}$-martingale. By optional sampling, we then have that

$$
\mathbb{E}^{\varepsilon}\left[\left(M_{t \wedge \mathfrak{e}}-M_{s \wedge \mathfrak{e}}\right) \mid \mathscr{F}_{s}\right]=0,
$$

which would then finish the proof. Our honest technical goal is consequently to approximately solve the PDE (24) with sufficient accuracy. We note that the operator $\varepsilon^{2} \mathscr{L}^{\varepsilon}$ has an order-one drift and a small diffusive term. Thus, we have a singular perturbation problem.

Central to our arguments will be some Khasminskii coordinates. First, let $\mathcal{C}$ be a curve in $\mathcal{N}$ which crosses all of the $\mathfrak{D}^{-1}(d)$ 's in $\mathcal{N}$ transversally. To be specific about this, fix $x^{*} \in \partial \mathfrak{z}$, and let

$$
\gamma(t) \stackrel{\text { def }}{=} \underline{\phi}_{t}\left(x^{*}\right)
$$

for all $t \in(-\varkappa, \varkappa)$, where $\left\{\underline{\phi}_{t} ; t \in \mathbb{R}\right\}$ satisfies (46) (thus $\mathfrak{D}(\gamma(t))=-t$ for all $t \in(-\varkappa, \varkappa))$. Set $\mathcal{C} \stackrel{\text { def }}{=} \gamma(-\varkappa, \varkappa)$; then

$$
\mathcal{N}=\left\{\mathfrak{g}_{t}^{D}(x): x \in \mathcal{C}, 0 \leq t<\mathfrak{T}^{D}(\mathfrak{D}(x))\right\} .
$$

For each $x \in \mathcal{C}$, define

$$
\Theta\left(\mathfrak{g}_{t}^{D}(x)\right)=\int_{0}^{t}\langle d \mathfrak{D}, d \mathfrak{D}\rangle\left(\mathfrak{g}_{s}^{D}(x)\right) d s
$$

for all $0 \leq t<\mathfrak{T}^{D}(\mathfrak{D}(x))$. For each $d \in(-\varkappa, \varkappa)$, define

$$
\begin{aligned}
\alpha(d) & \stackrel{\text { def }}{=} \sup \{\Theta(x): \mathfrak{D}(x)=d\} \\
& =\int_{x \in \mathfrak{D}^{-1}(d)} \frac{\langle d \mathfrak{D}, d \mathfrak{D}\rangle(x)}{\|\bar{\nabla} \mathfrak{D}(x)\|_{T \mathbb{R}^{2}}} \mathscr{H}^{1}(d x) .
\end{aligned}
$$

It is clear from the second representation that $\alpha$ is $C^{\infty}$ on $(-\varkappa, \varkappa)$ and that $\inf _{-\varkappa<d<\varkappa} \alpha(d)>0$. Then the map $x \mapsto(\Theta(x), \mathfrak{D}(x))$ is a homeomorphism from $\mathcal{N}$ to $\left\{(\theta, d) \in \mathbb{R}^{2}:-\varkappa<d<\varkappa, 0 \leq \theta<\alpha(d)\right\}$. It is also clear that

$$
(\bar{\nabla} D, \nabla \Theta)_{T \mathbb{R}^{2}}(x)=\langle d \mathfrak{D}, d \mathfrak{D}\rangle(x)
$$


for all $x \in \mathcal{N} \backslash \mathcal{C}$. This will be crucial; informally, it means that equal increments of $\Theta$ correspond to equal amounts of diffusion across $\partial \mathfrak{z}$.

Let's now get back to the issues raised by Remark [6.4. Instead of deductively identifying how to find the $\Psi^{\varepsilon}$ described there, we will develop the answer. The reasoning leading to the answer may be easily identified by reading our arguments backwards.

First, define $\Phi$ as in (19). Let's convert $\Phi$ to Khasminskii coordinates. There is a $\tilde{\Phi} \in C^{4}(\mathbb{R} \times \mathbb{R})$ such that

$$
\begin{aligned}
\Phi(x) & =\tilde{\Phi}(\Theta(x), \mathfrak{D}(x)), \quad x \in \mathcal{N}, \\
\tilde{\Phi}(\theta+\alpha(d), d) & =\tilde{\Phi}(\theta, d), \quad \theta \in \mathbb{R},|d|<\varkappa .
\end{aligned}
$$

The combination of (20) and (25) imply that

$$
\phi(x)-\left(\mathbf{A}^{D} \phi\right)(x)=(\bar{\nabla} \mathfrak{D} \Phi)(x)=\frac{\partial \tilde{\Phi}}{\partial \theta}(\Theta(x), \mathfrak{D}(x))\langle d \mathfrak{D}, d \mathfrak{D}\rangle(x)
$$

for all $x \in \mathcal{N} \backslash \mathcal{C}$ and thus that

$$
\begin{aligned}
\bar{G}^{\varepsilon}(x) & =\varepsilon^{-2} b_{n}(\mathfrak{D}(x)) \frac{\partial \tilde{\Phi}}{\partial \theta}(\Theta(x), \mathfrak{D}(x)) \varpi\left(\frac{\mathfrak{D}(x)}{\varepsilon^{\nu}}\right)\langle d \mathfrak{D}, d \mathfrak{D}\rangle(x) \\
& =\varepsilon^{-2+\nu} b_{n-1}(\mathfrak{D}(x)) \frac{\partial \tilde{\Phi}}{\partial \theta}(\Theta(x), \mathfrak{D}(x)) \tilde{\varpi}\left(\frac{b_{1 / \nu}(\mathfrak{D}(x))}{\varepsilon}\right)\langle d \mathfrak{D}, d \mathfrak{D}\rangle(x)
\end{aligned}
$$

for $x \in \mathcal{N} \backslash \mathcal{C}$, where

$$
\tilde{\varpi}(d) \stackrel{\text { def }}{=} \varpi\left(d^{\nu}\right) d^{\nu}
$$

for all $d \in \mathbb{R}$. We write $b_{n}(d)$ as $b_{n-1}(d) d$ in deference to the fact that $\bar{\nabla} H=$ $n b_{n-1} \bar{\nabla} \mathfrak{D}$. We prove the following result in Section 10 .

Proposition 6.5. There is a $\tilde{\Psi} \in C^{2}\left(\mathbb{R} \times \mathbb{R}_{+}\right)$such that

$$
\begin{gathered}
\tilde{\Psi}(\theta+\alpha(0), d)=\tilde{\Psi}(\theta, d), \\
n \frac{\partial \tilde{\Psi}}{\partial \theta}(\theta, d)+\frac{(n+1)^{2}}{8} \frac{\partial^{2} \tilde{\Psi}}{\partial d^{2}}(\theta, d)+\frac{n^{2}-1}{8 d} \frac{\partial \tilde{\Psi}}{\partial d}(\theta, d)=\frac{\partial \tilde{\Phi}}{\partial \theta}(\theta, 0) \tilde{\varpi}(d)
\end{gathered}
$$

for all $(\theta, d) \in \mathbb{R} \times(0, \infty)$ and

$$
\frac{\partial \tilde{\Psi}}{\partial d}(\theta, 0)=0
$$

for all $\theta \in \mathbb{R}$ and such that for some $C>0$ (not depending on $\tilde{\Phi}$ )

$$
\left|D^{\alpha} \tilde{\Psi}(\theta, d)\right| \leq C\|\tilde{\Phi}\|_{C^{4}(\mathbb{R} \times\{0\})} e^{-d / C}
$$

for all $(\theta, d) \in \mathbb{R} \times(0, \infty)$ and for any multi-index $\alpha$ of degree 2 or less,

Proof. Given in Section 10,

Note the $d$ in the denominator of the third term on the left-hand side of the PDE for $\tilde{\Psi}$. Note also that by (19) and (26) there is a constant $C>0$ which does not depend on $\phi$ such that

$$
\|\tilde{\Phi}\|_{C^{4}(\mathbb{R} \times\{0\})} \leq C\|\phi\|_{C^{4}(\mathcal{N})} .
$$


Define

$$
\Theta^{\circ}(x) \stackrel{\text { def }}{=} \frac{\alpha(0)}{\alpha(\mathfrak{D}(x))} \Theta(x)
$$

for all $x \in \mathcal{N}$. We have

Lemma 6.6. Let $\tilde{\Psi}$ be as in Proposition 6.5 and define

$$
\Psi^{\varepsilon}(x) \stackrel{\text { def }}{=} \varepsilon^{\nu} \tilde{\Psi}\left(\Theta^{\circ}(x), \frac{b_{1 / \nu}(\mathfrak{D}(x))}{\varepsilon}\right), \quad|\mathfrak{D}(x)|<\varkappa,
$$

for all $x \in \mathcal{N}$. Then for each $\varepsilon>0$,

i) $\Psi^{\varepsilon} \in C^{1}(\mathcal{N})$,

ii) $\Psi^{\varepsilon}$ is $C^{2}$ on $\mathcal{N} \backslash\left(\partial_{\mathfrak{z}} \cup \mathcal{C}\right)$,

iii) $\mathscr{L}^{\varepsilon} \Psi^{\varepsilon}(x) \stackrel{\text { def }}{=} \lim _{y \in \mathcal{N} \backslash(\partial \mathfrak{z} \cup \mathcal{C})} \mathscr{L}^{\varepsilon} \Psi^{\varepsilon}(y)$ exists for all $x \in \partial \mathfrak{z} \cup \mathcal{C}$.

Furthermore there is a constant $C>0$ such that

$$
\begin{aligned}
\left|\left(\mathscr{L}^{\varepsilon} \Psi^{\varepsilon}\right)(x)-\bar{G}^{\varepsilon}(x)\right| & \leq C\|\phi\|_{C^{4}(\mathcal{N})} \exp \left[-C^{-1}|\mathfrak{D}(x)|^{1 / \nu} / \varepsilon\right], \\
\sqrt{\left\langle d \Psi^{\varepsilon}, d \Psi^{\varepsilon}\right\rangle(x)} & \leq C\|\phi\|_{C^{4}(\mathcal{N})} \exp \left[-C^{-1}|\mathfrak{D}(x)|^{1 / \nu} / \varepsilon\right], \\
\left|\Psi^{\varepsilon}(x)\right| & \leq C \varepsilon^{\nu}\|\phi\|_{C^{4}(\mathcal{N})}
\end{aligned}
$$

for all $x \in \mathcal{N} \backslash(\partial \mathfrak{z} \cup \mathcal{C})$ and $\varepsilon>0$.

The definition of $\Psi^{\varepsilon}$ can more explicitly be given as

$$
\Psi^{\varepsilon}(x)= \begin{cases}\varepsilon^{\nu} \tilde{\Psi}\left(\Theta^{\circ}(x), 0\right) & \text { if }-\varkappa<\mathfrak{D}(x)<0, \\ \varepsilon^{\nu} \tilde{\Psi}\left(\Theta^{\circ}(x), \frac{\mathfrak{D}^{(n+1) / 2}(x)}{\varepsilon}\right) & \text { if } 0 \leq \mathfrak{D}(x)<\varkappa .\end{cases}
$$

Proof of Lemma 6.6. Clearly $\Psi^{\varepsilon}$ is $C^{\infty}$ on $\mathcal{N} \backslash\left(\partial_{\mathfrak{z}} \cup \mathcal{C}\right)$. In view of the continuity of $b_{1 / \nu}$ and the periodicity of $\tilde{\Psi}$, it is fairly easy to see that $\Psi^{\varepsilon}$ has a limit at each point of $\partial \mathfrak{z} \cup \mathcal{C}$, so $\Psi^{\varepsilon}$ is continuous on all of $\mathcal{N}$. We next need to show that all first-order derivatives tend to a limit at each point of $\partial \mathfrak{z} \cup \mathcal{C}$. Note that $\bar{\nabla} \mathfrak{D}(x)$ and $\nabla \mathfrak{D}(x)$ span all of $T_{x} \mathbb{R}^{2}$ for each $x \in \mathcal{N}$. From the periodicity and boundary conditions of $\tilde{\Psi}$, we also see that $\nabla \mathfrak{D} \Psi^{\varepsilon}$ and $\bar{\nabla} \mathfrak{D} \Psi^{\varepsilon}$ both have limits at each point of $\partial \mathfrak{z} \cup \mathcal{C}$; thus $\Psi^{\varepsilon} \in C^{1}(\mathcal{N})$ (note that $b_{1 / \nu}$ is differentiable since $1 / \nu-1=(n-1) / 2>0$ ). Thus claims i) and ii) are true. Next, let's compute $\mathscr{L}^{\varepsilon} \Psi^{\varepsilon}$. We see that

$$
\begin{aligned}
&\left(\mathscr{L}^{\varepsilon} \Psi^{\varepsilon}\right)(x)= n \varepsilon^{\nu-2} b_{n-1}(\mathfrak{D}(x)) \frac{\alpha(0)}{\alpha(\mathfrak{D}(x))} \frac{\partial \tilde{\Psi}}{\partial \theta}(\bar{\nabla} \mathfrak{D} \Theta)(x) \\
&+ \frac{\nu^{-2}}{2} \varepsilon^{\nu-2} b_{1 / \nu-1}^{2}(\mathfrak{D}(x)) \frac{\partial^{2} \tilde{\Psi}}{\partial d^{2}}\langle d \mathfrak{D}, d \mathfrak{D}\rangle(x) \\
&+\frac{\nu^{-1}\left(\nu^{-1}-1\right)}{2} \varepsilon^{\nu-1} b_{1 / \nu-2}(\mathfrak{D}(x)) \frac{\partial \tilde{\Psi}}{\partial d}\langle d \mathfrak{D}, d \mathfrak{D}\rangle(x)+I_{1}^{\varepsilon}(x)+I_{2}^{\varepsilon}(x) \\
&=\varepsilon^{\nu-2} b_{n-1}(\mathfrak{D}(x))\langle d \mathfrak{D}, d \mathfrak{D}\rangle(x)\left\{n \frac{\alpha(0)}{\alpha(\mathfrak{D}(x))} \frac{\partial \tilde{\Psi}}{\partial \theta}+\frac{(n+1)^{2}}{8} \frac{\partial^{2} \tilde{\Psi}}{\partial d^{2}}\right. \\
&\left.+\frac{n^{2}-1}{8}\left(\frac{b_{1 / \nu}(\mathfrak{D}(x))}{\varepsilon}\right)^{-1} \frac{\partial \tilde{\Psi}}{\partial d}\right\}+I_{1}^{\varepsilon}(x)+I_{2}^{\varepsilon}(x)
\end{aligned}
$$


for all $x \in \mathcal{N} \backslash\left(\partial_{\mathfrak{z}} \cup \mathcal{C}\right)$, where

$$
\begin{aligned}
& I_{1}^{\varepsilon}(x) \stackrel{\text { def }}{=} \nu^{-1} \varepsilon^{\nu-1} b_{1 / \nu-1}(\mathfrak{D}(x))\left\{\frac{1}{2} \frac{\partial \tilde{\Psi}}{\partial d}(\mathscr{L} \mathfrak{D})(x)+\frac{\partial^{2} \tilde{\Psi}}{\partial d \partial \theta}\left\langle d \Theta^{\circ}, d \mathfrak{D}\right\rangle(x)\right\}, \\
& I_{2}^{\varepsilon}(x) \stackrel{\text { def }}{=} \varepsilon^{\nu}\left\{\frac{\partial \tilde{\Psi}}{\partial \theta}\left(\mathscr{L} \Theta^{\circ}\right)(x)+\frac{1}{2} \frac{\partial^{2} \tilde{\Psi}}{\partial \theta^{2}}\left\langle d \Theta^{\circ}, d \Theta^{\circ}\right\rangle(x)\right\}
\end{aligned}
$$

where in both (28) and (29), $\tilde{\Psi}$ and its various derivatives are all evaluated at $\left(\Theta^{\circ}(x), b_{1 / \nu}(\mathfrak{D}(x)) / \varepsilon\right)$. Thus

$$
\left(\mathscr{L}^{\varepsilon} \Psi^{\varepsilon}\right)(x)=\bar{G}^{\varepsilon}(x)+\sum_{i=1}^{4} I_{i}^{\varepsilon}(x)
$$

for all $x \in \mathcal{N} \backslash(\partial \mathfrak{z} \cup \mathcal{C})$, where

$$
\begin{aligned}
I_{3}^{\varepsilon}(x) \stackrel{\text { def }}{=} \varepsilon^{\nu-2} b_{n-1}(\mathfrak{D}(x))\langle d \mathfrak{D}, d \mathfrak{D}\rangle(x) & n \frac{\alpha(0)-\alpha(\mathfrak{D}(x))}{\alpha(\mathfrak{D}(x))} \frac{\partial \tilde{\Psi}}{\partial \theta}\left(\Theta^{\circ}(x), \frac{b_{1 / \nu}(\mathfrak{D}(x))}{\varepsilon}\right), \\
I_{4}^{\varepsilon}(x) \stackrel{\text { def }}{=} \varepsilon^{\nu-2} b_{n-1}(\mathfrak{D}(x))\langle d \mathfrak{D}, d \mathfrak{D}\rangle(x) & \left\{\frac{\partial \tilde{\Phi}}{\partial \theta}\left(\Theta^{\circ}(x), 0\right)\right. \\
& \left.-\frac{\partial \tilde{\Phi}}{\partial \theta}(\Theta(x), \mathfrak{D}(x))\right\} \tilde{\varpi}\left(\frac{b_{1 / \nu}(\mathfrak{D}(x))}{\varepsilon}\right)
\end{aligned}
$$

for all $x \in \mathcal{N} \backslash\left(\partial_{\mathfrak{z}} \cup \mathcal{C}\right)$. From this we clearly see that $\lim _{y \in \mathcal{N} \backslash\left(\partial_{\mathfrak{z}} \cup \mathcal{C}\right)} \mathscr{L}^{\varepsilon} \Psi^{\varepsilon}(y)$ exists for all $x \in \partial \mathfrak{z} \cup \mathcal{C}$ (the fact that $\tilde{\Psi}$ is $C^{2}$ at $\mathbb{R} \times\{0\}$ ensures that $I_{1}^{\varepsilon}$ and $I_{2}^{\varepsilon}$ tend to zero at $\partial \mathfrak{z})$.

In light of the exponential decay of Proposition 6.5 we next see that there is a $C>0$ such that for all $x \in \mathcal{N} \backslash(\partial \mathfrak{z} \cup \mathcal{C})$,

$$
\begin{aligned}
\left|I_{1}^{\varepsilon}(x)\right| & \leq C \varepsilon^{\nu-1} \varepsilon^{\nu(1 / \nu-1)}\|\phi\|_{C^{4}(\mathcal{N})} \exp \left[-C^{-1}|\mathfrak{D}(x)|^{1 / \nu} / \varepsilon\right] \\
& =C\|\phi\|_{C^{4}(\mathcal{N})} \exp \left[-C^{-1}|\mathfrak{D}(x)|^{1 / \nu} / \varepsilon\right] \\
\left|I_{2}^{\varepsilon}(x)\right| & \leq C \varepsilon^{\nu}\|\phi\|_{C^{4}(\mathcal{N})} \exp \left[-C^{-1}|\mathfrak{D}(x)|^{1 / \nu} / \varepsilon\right]
\end{aligned}
$$

for all $\varepsilon>0$. Similarly, we see that there is a constant $C>0$ such that for all $x \in \mathcal{N} \backslash(\partial \mathfrak{z} \cup \mathcal{C})$,

$$
\begin{aligned}
&\left|I_{i}^{\varepsilon}(x)\right| \leq C \varepsilon^{\nu-2} b_{n}(\mathfrak{D}(x))\|\phi\|_{C^{4}(\mathcal{N})} \exp [\left.-C^{-1}|\mathfrak{D}(x)|^{1 / \nu} / \varepsilon\right] \\
& \leq C \varepsilon^{\nu-2+\nu n}\|\phi\|_{C^{4}(\mathcal{N})} \exp \left[-C^{-1}|\mathfrak{D}(x)|^{1 / \nu} / \varepsilon\right] \\
& \leq C\|\phi\|_{C^{4}(\mathcal{N})} \exp \left[-C^{-1}|\mathfrak{D}(x)|^{1 / \nu} / \varepsilon\right]
\end{aligned}
$$

for $i \in\{3,4\}$ and $\varepsilon>0$. This gives us the first bound of (27). 
To get the second bound of (27), we compute that

$$
\begin{aligned}
\left\langle d \Psi^{\varepsilon}, d \Psi^{\varepsilon}\right\rangle(x)= & \varepsilon^{2 \nu}\left(\frac{\partial \tilde{\Psi}}{\partial \theta}\right)^{2}\left\langle d \Theta^{\circ}, d \Theta^{\circ}\right\rangle(x) \\
+ & +\nu^{-2} \varepsilon^{2 \nu-2} b_{1 / \nu-1}^{2}(\mathfrak{D}(x))\left(\frac{\partial \tilde{\Psi}}{\partial d}\right)^{2}\langle d \mathfrak{D}, d \mathfrak{D}\rangle(x) \\
& +2 \nu^{-1} \varepsilon^{\nu-1} b_{1 / \nu-1}(\mathfrak{D}(x)) \frac{\partial \tilde{\Psi}}{\partial \theta} \frac{\partial \tilde{\Psi}}{\partial d}\left\langle d \Theta^{\circ}, d \mathfrak{D}\right\rangle(x)
\end{aligned}
$$

where, as usual, $\tilde{\Psi}$ and its various derivatives are all evaluated at $\left(\Theta^{\circ}(x), b_{1 / \nu}(\mathfrak{D}(x))\right.$ $/ \varepsilon)$. Again using the exponential decay Proposition 6.5, we see that there is a constant $C>0$ such that

$$
\begin{aligned}
& \left|\varepsilon^{2 \nu}\left(\frac{\partial \tilde{\Psi}}{\partial \theta}\right)^{2}\left(\Theta^{\circ}(x), b_{1 / \nu}(\mathfrak{D}(x)) / \varepsilon\right)\left\langle d \Theta^{\circ}, d \Theta^{\circ}\right\rangle(x)\right| \\
& \leq C \varepsilon^{2 \nu}\|\phi\|_{C^{4}(\mathcal{N})}^{2} \exp \left[-C^{-1}|\mathfrak{D}(x)|^{1 / \nu} / \varepsilon\right], \\
& \left|\nu^{-2} \varepsilon^{2 \nu-2} b_{1 / \nu-1}^{2}(\mathfrak{D}(x))\left(\frac{\partial \tilde{\Psi}}{\partial d}\right)^{2}\left(\Theta^{\circ}(x), b_{1 / \nu}(\mathfrak{D}(x)) / \varepsilon\right)\langle d \mathfrak{D}, d \mathfrak{D}\rangle(x)\right| \\
& \leq C \varepsilon^{2 \nu-2+2 \nu(1 / \nu-1)}\|\phi\|_{C^{4}(\mathcal{N})}^{2} \exp \left[-C^{-1}|\mathfrak{D}(x)|^{1 / \nu} / \varepsilon\right] \\
& \leq C\|\phi\|_{C^{4}(\mathcal{N})}^{2} \exp \left[-C^{-1}|\mathfrak{D}(x)|^{1 / \nu} / \varepsilon\right] \text {, } \\
& \left|2 \nu^{-1} \varepsilon^{\nu-1} b_{1 / \nu-1}(\mathfrak{D}(x))\left(\frac{\partial \tilde{\Psi}}{\partial \theta} \frac{\partial \tilde{\Psi}}{\partial d}\right)\left(\Theta^{\circ}(x), b_{1 / \nu}(\mathfrak{D}(x)) / \varepsilon\right)\left\langle d \Theta^{\circ}, d \mathfrak{D}\right\rangle(x)\right| \\
& \leq C \varepsilon^{\nu-1+\nu(1 / \nu-1)}\|\phi\|_{C^{4}(\mathcal{N})}^{2} \exp \left[-C^{-1}|\mathfrak{D}(x)|^{1 / \nu} / \varepsilon\right] \\
& \leq C\|\phi\|_{C^{4}(\mathcal{N})}^{2} \exp \left[-C^{-1}|\mathfrak{D}(x)|^{1 / \nu} / \varepsilon\right]
\end{aligned}
$$

for all $\varepsilon>0$. This gives us the second bound; the third is clear.

Let's now return to the proof of Proposition 6.2. We would like to apply the martingale problem to $\Psi^{\varepsilon}$. There are two remaining obstacles. First, $\Psi^{\varepsilon}$ is defined on $\mathcal{N}$ rather than all of $\mathbb{R}^{2}$, and second it is not even $C^{2}$ on $\mathcal{N}$ (the second derivatives may have discontinuities on $\partial \mathfrak{z} \cup \mathcal{C}$ ). The following approximation result tells us that the continuity of $\Psi^{\varepsilon}$ is in fact sufficient.

Lemma 6.7. Suppose that $\Psi \in C^{1}\left(\mathbb{R}^{2}\right)$ and that $\Psi$ is $C^{2}$ on $\mathbb{R}^{2} \backslash(\partial \mathfrak{z} \cup \mathcal{C})$. Suppose also that $\lim _{x \in \mathbb{R}^{2} \backslash\left(\partial_{\mathfrak{z}} \cup \mathcal{C}\right)} \mathscr{L}^{\varepsilon} \Psi(y)$ exists for all $x \in \partial \mathfrak{z} \cup \mathcal{C}$. Fix $0 \leq r_{1}<r_{2}<\cdots<$ $r_{n} \leq s<t$ and $\left\{\varphi_{j} ; j=1,2 \ldots n\right\} \subset C_{b}\left(\mathbb{R}^{2}\right)$. Then for every $\varepsilon>0$,

$$
\mathbb{E}^{\varepsilon}\left[\left\{\Psi\left(X_{t \wedge \mathfrak{e}}\right)-\Psi\left(X_{s \wedge \mathfrak{e}}\right)-\int_{s \wedge \mathfrak{e}}^{t \wedge \mathfrak{e}}\left(\mathscr{L}^{\varepsilon} \Psi\right)\left(X_{u}\right) d u\right\} \prod_{j=1}^{n} \varphi_{j}\left(X_{r_{j}}\right)\right]=0 .
$$

Proof. Mollify $\Psi$.

We now can give the 
Proof of Proposition 6.2. Let's cut off $\Psi^{\varepsilon}$ near the edges of $\mathcal{N}$. To do so, let $\eta \in$ $C_{c}^{\infty}(\mathbb{R} ;[0,1])$ be such that $\eta(u)=0$ if $|u| \geq 2 \varkappa / 3$ and $\eta(u)=1$ if $|u| \leq \varkappa / 3$. Set

$$
\hat{\Psi}^{\varepsilon}(x) \stackrel{\text { def }}{=} \eta(\mathfrak{D}(x)) \Psi^{\varepsilon}(x)
$$

for all $x \in \overline{\mathbf{S}}$. Then we have that $\hat{\Psi}^{\varepsilon} \in C^{1}\left(\mathbb{R}^{2}\right), \hat{\Psi}^{\varepsilon}$ is $C^{2}$ on $\mathbb{R}^{2} \backslash(\partial \mathfrak{z} \cup \mathcal{C})$, and $\lim _{y \in \overline{\mathbb{R}}^{2} \backslash(\partial \mathfrak{z} \cup \mathcal{C})} \mathscr{L}^{\varepsilon} \hat{\Psi}^{\varepsilon}(y)$ exists for all $x \in \partial \mathfrak{z} \cup \mathcal{C}$. Furthermore, we can compute that

$$
\begin{aligned}
&\left(\mathscr{L}^{\varepsilon} \hat{\Psi}^{\varepsilon}\right)(x)- \bar{G}^{\varepsilon}(x)=\eta(\mathfrak{D}(x))\left\{\left(\mathscr{L}^{\varepsilon} \Psi^{\varepsilon}\right)(x)-\bar{G}^{\varepsilon}(x)\right\} \\
&+ \Psi^{\varepsilon}(x)\left\{\dot{\eta}(\mathfrak{D}(x))(\mathscr{L} \mathfrak{D})(x)+\frac{1}{2} \ddot{\eta}(\mathfrak{D}(x))\langle d \mathfrak{D}, d \mathfrak{D}\rangle(x)\right\} \\
&+\dot{\eta}(\mathfrak{D}(x))\left\langle d \Psi^{\varepsilon}, d \mathfrak{D}\right\rangle(x)
\end{aligned}
$$

for all $x \in \mathbb{R}^{2} \backslash(\partial \mathfrak{z} \cup \mathcal{C})$. Hence there is a $C>0$ such that

$$
\begin{aligned}
&\left|\mathscr{L}^{\varepsilon} \hat{\Psi}^{\varepsilon}(x)-\bar{G}^{\varepsilon}(x)\right| \\
& \leq C\|\phi\|_{C^{4}(\mathcal{N})}\left\{\varepsilon^{\nu}+\exp [\right.\left.\left.-C^{-1}|\mathfrak{D}(x)|^{1 / \nu} / \varepsilon\right] \chi_{[\varkappa / 3,2 \varkappa / 3]}(\mathfrak{D}(x))\right\} \\
& \leq C\|\phi\|_{C^{4}(\mathcal{N})}\left\{\varepsilon^{\nu}+\exp \left[-C^{-1}(\varkappa / 3)^{1 / \nu} / \varepsilon\right]\right\}
\end{aligned}
$$

for all $\varepsilon>0$ and $x \in \mathcal{N} \backslash(\partial \mathfrak{z} \cup \mathcal{C})$. We now use Lemma 6.7 and the ideas of Remark 6.4. We see that there is a $C>0$ (which does not depend on $\phi$ ) such that

$$
\begin{array}{r}
\left|\mathbb{E}^{\varepsilon}\left[\int_{s \wedge \mathfrak{e}}^{t \wedge \mathfrak{e}} \bar{G}^{\varepsilon}\left(X_{u}\right) d u \prod_{j=1}^{n} \varphi_{j}\left(X_{r_{j}}\right)\right]\right| \\
\leq C\|\phi\|_{C^{4}\left(\mathbb{R}^{2}\right)}\left\{\varepsilon^{\nu}+\mathbb{E}^{\varepsilon}\left[\int_{s \wedge \mathfrak{e}}^{t \wedge \mathfrak{e}}\left\{\varepsilon^{\nu}+e^{-C^{-1} / \varepsilon}\right\} d u\right]\right\}
\end{array}
$$

for all $\varepsilon>0$. This proves the result.

\section{Proof of Main Steps}

Let's first prove Proposition 4.12 .

Proof of Proposition 4.19. Define

$$
\begin{aligned}
\omega_{K}(\varepsilon) \stackrel{\text { def }}{=} \sup _{\substack{x \in \overline{\mathbf{S}} \\
\varepsilon^{\nu} \leq \mathfrak{D}(x) \leq 2 \varepsilon^{\nu}}}\left|\tilde{f}_{1}^{\varepsilon}(x)-\tilde{f}_{2}^{\varepsilon}(x)\right| \\
\leq \sup _{\substack{x \in \overline{\mathbf{S}} \\
\varepsilon^{\nu} \leq \mathfrak{D}(x) \leq 2 \varepsilon^{\nu}}}\left\{\left|\tilde{f}_{\mathfrak{z}}^{\varepsilon}(x)-f(\star)\right|+\left|f_{\Gamma}^{\varepsilon}(\mathfrak{D}(x))-f(\star)\right|\right\} .
\end{aligned}
$$

We now use the bounds on the derivatives of $\tilde{f}_{\mathfrak{z}}^{\varepsilon}$ and $\tilde{f}_{\Gamma}^{\varepsilon}$ given in Lemmas 4.5] and 4.6. We find that for some constant $C>0, \omega_{K}(\varepsilon) \leq C \varepsilon^{\nu-\nu^{\prime}}$ for all $\varepsilon>0$. Thun 1 $\lim _{\varepsilon \rightarrow 0} \omega_{K}(\varepsilon)=0$ and hence for some constant $C>0$,

$$
\mathbb{E}^{\varepsilon}\left[\left|\int_{0}^{t \wedge \mathfrak{e}} K^{\varepsilon}\left(X_{s}\right) d s\right|\right] \leq C \omega_{K}(\varepsilon) \mathbb{E}^{\varepsilon}\left[\int_{0}^{t \wedge \mathfrak{e}} \frac{1}{\varepsilon^{\nu}} \chi_{[1,2]}\left(\mathfrak{D}\left(X_{s}\right) / \varepsilon^{\nu}\right) d s\right]
$$

\footnotetext{
${ }^{1}$ We use here the fact that $\nu^{\prime}<\nu=\frac{2}{n+1}$.
} 

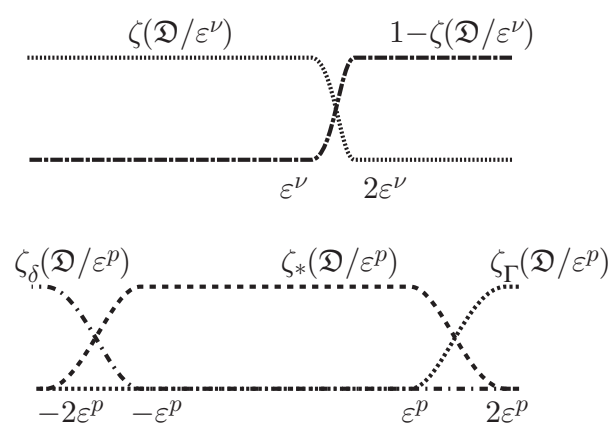

Figure 4. Cutoff Functions for Proof of Proposition 4.10

which, by Proposition 5.1, gives the desired result.

Next, we give the

Proof of Proposition 4.10. Let $\zeta_{\mathfrak{z}}, \zeta_{\star}$ and $\zeta_{\Gamma}$ be a partition of unity such that $\operatorname{supp} \zeta_{\mathfrak{z}} \subset(-\infty,-1), \operatorname{supp} \zeta_{\star} \subset(-2,2)$, and $\operatorname{supp} \zeta_{\Gamma} \subset(1, \infty)$. Fix next $p$ such that

$$
2 \nu^{\prime}<p<\min \left\{\nu, \frac{2-4 \nu^{\prime}}{n+1}\right\}
$$

our choice of $\nu$ and $\nu^{\prime}$ ensure that we can do this. Keep in mind the statements of Lemmas 4.5 and 4.6 See also Figure 4.

Step 1. Since $\varepsilon^{p} \gg \varepsilon^{\nu}$, we hav $\oint^{3}$ that

$$
L^{\varepsilon}(x) \zeta_{\mathfrak{z}}\left(\mathfrak{D}(x) / \varepsilon^{p}\right)=\left(\mathscr{L} \tilde{f}_{\mathfrak{z}}^{\varepsilon}\right)(x) \zeta_{\mathfrak{z}}\left(\mathfrak{D}(x) / \varepsilon^{p}\right)
$$

for all $x \in \overline{\mathbf{S}}$. Thus since the support of $\zeta_{\mathfrak{z}}\left(\mathfrak{D} / \varepsilon^{p}\right)$ is contained in $\mathfrak{z}^{\circ}$,

$$
\begin{aligned}
\varlimsup_{\varepsilon \rightarrow 0} \mathbb{E}^{\varepsilon}\left[\left|\int_{0}^{t \wedge \mathfrak{e}}\left\{L^{\varepsilon}\left(X_{s}\right)-\left(\mathscr{L}^{\dagger} f\right)\left(Y_{s}\right)\right\} \zeta_{\mathfrak{z}}\left(\mathfrak{D}\left(X_{s}\right) / \varepsilon^{p}\right) d s\right|\right] \\
\leq \varlimsup_{\varepsilon \rightarrow 0}\left\|\mathscr{L} \tilde{f}^{\varepsilon}-\mathscr{L}^{\dagger} f\right\|_{C\left(\mathfrak{z}^{\circ}\right)}=0 .
\end{aligned}
$$

Step 2. It is clear that

$$
\sup _{\varepsilon>0} \varepsilon^{2 \nu^{\prime}} \sup _{\substack{x \in \overline{\mathbf{S}} \\-2 \varepsilon^{p} \leq \mathfrak{D}(x) \leq 2 \varepsilon^{p}}}\left|L^{\varepsilon}(x)\right|<\infty .
$$

\footnotetext{
${ }^{2}$ In order for such a $p$ to exist, we need that

$$
\nu^{\prime}<\min \left\{\frac{\nu}{2}, \frac{1}{n+3}\right\}=\frac{1}{n+3} .
$$
}

${ }^{3}$ We use here the fact that $p<\nu$. 
Thus there is a $C>0$ such that

$$
\begin{aligned}
\varlimsup_{\varepsilon \rightarrow 0} \mathbb{E}^{\varepsilon}\left[\left|\int_{0}^{t \wedge \mathfrak{e}}\left\{L^{\varepsilon}\left(X_{s}\right)-\left(\mathscr{L}^{\dagger} f\right)\left(Y_{s}\right)\right\} \zeta_{\star}\left(\mathfrak{D}\left(X_{s}\right) / \varepsilon^{p}\right) d s\right|\right] \\
\leq \varlimsup_{\varepsilon \rightarrow 0} C \varepsilon^{-2 \nu^{\prime}} \mathbb{E}^{\varepsilon}\left[\int_{0}^{t \wedge \mathfrak{e}} \chi_{[-2,2]}\left(\mathfrak{D}\left(X_{s}\right) / \varepsilon^{p}\right) d s\right]=0 \\
\leq \varlimsup_{\varepsilon \rightarrow 0} C \varepsilon^{p-2 \nu^{\prime}} \mathbb{E}^{\varepsilon}\left[\int_{0}^{t \wedge \mathfrak{e}} \frac{1}{\varepsilon^{p}} \chi_{[-2,2]}\left(\mathfrak{D}\left(X_{s}\right) / \varepsilon^{p}\right) d s\right]=0,
\end{aligned}
$$

the last equality following from our choict 4 of $p$.

Step 3. Note next that by Lemma 4.6 there is a constant $C>0$ such that

$$
\left\|\mathscr{L} \tilde{f}_{\Gamma}^{\varepsilon}\right\|_{C^{2}(\overline{\mathbf{S}} \backslash \mathfrak{z})} \leq C \varepsilon^{-4 \nu^{\prime}}
$$

for all $\varepsilon>0$ (note that we are taking the $C^{2}$ norm of $\mathscr{L} \tilde{f}_{\Gamma}^{\varepsilon}$ ). Thus by Lemma 6.1 there is a $C>0$ such that

$$
\begin{aligned}
& \mathbb{E}^{\varepsilon}\left[\mid \int_{0}^{t \wedge \mathfrak{e}}\left\{L^{\varepsilon}\left(X_{s}\right)-\left(\mathscr{L}^{\dagger} f\right)\right.\right.\left.\left.\left(Y_{s}\right)\right\} \zeta_{\Gamma}\left(\mathfrak{D}\left(X_{s}\right) / \varepsilon^{p}\right) d s \mid\right] \\
& \leq C \varepsilon^{2-p(n+1)-4 \nu^{\prime}}+\left\|\mathscr{L}_{D} f_{\Gamma}^{\varepsilon}-\mathscr{L}_{D} f_{D}\right\|_{C((0, \bar{D}))} .
\end{aligned}
$$

By our choice of $p$, this last term tends to zerd5. Combining (31), 32) and (33), we have the desired result.

Now let's prove the part of Proposition 4.11 corresponding to $G_{1}^{\varepsilon}$ and $G_{2}^{\varepsilon}$.

Lemma 7.1. Fix times $0 \leq r_{1}<r_{2}<\cdots<r_{n} \leq s<t$ and test functions $\left\{\varphi_{j} ; j=1,2 \ldots n\right\} \subset C_{b}\left(\mathbb{R}^{2}\right)$. Then for $i \in\{1,2\}$,

$$
\lim _{\varepsilon \rightarrow 0} \mathbb{E}^{\varepsilon}\left[\int_{s \wedge \mathfrak{e}}^{t \wedge \mathfrak{e}} G_{i}^{\varepsilon}\left(X_{u}\right) d u \prod_{j=1}^{n} \varphi_{j}\left(X_{r_{j}}\right)\right]=0 .
$$

Proof. For each $\varepsilon>0$, let $\phi_{1}^{\varepsilon}$ and $\phi_{2}^{\varepsilon}$ in $C^{4}\left(\mathbb{R}^{2}\right)$ be such that

$$
\begin{aligned}
& \phi_{1}^{\varepsilon}(x) \stackrel{\text { def }}{=}\left\langle d \tilde{f}_{\mathfrak{z}}^{\varepsilon}, d \mathfrak{D}\right\rangle(x)-\left\langle d \tilde{f}_{\Gamma}^{\varepsilon}, d \mathfrak{D}\right\rangle(x), \\
& \phi_{2}^{\varepsilon}(x) \stackrel{\text { def }}{=} \frac{\tilde{f}_{\mathfrak{z}}^{\varepsilon}(x)-\tilde{f}_{\Gamma}^{\varepsilon}(x)}{\mathfrak{D}(x)}\langle d \mathfrak{D}, d \mathfrak{D}\rangle(x)
\end{aligned}
$$

for all $x \in \overline{\mathbf{S}}$ such that $\varepsilon^{\nu}<\mathfrak{D}(x)<2 \varepsilon^{\nu}$. Lemmas 9.8 and 9.9 give us a $C>0$ such that

$$
\left\|\phi_{i}^{\varepsilon}\right\|_{C^{4}(\overline{\mathbf{S}})} \leq C \varepsilon^{-6 \nu^{\prime}}
$$

for $i \in\{1,2\}$ and $\varepsilon>0$. Let's also define

$$
\begin{aligned}
& \varpi_{1}(\vartheta) \stackrel{\text { def }}{=}-\frac{\dot{\zeta}(\vartheta)}{\vartheta^{n}}, \\
& \varpi_{2}(\vartheta) \stackrel{\text { def }}{=}-\frac{\ddot{\zeta}(\vartheta)}{2 \vartheta^{n-1}},
\end{aligned}
$$

${ }^{4}$ We use here that $p>2 \nu^{\prime}$.

${ }^{5}$ We use here that $2-4 \nu^{\prime}-p(n+1)>0$. The combination of this and footnotes 3 and 4 gives (30). 
and observe that for all $x \in \overline{\mathbf{S}}$

$$
\begin{array}{r}
-\varepsilon^{-\nu} \dot{\zeta}\left(\mathfrak{D}(x) / \varepsilon^{\nu}\right)=\varepsilon^{-2} \varpi_{1}\left(\mathfrak{D}(x) / \varepsilon^{\nu}\right) b_{n}(\mathfrak{D}(x)), \\
-\frac{1}{2} \varepsilon^{-2 \nu} \mathfrak{D}(x) \ddot{\zeta}\left(\mathfrak{D}(x) / \varepsilon^{\nu}\right)=\varepsilon^{-2} \varpi_{2}\left(\mathfrak{D}(x) / \varepsilon^{\nu}\right) b_{n}(\mathfrak{D}(x))
\end{array}
$$

and thus

$$
\begin{aligned}
G_{1}^{\varepsilon}(x) & =\varepsilon^{-2} \varpi_{1}\left(\mathfrak{D}(x) / \varepsilon^{\nu}\right) b_{n}(\mathfrak{D}(x)) \phi_{1}^{\varepsilon}(x), \\
G_{2}^{\varepsilon}(x) & =\varepsilon^{-2} \varpi_{2}\left(\mathfrak{D}(x) / \varepsilon^{\nu}\right) b_{n}(\mathfrak{D}(x)) \phi_{2}^{\varepsilon}(x) .
\end{aligned}
$$

Thus, by Proposition 6.2 and the triangle inequality, there is a $C>0$ such that

$$
\begin{aligned}
& \left|\mathbb{E}^{\varepsilon}\left[\int_{s \wedge \mathfrak{e}}^{t \wedge \mathfrak{e}} G_{i}^{\varepsilon}\left(X_{u}\right) d u \prod_{j=1}^{n} \varphi_{j}\left(X_{r_{j}}\right)\right]\right| \leq C(1+t) \varepsilon^{\nu-6 \nu^{\prime}} \\
& +\varepsilon^{-2}\left|\mathbb{E}^{\varepsilon}\left[\int_{s^{\wedge} \mathfrak{e}}^{t \wedge \mathfrak{e}} b_{n}\left(\mathfrak{D}\left(X_{u}\right)\right)\left|\varpi_{i}\left(\mathfrak{D}\left(X_{u}\right) / \varepsilon^{\nu}\right)\left(\mathbf{A}^{D} \phi_{i}^{\varepsilon}\right)\left(\mathfrak{D}\left(X_{u}^{\varepsilon}\right)\right)\right| d u \prod_{j=1}^{n} \varphi_{j}\left(X_{r_{j}}\right)\right]\right|
\end{aligned}
$$

for $\varepsilon>0$ and $i \in\{1,2\}$. Now define

$$
\omega_{i}(\varepsilon) \stackrel{\text { def }}{=} \sup _{\varepsilon^{\nu}<d<2 \varepsilon^{\nu}}\left|\left(\mathbf{A}^{D} \phi_{i}^{\varepsilon}\right)(d)\right| ;
$$

Lemmas 9.8 and 9.9 in Section 9 ensure that (due to the glueing conditions)

$$
\lim _{\varepsilon \rightarrow 0} \omega_{i}(\varepsilon)=0
$$

for $i \in\{1,2\}$. Thus by calculations similar to that of (23), we get that there is a constant $C>0$ such that

$$
\begin{aligned}
& \varepsilon^{-2}\left|\mathbb{E}^{\varepsilon}\left[\int_{s^{\wedge} \mathfrak{e}}^{t \wedge \mathfrak{e}} b_{n}\left(\mathfrak{D}\left(X_{u}\right)\right)\left|\varpi_{i}\left(\mathfrak{D}\left(X_{u}\right) / \varepsilon^{\nu}\right)\left(\mathbf{A}^{D} \phi_{i}^{\varepsilon}\right)\left(\mathfrak{D}\left(X_{u}^{\varepsilon}\right)\right)\right| d u \prod_{j=1}^{n} \varphi_{j}\left(X_{r_{j}}\right)\right]\right| \\
& \leq C \omega_{i}(\varepsilon) \text {. }
\end{aligned}
$$

Combine this, (35) and (37) to get the stated result; we here use our choice $e^{6}$ of $\nu$ and $\nu^{\prime}$.

Finally, we prove the result for $G_{3}^{\varepsilon}$.

Lemma 7.2. Fix times $0 \leq r_{1}<r_{2}<\cdots<r_{n} \leq s<t$, and test functions $\left\{\varphi_{j} ; j=1,2 \ldots n\right\} \subset C_{b}\left(\mathbb{R}^{2}\right)$. Then

$$
\lim _{\varepsilon \rightarrow 0} \mathbb{E}^{\varepsilon}\left[\int_{s \wedge \mathfrak{e}}^{t \wedge \mathfrak{e}} G_{3}^{\varepsilon}\left(X_{u}\right) d u \prod_{j=1}^{n} \varphi_{j}\left(X_{r_{j}}\right)\right]=0 .
$$

Proof. Note first that

$$
\mathbf{A}^{D}\left(\bar{\nabla} \mathfrak{D}, \nabla \tilde{f}_{\mathfrak{z}}^{\varepsilon}\right)_{T \mathbb{R}^{2}} \equiv 0 .
$$

Lemma 9.10 tells us that we can find a $\phi_{3}^{\varepsilon} \in C^{4}\left(\mathbb{R}^{2}\right)$ such that

$$
\left(\bar{\nabla} \mathfrak{D}, \nabla \tilde{f}_{\mathfrak{z}}^{\varepsilon}\right)_{T \mathbb{R}^{2}}(x)=\mathfrak{D}(x) \phi_{3}^{\varepsilon}(x)
$$

${ }^{6}$ We need here that $\nu^{\prime}<\frac{\nu}{6}=\frac{1}{3(n+1)}$. 
for all $x \in \mathcal{N}$ and such that $\mathbf{A}^{D} \phi_{3}^{\varepsilon} \equiv 0$. Furthermore, it gives us a $C>0$ such that $\left\|\phi_{3}^{\varepsilon}\right\|_{C^{4}(\mathcal{N})} \leq C \varepsilon^{-7 \nu^{\prime}}$ for all $\varepsilon>0$. Define

$$
\varpi_{3}(\vartheta) \stackrel{\text { def }}{=} n\{1-\zeta(\vartheta)\}, \quad \vartheta \in \mathbb{R},
$$

then we can write

$$
G_{3}^{\varepsilon}(x)=\varepsilon^{-2} b_{n}(\mathfrak{D}(x)) \phi_{3}^{\varepsilon}(x) \varpi_{3}\left(\mathfrak{D}(x) / \varepsilon^{\nu}\right)
$$

for all $x \in \overline{\mathbf{S}}$. By Proposition 6.2 and our choice of $\nu$ and $\nu / 7$, we thus get the result.

\section{UNIQUENESS}

The only remaining issue is uniqueness; we would like to know that there is only one limit point of the $\mathbb{P}^{\varepsilon, \dagger}$ 's. We shall prove

Proposition 8.1. The operator $\mathscr{L}^{\dagger}$ generates a strongly continuous, positive, contraction semigroup on $C(\mathfrak{M})$.

As usual, we endow $C(\mathfrak{M})$ with the topology generated by the $\|\cdot\|_{C(\mathfrak{M})}$ norm. According to [5, Theorem 4.2.2], we need to prove three things: that $\mathscr{D}^{\dagger}$ is dense in $C(\mathfrak{M})$, that $\mathscr{L}^{\dagger}$ satisfies the positive maximum principle, and that the range of $\lambda-\mathscr{L}^{\dagger}$ is dense in $C(\mathfrak{M})$ for some $\lambda>0$. We begin with

Lemma 8.2. The set $\mathscr{D}^{\dagger}$ is dense in $C(\mathfrak{M})$.

Proof. Fix $f \in C(\mathfrak{M})$. We will first kill the variation of $f$ near $\star$ and $\circledast$. Let $\zeta \in C^{\infty}(\mathbb{R})$ be nonnegative, have support in $[-1,1]$, and be such that $\zeta(\vartheta)=1$ when $|\vartheta| \leq 1 / 2$. For each $n$, define

$$
\begin{aligned}
f_{n}([x]) & \stackrel{\text { def }}{=} f([x])\{1-\zeta(n \mathfrak{D}(x))-\zeta(n(\mathfrak{D}(x)-\bar{D}))\} \\
& +f(\star) \zeta(n \mathfrak{D}(x))+f(\circledast) \zeta(n(\mathfrak{D}(x)-\bar{D})), \quad x \in \overline{\mathbf{S}} .
\end{aligned}
$$

It is easy to see that

$$
\lim _{n \rightarrow \infty}\left\|f_{n}-f\right\|_{C(\mathfrak{M})}=0 .
$$

For each $n$, now let $f_{n}^{\prime} \in C^{\infty}\left(\mathfrak{z}^{\circ} \cup \Gamma\right)$ be such that

$$
\sup _{\substack{x \in \mathbf{S} \\ \mathcal{D}(x)|\geq 1 /(2 n) \\(x)-\bar{D}| \geq 1 /(2 n)}}\left|f([x])-f_{n}^{\prime}([x])\right| \leq \frac{1}{n}
$$

(the $f_{n}^{\prime}$ 's can be constructed via standard mollification procedures). Define

$$
\begin{aligned}
\tilde{f}_{n}([x]) \stackrel{\text { def }}{=} f_{n}^{\prime}([x])\{1-\zeta(n \mathfrak{D}(x))-\zeta(n(\mathfrak{D}(x)-\bar{D}))\} \\
+f(\star) \zeta(n \mathfrak{D}(x))+f(\circledast) \zeta(n(\mathfrak{D}(x)-\bar{D})), \quad x \in \overline{\mathbf{S}},
\end{aligned}
$$

then

$$
\lim _{n \rightarrow \infty}\left\|f_{n}-\tilde{f}_{n}\right\|_{C(\mathfrak{M})}=0
$$

7 We use here that

$$
\nu^{\prime}<\frac{\nu}{7}=\frac{2}{7(n+1)}
$$


This and (39) imply that

$$
\lim _{n \rightarrow \infty}\left\|\tilde{f}_{n}-f\right\|_{C(\mathfrak{M})}=0 .
$$

Each of the $\tilde{f}_{n}$ 's is in $C^{\infty}\left(\mathfrak{z}^{\circ} \cup \Gamma\right)$ and each of them is constant in a small neighborhood of $\star$ and $\circledast$; they are thus clearly in $\mathscr{D}^{\dagger}$.

The next step is to verify that $\mathscr{L}^{\dagger}$ satisfies the positive maximum principle.

Lemma 8.3. The operator $\mathscr{L}^{\dagger}$ satisfies the positive maximum principle; i.e., for $f \in \mathscr{D}^{\dagger}$ and $x^{*} \in \mathfrak{M}$ such that $\sup _{x \in \mathfrak{M}} f(x)=f\left(x^{*}\right) \geq 0$, we have that $\left(\mathscr{L}^{\dagger} f\right)\left(x^{*}\right) \leq$ 0 .

Proof. If $x^{*}=\circledast$, then by definition of $\mathscr{D}^{\dagger},\left(\mathscr{L}^{\dagger} f\right)\left(x^{*}\right)=0$. Assume next that $x^{*} \in \mathfrak{z}^{\circ} \cup \Gamma$. Since $\mathscr{L}^{\dagger}$ is local and can be represented by a strongly elliptic operator on $\mathfrak{z}^{\circ}$ and $\Gamma$, we must here too have that $\left(\mathscr{L}^{\dagger} f\right)\left(x^{*}\right) \leq 0$. Finally, assume that $x^{*}=\star$. By assumption that $f$ attains its maximum at $x^{*}=\star$, we have that $\mathscr{G}_{\text {inner }} f \geq 0$ and $\mathscr{G}_{\text {outer }} f \leq 0$ (use Lemmas 4.4 and 9.5 for $\mathscr{G}_{\text {outer }} f$ and use Lemma 9.11 for $\mathscr{G}_{\text {inner }}$ ); hence $\mathscr{G}_{\text {inner }} f=\mathscr{G}_{\text {outer }} f=0$ and thus (using Lemma 9.5) $\dot{f}_{D}(0)=$ $\mathscr{G}_{\text {outer }} f / \sigma_{D}^{2}(0)=0$. Since we have assumed that $\star$ is a local maximum of $f$ and $f_{D} \in C^{2}([0, \bar{D}])$ (Lemma 4.4), we thus have that $\ddot{f}_{D}(0) \leq 0$. Finally, we have that

$$
\left(\mathscr{L}^{\dagger} f\right)(\star)=\lim _{d \searrow 0}\left(\mathscr{L}_{D} f_{D}\right)(d)=\frac{1}{2} \sigma_{D}^{2}(0) \ddot{f}_{D}(0)+b_{D}(0) \dot{f}_{D}(0) \leq 0 .
$$

This completes the proof.

Finally, we prove

Lemma 8.4. The range of $\lambda-\mathscr{L}^{\dagger}$ is dense in $C(\mathfrak{M})$ for some $\lambda>0$.

Proof. It suffices to fix a $\varphi \in C^{\infty}(\mathfrak{M})$ and a $\lambda>0$ and find an $f \in \mathscr{D}^{\dagger}$ such that

$$
\left(\mathscr{L}^{\dagger} f\right)(x)-\lambda f(x)=\varphi(x), \quad x \in \mathfrak{M}
$$

We separately consider several PDE's on $\mathfrak{z}^{\circ}$ (a domain in $\mathbb{R}^{2}$ ) and on $\Gamma$ (diffeomorphic to $(0,1))$. Let $f_{\mathfrak{z}}$ and $g_{\mathfrak{z}}$ be weak solutions of the PDE's

$$
\begin{aligned}
\mathscr{L} f_{\mathfrak{z}}-\lambda f_{\mathfrak{z}} & =\varphi & & \text { on } \mathfrak{z}^{\circ}, \\
f_{\mathfrak{z}} & =0 & & \text { on } \partial \mathfrak{z},
\end{aligned}
$$

and

$$
\begin{aligned}
\mathscr{L} g_{\mathfrak{z}}-\lambda g_{\mathfrak{z}}=0 & & \text { on } \mathfrak{z}^{\circ}, \\
g_{\mathfrak{z}}=1 & & \text { on } \partial \mathfrak{z},
\end{aligned}
$$

and let $f_{\Gamma}, g_{\star, \Gamma}$, and $g_{\circledast, \Gamma}$ be weak solutions of the PDE's

$$
\begin{aligned}
\mathscr{L} f_{\Gamma}-\lambda f_{\Gamma} & =\varphi_{D} \quad \text { on }(0, \bar{D}), \\
f_{\Gamma}(0) & =0 \\
f_{\Gamma}(\bar{D}) & =0
\end{aligned}
$$

and

$$
\begin{aligned}
\mathscr{L} g_{\star, \Gamma}-\lambda g_{\star, \Gamma} & =0 \quad \text { on }(0, \bar{D}), \\
g_{\star, \Gamma}(0) & =1 \\
g_{\star, \Gamma}(\bar{D}) & =0
\end{aligned}
$$


and

$$
\begin{aligned}
\mathscr{L} g_{\circledast, \Gamma}-\lambda g_{\circledast, \Gamma} & =0 \quad \text { on }(0, \bar{D}), \\
g_{\circledast, \Gamma}(0) & =0, \\
g_{\circledast, \Gamma}(\bar{D}) & =1 .
\end{aligned}
$$

By standard results [6] Theorem 6.2.5], all of these PDE's have solutions (to directly apply the results of [6. Theorem 6.2.5], one may first subtract off smooth functions which satisfy the boundary data); furthermore, by standard smoothness results [6. Theorem 6.3.6], these solutions are infinitely smooth. We will find a solution $f$ of (40) of the form

$$
\tilde{f}([x])= \begin{cases}f_{\mathfrak{z}}(x)+C_{\star} g_{\mathfrak{z}}(x) & \text { if } x \in \mathfrak{z}^{\circ}, \\ C_{\star} & \text { if } x \in \partial \mathfrak{z}, \\ f_{\Gamma}(\mathfrak{D}(x))+C_{\star} g_{\star, \Gamma}(\mathfrak{D}(x))+C_{\circledast} g_{\circledast, \Gamma}(\mathfrak{D}(x)) & \text { if } x \in \overline{\mathbf{S}} \backslash \mathfrak{z}\end{cases}
$$

for some choice of $C_{\star}$ and $C_{\circledast}$. First, note that $\tilde{f} \in C(\mathfrak{M})$ no matter what the choice of $C_{\star}$ and $C_{\circledast}$. Secondly, $\tilde{f} \in C^{2}\left(\mathfrak{z}^{\circ} \cup \Gamma\right)$, and by virtue of the above PDE's and the continuity of $\varphi$ and $\tilde{f}$, we know that $\mathscr{L}(\tilde{f} \circ \pi) \in \mathscr{D}_{\mathbf{A}}$ (unravel the notation). Thirdly, we calculate that

$$
\left(\mathscr{L}_{D} \tilde{f}_{D}\right)(\bar{D})=\lambda \tilde{f}_{D}(\bar{D})+\varphi_{D}(\bar{D})=\lambda C_{\circledast}+\varphi_{D}(\bar{D})
$$

to ensure that $\lim _{\substack{[x] \rightarrow \operatorname{la}^{\circ} \cup \Gamma \\[x] \in \mathfrak{z}^{\circ}}}\left(\mathscr{L}_{\text {ave }} \tilde{f}\right)([x])=0$, we thus need that $C_{\circledast}=-\frac{\varphi_{D}(\bar{D})}{\lambda}$. Finally, the glueing conditions require that

$$
\mathscr{G}_{\text {inner }} f_{\mathfrak{z}}+C_{\star} \mathscr{G}_{\text {inner }} g_{\mathfrak{z}}=\mathscr{G}_{\text {outer }} f_{\Gamma}+C_{\star} \mathscr{G}_{\text {outer }} g_{\star, \Gamma}-\frac{\varphi_{D}(\bar{D})}{\lambda} \mathscr{G}_{\text {outer }} g_{\circledast, \Gamma}
$$

Thus we want to take

$$
C_{\star}=\frac{\mathscr{G}_{\text {outer }} f_{\Gamma}-\frac{\varphi_{D}(\bar{D})}{\lambda} \mathscr{G}_{\text {outer }} g_{\circledast, \Gamma}-\mathscr{G}_{\text {inner }} f_{\mathfrak{z}}}{\mathscr{G}_{\text {inner }} g_{\mathfrak{z}}-\mathscr{G}_{\text {outer }} g_{\star}, \Gamma},
$$

but we need to make sure that $\mathscr{G}_{\text {inner }} g_{\mathfrak{z}} \neq \mathscr{G}_{\text {outer }} g_{\star, \Gamma}$. To do so, consider the function

$$
g([x])= \begin{cases}g_{\mathfrak{z}}(x) & \text { if } x \in \mathfrak{z}^{\circ} \\ 1 & \text { if } x \in \partial \mathfrak{z} \\ g_{\star, \Gamma}(\mathfrak{D}(x)) & \text { if } x \in \overline{\mathbf{S}} \backslash \mathfrak{z} .\end{cases}
$$

If $\mathscr{G}_{\text {inner }} g_{\mathfrak{z}}=\mathscr{G}_{\text {outer }} g_{\star, \Gamma}$, then we have constructed a nonzero element of $\mathscr{D}^{\dagger}$ such that $\mathscr{L}^{\dagger} g-\lambda g \equiv 0$ (it is easily checked that $g \in \mathscr{D}^{\dagger}$ ). Since $\mathscr{L}^{\dagger}$ obeys the positive maximum principle, it is dissipative [5. Lemma 4.2.1], and so

$$
0=\left\|\mathscr{L}^{\dagger} g-\lambda g\right\|_{C(\mathfrak{M})} \geq \lambda\|g\|_{C(\mathfrak{M})},
$$

so in fact $g \equiv 0$. Thus we cannot have that $\mathscr{G}_{\text {inner }} g_{\mathfrak{z}}=\mathscr{G}_{\text {outer }} g_{\star, \Gamma}$, so (41) makes sense.

We have

Proof of Proposition 8.1. Use the above results and [5], Theorem 4.2.2]. 


\section{Behavior of Test Functions}

Here we prove various results about the test function $f$ (which is assumed to be in $\mathscr{D}^{\dagger}$ ). We also prove the approximation results of Lemmas 4.5 and 4.6 and give some relevant results about these approximations. Thirdly, we prove various necessary results about the glueing operators $\mathscr{G}_{\text {inner }}$ and $\mathscr{G}_{\text {outer }}$. This section will rely fairly heavily upon some classical PDE theory, for which we refer the reader to [6. The harder part of our analysis will be on the 2 -dimensional domain $\mathfrak{z}^{\circ}$; in interest of keeping our arguments as parallel as possible, we will frame our simpler 1-dimensional analysis on $[0, \bar{D}]$ in the analogous ways.

We begin with a standard result.

Lemma 9.1. Fix $g \in C(\mathfrak{z})$ and a constant $\kappa \in \mathbb{R}$. Then there is a unique solution $u_{g, \kappa} \in H^{2}\left(\mathfrak{z}^{\circ}\right) \cap C(\mathfrak{z})$ of the PDE

$$
\begin{aligned}
\mathscr{L} u_{g, \kappa}=g & & \text { in } \mathfrak{z}^{\circ}, \\
u_{g, \kappa}=\kappa & & \text { on } \partial \mathfrak{z} .
\end{aligned}
$$

Fix next a nonnegative integer $k \geq 0$. If $g \in C^{k}(\mathfrak{z})$, then $u_{g, \kappa} \in C^{k}(\mathfrak{z})$, and there is a constant $C_{k}>0$ such that

$$
\begin{aligned}
\left\|u_{g, \kappa}\right\|_{H^{k+2}\left(\mathfrak{z}^{\circ}\right)} & \leq C_{k}\left\{\|g\|_{C^{k}\left(\mathfrak{z}^{\circ}\right)}+|\kappa|\right\}, \\
\left\|u_{g, \kappa}\right\|_{C^{k}(\mathfrak{z})} & \leq C_{k}\left\{\|g\|_{C^{k}(\mathfrak{z})}+|\kappa|\right\}
\end{aligned}
$$

for all $g \in C^{k}(\mathfrak{z})$ and $\kappa \in \mathbb{R}$.

Proof. Step 1. We first of all claim that there is a unique $u \in H_{0}^{1}\left(\mathfrak{z}^{\circ}\right)$ which weakly satisfies the PDE

$$
\begin{aligned}
\mathscr{L} u=g & \text { in } \mathfrak{z}^{\circ}, \\
u=0 & \text { on } \partial \mathfrak{z} .
\end{aligned}
$$

To do so, we use a standard application of the Fredholm alternative. Assume that we have a weak solution $v \in H_{0}^{1}\left(\mathfrak{z}^{\circ}\right)$ of the PDE

$$
\begin{aligned}
\mathscr{L} v & =0 & & \text { in } \mathfrak{z}^{\circ}, \\
v & =0 & & \text { on } \partial \mathfrak{z} .
\end{aligned}
$$

Since $\mathfrak{z}$ and the coefficients of $\mathscr{L}$ are all $C^{\infty}$, then [6, Theorem 6.3.6] $v \in C^{\infty}(\mathfrak{z})$. Thus, by the maximum principle [6, Theorem 6.4.1], we know that $v \equiv 0$. Hence, by the Fredholm alternative [6, Theorem 6.2.4] (and since $g \in C(\mathfrak{z}) \subset L^{2}(\mathfrak{z})$ ) a unique weak solution of (44) exists.

Step 2. By standard regularity calculations [6] Theorem 6.3.4], we then have that in fact $u \in H^{2}\left(\mathfrak{z}^{\circ}\right)$ and hence by standard Sobolev theory [6, Theorem 5.6.6] that $u \in C(\mathfrak{z})$.

Step 3. Define now $u_{g, \kappa} \stackrel{\text { def }}{=} u+\kappa$. Clearly this satisfies the stated PDE. To show uniqueness, let $u_{g, \kappa}^{\prime} \in H^{2}\left(\mathfrak{z}^{\circ}\right) \cap C(\mathfrak{z})$ be any other solution of (42), and define $\tilde{u} \stackrel{\text { def }}{=} u_{g, \kappa}^{\prime}-u_{g, \kappa}$ on $\mathfrak{z}$. Then $\mathscr{L} \tilde{u}=0$ in $\mathfrak{z}^{\circ}$, and thus $\tilde{u} \in C^{\infty}\left(\mathfrak{z}^{\circ}\right)$ [6], Theorem 6.3.3]. Since $u_{g, \kappa}^{\prime} \in C(\mathfrak{z})$, we also have that $\tilde{u} \in C(\mathfrak{z})$. Thus, by the maximum principle [6] Theorem 6.4.1], we know that in fact $\tilde{u} \equiv 0$.

Step 4. Assume now that $g \in C^{k}(\mathfrak{z})$. Then $g \in H^{k}\left(\mathfrak{z}^{\circ}\right)$ and by standard regularity calculations [6. Theorems 5.6.6 and 6.3.5] we then have that in fact $u \in H^{k+2}\left(\mathfrak{z}^{\circ}\right) \subset$ $C^{k}(\mathfrak{z})$, and furthermore that there is a constant $C_{k}>0$ such that (43) holds. 
Similarly, we have

Lemma 9.2. Fix $g \in C([0, \bar{D}])$ and two constants $\kappa_{1}$ and $\kappa_{2}$ in $\mathbb{R}$. Then there is a unique solution $u_{g, \kappa_{1}, \kappa_{2}} \in C^{2}([0, \bar{D}])$ of the $P D E$

$$
\begin{aligned}
\mathscr{L}_{D} u_{g, \kappa_{1}, \kappa_{2}} & =g \quad \text { in }(0, \bar{D}), \\
u_{g, \kappa_{1}, \kappa_{2}}(0) & =\kappa_{1}, \\
u_{g, \kappa_{1}, \kappa_{2}}(\bar{D}) & =\kappa_{2} .
\end{aligned}
$$

Fix next a nonnegative integer $k \geq 0$. If $g \in C^{k}([0, \bar{D}])$, then we have that $u_{g, \kappa_{1}, \kappa_{2}} \in$ $C^{k+1}([0, \bar{D}])$, and there is a constant $C_{k}>0$ such that

$$
\left\|u_{g, \kappa_{1}, \kappa_{2}}\right\|_{C^{k+1}((0, \bar{D}))} \leq C_{k}\left\{\|g\|_{C^{k}([0, \bar{D}])}+\left|\kappa_{1}\right|+\left|\kappa_{2}\right|\right\}
$$

for all $g \in C^{k}([0, \bar{D}])$ and $\kappa_{1}$ and $\kappa_{2}$ in $\mathbb{R}$.

Proof. We can explicitly compute things here. Define

$$
I(d) \stackrel{\text { def }}{=} 2 \int_{0}^{d} \frac{b_{D}(s)}{\sigma_{D}^{2}(s)} d s
$$

for all $d \in[0, \bar{D}]$ and note that $I \in C^{\infty}([0, \bar{D}])$. Define

$$
\begin{aligned}
u_{g, \kappa_{1}, \kappa_{2}}(d)=\kappa_{1} \frac{\int_{s=d}^{\bar{D}} e^{-I(s)} d s}{\int_{s=0}^{\bar{D}} e^{-I(s)} d s} & +\kappa_{2} \frac{\int_{s=0}^{d} e^{-I(s)} d s}{\int_{s=0}^{\bar{D}} e^{-I(s)} d s} \\
+2 \int_{s=0}^{d} & \int_{r=0}^{s} \frac{g(r)}{\sigma_{D}^{2}(r)} e^{-(I(s)-I(r))} d r d s \\
& -2 \frac{\int_{s=0}^{d} e^{-I(s)} d s}{\int_{s=0}^{\bar{D}} e^{-I(s)} d s} \int_{s=0}^{\bar{D}} \int_{r=0}^{s} \frac{g(r)}{\sigma_{D}^{2}(r)} e^{-(I(s)-I(r))} d r d s
\end{aligned}
$$

for all $d \in[0, \bar{D}]$; all the stated results follow from this.

Actually, if we have $g \in C^{k}([0, \bar{D}])$, then we can bound $\left\|u_{g, \kappa_{1}, \kappa_{2}}\right\|_{C^{k+2}([0, \bar{D}])}$ by $\|g\|_{C^{k}([0, \bar{D}])}$, but in the interest of keeping things similar to Lemma 9.1 , we have stated a weaker result; see also Remark 9.3

Proof of Lemma 4.4. Combine together the results of Lemmas 9.1 and 9.2

Next, let's give the proof of Lemma 4.5.

Proof of Lemma 4.5. Step 1. Since $\lim _{\substack{x \rightarrow \partial \mathfrak{z} \\ x \in \mathfrak{z}^{\circ}}}(\mathscr{L} f)(x)$ exists, $\mathscr{L} f$ is uniformly continuous on $\mathfrak{z}^{\circ}$. Thus, we can find an extension $F \in C_{c}\left(\mathbb{R}^{2}\right)$ of $\mathscr{L} f$. Fix next a mollifier function $\hbar \in C_{c}^{\infty}\left(\mathbb{R}^{2}\right)$ such that $\hbar$ is even and $\int_{z \in \mathbb{R}^{2}} \hbar(z) d z=1$, and define

$$
F^{\varepsilon}(x) \stackrel{\text { def }}{=} \varepsilon^{-2 \nu^{\prime}} \int_{z \in \mathbb{R}^{2}} \hbar\left(\frac{x-z}{\varepsilon^{\nu^{\prime}}}\right) F(z) d z
$$

for all $x \in \mathbb{R}^{2}$. Then $\lim _{\varepsilon \rightarrow 0}\left\|F^{\varepsilon}-\left(\mathscr{L}^{\dagger} f\right)\right\|_{C\left(\mathfrak{z}^{\circ}\right)}=0$ and

$$
\sup _{\varepsilon>0} \varepsilon^{k \nu^{\prime}}\left\|F^{\varepsilon}\right\|_{C^{k}\left(\mathbb{R}^{2}\right)}<\infty
$$

for each integer $k \geq 0$. 
Step 2. Use Lemma 9.1 to solve the PDE

$$
\begin{aligned}
\mathscr{L} f^{\varepsilon} & =F^{\varepsilon} \quad \text { in } \mathfrak{z}^{\circ}, \\
f^{\varepsilon} & =f(\star) \quad \text { on } \partial \mathfrak{z} .
\end{aligned}
$$

Thus $\lim _{\varepsilon \rightarrow 0}\left\|\mathscr{L} f^{\varepsilon}-\mathscr{L} f\right\|_{C\left(\mathfrak{z}^{\circ}\right)}=0$. By Step 1 and the results of Lemma 9.1 we thus know that $\lim _{\varepsilon \rightarrow 0}\left\|f^{\varepsilon}-f\right\|_{H^{2}\left(\mathfrak{z}^{\circ}\right)}=0$ and $\lim _{\varepsilon \rightarrow 0}\left\|f^{\varepsilon}-f\right\|_{C(\mathfrak{z})}=0$. For each integer $k \geq 0$,

$$
\sup _{\varepsilon>0} \varepsilon^{k \nu^{\prime}}\left\|f^{\varepsilon}\right\|_{C^{k}(\mathfrak{z})}<\infty .
$$

Step 3. To finish, we need to extend $f^{\varepsilon}$ (to $\left.Z_{1}\right)$. Suppose that $\tilde{F} \in C_{c}^{7}\left(\mathbb{R} \times \mathbb{R}_{+}\right)$. Let $\zeta \in C_{c}^{\infty}(\mathbb{R})$ be such that $\zeta(\vartheta)=1$ if $|\vartheta|<1$. Define

$$
\hat{F}(x, y) \stackrel{\text { def }}{=} \begin{cases}\tilde{F}(x, y) & \text { if } x \in \mathbb{R}, y \geq 0, \\ \zeta(y) \sum_{j \leq 7} \frac{1}{j !} \frac{\partial^{j} \tilde{F}}{\partial y^{j}}(x, 0) y^{j} & \text { if } x \in \mathbb{R}, y<0 .\end{cases}
$$

Locally using this construction near $\partial \mathfrak{z}$, we get the desired extension.

Similarly, we give the

Proof of Lemma4.6. Step 1. Since the limits

$$
\begin{aligned}
& \lim _{d \searrow 0}\left(\mathscr{L}_{D} f_{D}\right)(d)=\lim _{\substack{[x] \rightarrow \star \\
[x] \in \Gamma}}\left(\mathscr{L}^{\dagger} f\right)([x]), \\
& \lim _{d \nearrow \bar{D}}\left(\mathscr{L}_{D} f_{D}\right)(d)=\lim _{\substack{[x] \rightarrow \circledast \circledast \\
[x] \in \Gamma}}\left(\mathscr{L}^{\dagger} f\right)([x])
\end{aligned}
$$

exist, $\mathscr{L}_{D} f_{D}$ is uniformly continuous on $(0, \bar{D})$. Thus, we can find an extension $F \in C_{c}(\mathbb{R})$ of $\mathscr{L}_{D} f_{D}$. Fix next a mollifier function $\hbar \in C_{c}^{\infty}(\mathbb{R})$ such that $\hbar$ is even and $\int_{z \in \mathbb{R}} \hbar(z) d z=1$, and define

$$
F^{\varepsilon}(d) \stackrel{\text { def }}{=} \varepsilon^{-\nu^{\prime}} \int \hbar\left(\frac{d-z}{\varepsilon^{\nu^{\prime}}}\right) F(z) d z
$$

for all $d \in \mathbb{R}$. Then $\lim _{\varepsilon \rightarrow 0}\left\|F^{\varepsilon}-\left(\mathscr{L}^{\dagger} f\right)_{D}\right\|_{C((0, \bar{D}))}=0$ and

$$
\sup _{\varepsilon>0} \varepsilon^{k \nu^{\prime}}\left\|F^{\varepsilon}\right\|_{C^{k}(\mathbb{R})}<\infty
$$

for each integer $k \geq 0$,

Step 2. Use Lemma 9.2 to solve the PDE

$$
\begin{aligned}
\mathscr{L}_{D} f_{\Gamma}^{\varepsilon} & =F^{\varepsilon} \quad \text { in }(0, \bar{D}), \\
f_{\Gamma}^{\varepsilon}(0) & =f(\star), \\
f_{\Gamma}^{\varepsilon}(\bar{D}) & =f(\circledast) .
\end{aligned}
$$

By Step 1 and the results of Lemma 9.2, we thus know that $\lim _{\varepsilon \rightarrow 0}\left\|f^{\varepsilon}-f\right\|_{C^{1}([0, \bar{D}])}$ $=0$ and that

$$
\sup _{\varepsilon>0} \varepsilon^{k \nu^{\prime}}\left\|f^{\varepsilon}\right\|_{C^{k}([0, \bar{D}])}<\infty
$$

for each integer $k>0$. 
Step 3. To finish, we need to extend $f_{\Gamma}^{\varepsilon}\left(\right.$ to $\left.Z_{2}\right)$. Suppose that $\tilde{F} \in C_{c}^{7}\left(\mathbb{R}_{+}\right)$. Let $\zeta \in C_{c}^{\infty}(\mathbb{R})$ be such that $\zeta(\vartheta)=1$ if $|\vartheta|<1$. Define

$$
\hat{F}(y) \stackrel{\text { def }}{=} \begin{cases}\tilde{F}(y) & \text { if } y \geq 0, \\ \zeta(y) \sum_{j \leq 7} \frac{1}{j !} \frac{d^{j} \tilde{F}}{\partial y^{j}}(0) y^{j} & \text { if } y<0 .\end{cases}
$$

Locally using this construction near 0 and $\bar{D}$, we get the desired extension.

Remark 9.3. The facts that $\left\|\tilde{f}_{\mathfrak{z}}^{\varepsilon}-f\right\|_{H^{2}\left(\mathfrak{z}^{\circ}\right)} \rightarrow 0$ and $\left\|f_{\Gamma}^{\varepsilon}-f_{D}\right\|_{C^{1}([0, \bar{D}])} \rightarrow 0$ are exactly what is needed to approximate $f$ with $\tilde{f}^{\varepsilon}$ in $\mathscr{G}_{\text {inner }}$ and $\mathscr{G}_{\text {outer }}$; see Lemmas 9.8 9.10).

Next, let's make an expansion of test functions near $\partial \mathfrak{z}$. For functions on $[0, \bar{D}]$, this will simply be Taylor's theorem. We also derive a similar expression for functions on $\mathfrak{z}$ which are constant on $\partial \mathfrak{z}$.

Lemma 9.4. First, fix $F \in C^{N}(\mathbb{R})$ for some integer $N \geq 2$. There is a function \urcorner$_{\Gamma, F} \in C^{N-2}(\mathbb{R})$ such that

$$
\left.F(d)=F(0)+d \dot{F}(d)+d^{2}\right\urcorner_{\Gamma, F}(d)
$$

for all $d \in(-\varkappa, \varkappa)$. For each nonnegative integer $k \leq N-2$, there is a constant $C_{k}>0$ such that

$$
\|\rceil_{\Gamma, F}\left\|_{C^{k}((-\varkappa, \varkappa))} \leq C_{k}\right\| F \|_{C^{k+2}((-\varkappa, \varkappa))} .
$$

Next, fix $F \in C^{N}\left(\mathbb{R}^{2}\right)$ for some integer $N \geq 2$ such that $\left.F\right|_{\partial \mathfrak{z}} \equiv \bar{F}$ (where $\bar{F}$ is a constant). Then

$$
\langle d F, d \mathfrak{D}\rangle(x)=\frac{(\nabla F, \nabla \mathfrak{D})_{T \mathbb{R}^{2}}(x)}{\|\nabla \mathfrak{D}(x)\|_{T \mathbb{R}^{2}}^{2}}\langle d \mathfrak{D}, d \mathfrak{D}\rangle(x)
$$

for all $x \in \partial \mathfrak{z}$ and secondly there is a function $\urcorner_{\mathfrak{z}, F} \in C^{N-2}\left(\mathbb{R}^{2}\right)$ such that

$$
\left.F(x)=\bar{F}+\mathfrak{D}(x) \frac{\langle d F, d \mathfrak{D}\rangle}{\langle d \mathfrak{D}, d \mathfrak{D}\rangle}(x)+\mathfrak{D}^{2}(x)\right\rceil_{\mathfrak{z}, F}(x)
$$

for all $x \in \mathcal{N}$. For each nonnegative integer $k \leq N-2$, there is a constant $C_{k}>0$ such that

$$
\|\urcorner_{\mathfrak{z}, F}\left\|_{C^{k}(\mathcal{N})} \leq C_{k}\right\| F \|_{C^{k+2}(\mathcal{N})} .
$$

Proof. When $F \in C^{N}(\mathbb{R})$, we directly have via Taylor's theorem

$$
\urcorner_{\Gamma, F}(d)=\int_{r=0}^{1} r \ddot{F}(r d) d r
$$

for all $d \in \mathbb{R}$. The bounds on $\rceil_{\Gamma, F}$ easily follow from this.

Consider next $F \in \mathbb{R}^{2}$ as required, and fix $x \in \partial \mathfrak{z}$. Since $F$ is constant on $\partial \mathfrak{z}$, $(\nabla F, \bar{\nabla} \mathfrak{D})_{T \mathbb{R}^{2}}(x)=0$. Consequently (by standard projection arguments),

$$
\nabla F(x)=\frac{(\nabla F, \nabla \mathfrak{D})_{T \mathbb{R}^{2}}(x)}{\|\nabla \mathfrak{D}(x)\|_{T \mathbb{R}^{2}}^{2}} \nabla \mathfrak{D}(x) .
$$

Since the map $\iota: T_{x} \mathbb{R}^{2} \rightarrow T_{x}^{*} \mathbb{R}^{2}$ defined by $\iota \nabla \phi(x) \stackrel{\text { def }}{=} d \phi(x)\left(\right.$ for $\left.\phi \in C^{1}\left(\mathbb{R}^{2}\right)\right)$ is linear,

$$
d F(x)=\frac{(\nabla F, \nabla \mathfrak{D})_{T \mathbb{R}^{2}}(x)}{\|\nabla \mathfrak{D}(x)\|_{T \mathbb{R}^{2}}^{2}} d \mathfrak{D}(x) .
$$

This implies (45). 
Fix next a flow $\left\{\underline{\phi}_{t} ; t \in \mathbb{R}\right\}$ of diffeomorphisms of $\mathbb{R}^{2}$ such that

$$
\begin{aligned}
& \dot{\phi}_{t}(x)=-\frac{\nabla \mathfrak{D}}{\|\nabla \mathfrak{D}\|_{T \mathbb{R}^{2}}^{2}}\left(\underline{\phi}_{t}(x)\right), \quad t \in \mathbb{R}, \underline{\phi}_{t}(x) \in \mathcal{N}, \\
& \underline{\phi}_{0}(x)=x .
\end{aligned}
$$

Then $\mathfrak{D}\left(\underline{\phi}_{\mathfrak{D}(x)}(x)\right)=0$ for $x \in \mathcal{N}$. We apply the Taylor remainder theorem to the function $t \mapsto F\left(\underline{\phi}_{t \mathfrak{D}(x)}(x)\right)$ to find that

$$
\left.F(x)=\bar{F}+\mathfrak{D}(x) \frac{(\nabla F, \nabla \mathfrak{D})_{T \mathbb{R}^{2}}}{\|\nabla \mathfrak{D}(x)\|_{T \mathbb{R}^{2}}^{2}}(x)+\mathfrak{D}^{2}(x)\right\urcorner_{\mathfrak{z}, F}^{1}(x)
$$

where

$$
\urcorner_{\mathfrak{z}, F}^{1}(x)=-\int_{s=0}^{1}(1-s)\left(\frac{\nabla \mathfrak{D}}{\|\nabla \mathfrak{D}\|_{T \mathbb{R}^{2}}^{2}}, \nabla\left(\frac{(\nabla F, \nabla \mathfrak{D})_{T \mathbb{R}^{2}}}{\|\nabla \mathfrak{D}\|_{T \mathbb{R}^{2}}^{2}}\right)\right)_{T \mathbb{R}^{2}}\left(\underline{\phi}_{s \mathfrak{D}(x)}(x)\right) d s .
$$

Note now that in light of (45), we have that

$$
\begin{aligned}
\frac{(\nabla F, \nabla \mathfrak{D})_{T \mathbb{R}^{2}}}{\|\nabla \mathfrak{D}(x)\|_{T \mathbb{R}^{2}}^{2}}(x)- & \frac{\langle d F, d \mathfrak{D}\rangle}{\langle d \mathfrak{D}, d \mathfrak{D}\rangle}(x) \\
=\frac{(\nabla F, \nabla \mathfrak{D})_{T \mathbb{R}^{2}}}{\|\nabla \mathfrak{D}(x)\|_{T \mathbb{R}^{2}}^{2}}(x)-\frac{(\nabla F, \nabla \mathfrak{D})_{T \mathbb{R}^{2}}}{\|\nabla \mathfrak{D}(x)\|_{T \mathbb{R}^{2}}^{2}}\left(\underline{\phi}_{\mathfrak{D}(x)}(x)\right) & \\
\quad+\frac{\langle d F, d \mathfrak{D}\rangle}{\langle d \mathfrak{D}, d \mathfrak{D}\rangle}\left(\underline{\phi}_{\mathfrak{D}(x)}(x)\right)- & \frac{\langle d F, d \mathfrak{D}\rangle}{\langle d \mathfrak{D}, d \mathfrak{D}\rangle}(x) \\
& \left.=-\mathfrak{D}(x)\rceil_{\mathfrak{z}, F}^{2}(x)+\mathfrak{D}(x)\right\rceil_{\mathfrak{z}, F}^{3}(x)
\end{aligned}
$$

where

$$
\begin{aligned}
& \neg_{\mathfrak{z}, F}^{2}(x) \stackrel{\text { def }}{=}-\int_{0}^{1}\left(\frac{\nabla \mathfrak{D}}{\|\nabla \mathfrak{D}\|_{T \mathbb{R}^{2}}}, \nabla\left(\frac{(\nabla F, \nabla \mathfrak{D})_{T \mathbb{R}^{2}}}{\|\nabla \mathfrak{D}\|_{T \mathbb{R}^{2}}^{2}}\right)\right)_{T \mathbb{R}^{2}}\left(\underline{\phi}_{s \mathfrak{D}(x)}(x)\right) d s, \\
& \neg_{\mathfrak{z}, F}^{3}(x) \stackrel{\text { def }}{=}-\int_{0}^{1}\left(\frac{\nabla \mathfrak{D}}{\|\nabla \mathfrak{D}\|_{T \mathbb{R}^{2}}}, \nabla\left(\frac{\langle d F, d \mathfrak{D}\rangle}{\langle d \mathfrak{D}, d \mathfrak{D}\rangle}\right)\right)_{T \mathbb{R}^{2}}\left(\underline{\phi}_{s \mathfrak{D}(x)}(x)\right) d s .
\end{aligned}
$$

Set $\left.\left.\left.\urcorner_{\mathfrak{z}, F} \stackrel{\text { def }}{=}\right\urcorner_{\mathfrak{z}, F}^{1}-\right\urcorner_{\mathfrak{z}, F}^{2}+\right\rceil_{\mathfrak{z}, F}^{3}$. The bounds on $\urcorner_{\mathfrak{z}, F}$ follow from the explicit formulae.

Our next task is to show that $\mathscr{G}_{\text {inner }} f$ and $\mathscr{G}_{\text {outer }} f$ are well-defined for $f \in \mathscr{D}^{\dagger}$. Let's do the easy part first.

Lemma 9.5. The quantity $\mathscr{G}_{\text {outer }} f$ is well-defined and $\mathscr{G}_{\text {outer }} f=\sigma_{D}^{2}(0) \dot{f}_{D}(0)$.

Proof. For $x \in \overline{\mathbf{S}} \backslash \mathfrak{z}$,

$$
\langle d f, d \tilde{\mathfrak{D}}\rangle_{\text {ave }}([x])=\dot{f}_{D}(\mathfrak{D}(x))\langle d \tilde{\mathfrak{D}}, d \tilde{\mathfrak{D}}\rangle_{\text {ave }}([x])=\dot{f}_{D}(\mathfrak{D}(x)) \sigma_{D}^{2}(\mathfrak{D}(x)) .
$$

By Lemma $4.4 f_{D}(0)=\lim _{d \searrow 0} \dot{f}_{D}(d)$ exists.

We next want to study $\mathscr{G}_{\text {inner }} f$. Again, we arm ourselves with a tool from the theory of PDE's. For each $d \in(-\varkappa, \varkappa)$, we let $\mathbf{T}_{d}: H^{1}\left(\mathfrak{z}^{\circ}\right) \rightarrow L^{2}\left(\mathfrak{D}^{-1}(d)\right)$ be the standard trace operator [6, Section 5.5]. For $\varphi \in H^{1}\left(\mathfrak{z}^{\circ}\right)$, define

$$
\left(\mathbf{A}^{T} \varphi\right)(d) \stackrel{\text { def }}{=} \frac{\int_{y \in \mathfrak{D}^{-1}(d)}\left(\mathbf{T}_{d} \varphi\right)(y)\|\bar{\nabla} \mathfrak{D}(y)\|_{T \mathbb{R}^{2}}^{-1} \mathscr{H}^{1}(d y)}{\int_{y \in \mathfrak{D}^{-1}(d)}\|\bar{\nabla} \mathfrak{D}(y)\|_{T \mathbb{R}^{2}}^{-1} \mathscr{H}^{1}(d y)}
$$


for all $d \in(-\varkappa, 0]$. We note that if in addition $\varphi \in C(\mathfrak{z})$, then $\mathbf{A}^{T} \varphi=\mathbf{A}^{D} \varphi$ on $(-\varkappa, 0]$, where $\mathbf{A}^{D}$ is as in (16) (i.e., $\mathbf{A}^{T}$ is an extension of $\mathbf{A}^{D}$ to $\left.H^{1}\left(\mathfrak{z}^{\circ}\right)\right)$. We will also use the following auxiliary result on the smoothness of $\mathbf{A}^{D}$.

Lemma 9.6. There is a constant $C>0$ such that

$$
\left|\left(\mathbf{A}^{D} \varphi\right)(d)-\left(\mathbf{A}^{T} \varphi\right)\left(d^{\prime}\right)\right| \leq C\|\varphi\|_{H^{1}\left(\mathbb{R}^{2}\right)} \sqrt{\left|d-d^{\prime}\right|}
$$

for all $d$ and $d^{\prime}$ in $(-\varkappa, \varkappa)$ and all $\varphi \in H^{2}\left(\mathbb{R}^{2}\right)$.

Proof. Fix $\varphi \in C^{\infty}\left(\mathbb{R}^{2}\right)$ whose support is contained in $(-1,1)^{2}$. Then for any $-1<y_{1}<y_{2}<1$,

$$
\begin{array}{r}
\left|\int_{x=-1}^{1} \varphi\left(x, y_{2}\right) d x-\int_{x=-1}^{1} \varphi\left(x, y_{1}\right) d x\right|=\left|\int_{x=-1}^{1} \int_{y=y_{1}}^{y_{2}} \frac{\partial \varphi}{\partial y}(x, y) d y d x\right| \\
\leq \sqrt{\left|y_{2}-y_{1}\right|} \sqrt{\int_{x=-1}^{1} \int_{y=y_{1}}^{y_{2}}\left(\frac{\partial \varphi}{\partial y}(x, y)\right)^{2} d y d x}
\end{array}
$$

By standard transformations, approximations, and covering arguments, we get the stated result.

We now prove

Lemma 9.7. The quantity $\mathscr{G}_{\text {inner }} f$ is well-defined and

$$
\mathscr{G}_{\text {inner }} f=\left(\mathbf{A}^{T}\langle d f, d \mathfrak{D}\rangle\right)(0)=\left(\mathbf{A}^{T} \frac{(\nabla f, \nabla \mathfrak{D})_{T \mathbb{R}^{2}}\langle d \mathfrak{D}, d \mathfrak{D}\rangle}{\|\bar{\nabla} \mathfrak{D}\|_{T \mathbb{R}^{2}}^{2}}\right)(0),
$$

both of these quantities begin well-defined.

Proof. First, note that since $f \in H^{2}\left(\mathfrak{z}^{\circ}\right)$, the arguments of $\mathbf{A}^{T}$ are both in $H^{1}\left(\mathfrak{z}^{\circ}\right)$, so both expressions for $\mathscr{G}_{\text {inner }} f$ are indeed well-defined.

Next, we observe that

$$
\mathscr{G}_{\text {inner }} f=\lim _{d \nearrow 0}\left(\mathbf{A}^{T}\langle d f, d \mathfrak{D}\rangle\right)(d),
$$

if this limit exists. We approximate using the $\tilde{f}_{\mathfrak{z}}^{\varepsilon}$ 's of Lemma 4.5. By standard properties of the trace operator, we have that there is a $C>0$ such that

$$
\left|\left(\mathbf{A}^{T}\langle d f, d \mathfrak{D}\rangle\right)(d)-\left(\mathbf{A}^{T}\left\langle d \tilde{f}_{\mathfrak{z}}^{\varepsilon}, d \mathfrak{D}\right\rangle\right)(d)\right| \leq C\left\|f-\tilde{f}_{\mathfrak{z}}^{\varepsilon}\right\|_{H^{2}\left(\mathfrak{z}^{\circ}\right)}
$$

for all $-\varkappa<d \leq 0$. Using Lemma 9.6 and the fact that $\mathbf{A}^{T}$ is an extension of $\mathbf{A}^{D}$, we get that for some $C>0$,

$$
\left|\left(\mathbf{A}^{T}\langle d f, d \mathfrak{D}\rangle\right)(d)-\left(\mathbf{A}^{T}\langle d f, d \mathfrak{D}\rangle\right)(0)\right| \leq 2 C\left\|f-\tilde{f}_{\mathfrak{z}}^{\varepsilon}\right\|_{H^{2}\left(\mathfrak{z}^{\circ}\right)}+C\left\|\tilde{f}_{\mathfrak{z}}^{\varepsilon}\right\|_{H^{2}\left(\mathfrak{z}^{\circ}\right)} \sqrt{|d|} .
$$

First let $\varepsilon$ tend to zero and then let $d$ tend to zero. This gives us the existence of $\mathscr{G}_{\text {inner }} f$ and its first representation. To get the second representation, note that by Lemma 9.4

$$
\left(\mathbf{A}^{T}\left\langle d \tilde{f}_{\mathfrak{z}}^{\varepsilon}, d \mathfrak{D}\right\rangle\right)(0)=\left(\mathbf{A}^{T} \frac{\left(\nabla \tilde{f}_{\mathfrak{z}}^{\varepsilon}, \nabla \mathfrak{D}\right)_{T \mathbb{R}^{2}}\langle d \mathfrak{D}, d \mathfrak{D}\rangle}{\|\bar{\nabla} \mathfrak{D}\|_{T \mathbb{R}^{2}}^{2}}\right)
$$

for every $\varepsilon>0$. By the properties of the trace operator and since

$$
\lim _{\varepsilon \rightarrow 0}\left\|f-\tilde{f}_{\mathfrak{z}}^{\varepsilon}\right\|_{H^{2}\left(\mathfrak{z}^{\circ}\right)}=0
$$

we get the alternate representation for $\mathscr{G}_{\text {inner }} f$. 
The next task of this section is to bound some of the appropriate terms in the proofs of Lemmas 7.1 and 7.2 (e.g., the $\phi_{i}^{\varepsilon}$ 's of (34) and the $\omega_{i}(\varepsilon)$ 's of (36)).

Lemma 9.8. We have that $\lim _{\varepsilon \rightarrow 0} \omega_{1}(\varepsilon)=0$ and $\sup _{\varepsilon>0} \varepsilon^{5 \nu^{\prime}}\left\|\phi_{1}^{\varepsilon}\right\|_{C^{4}(\mathcal{N})}<\infty$.

Proof. It is clear that all derivatives of $\phi_{1}^{\varepsilon}$ of order 4 or less involve derivatives of $\tilde{f}_{\mathfrak{z}}^{\varepsilon}$ and $f_{\Gamma}^{\varepsilon}$ of order 5 or less; by the results of Lemmas 4.5 and 4.6 we thus get the stated bound on $\left\|\phi_{1}^{\varepsilon}\right\|_{C^{4}(\mathcal{N})}$.

It is easy to see that

$$
\begin{aligned}
\left|\left(\mathbf{A}^{D} \phi_{1}^{\varepsilon}\right)(x)\right| \leq & \left|\left(\mathbf{A}^{D}\left\langle d \tilde{f}_{\mathfrak{z}}^{\varepsilon}, d \mathfrak{D}\right\rangle\right)(\mathfrak{D}(x))-\left(\mathbf{A}^{D}\left\langle d \tilde{f}_{\Gamma}^{\varepsilon}, d \mathfrak{D}\right\rangle\right)(\mathfrak{D}(x))\right| \\
\leq & \left|\left(\mathbf{A}^{D}\left\langle d \tilde{f}_{\mathfrak{z}}^{\varepsilon}, d \mathfrak{D}\right\rangle\right)(\mathfrak{D}(x))-\left(\mathbf{A}^{D}\left\langle d \tilde{f}_{\mathfrak{z}}^{\varepsilon}, d \mathfrak{D}\right\rangle\right)(0)\right| \\
\quad+\left|\left(\mathbf{A}^{T}\left\langle d \tilde{f}_{\mathfrak{z}}^{\varepsilon}, d \mathfrak{D}\right\rangle\right)(0)-\mathscr{G}_{\text {inner }} f\right| & \\
& +\left|\mathscr{G}_{\text {outer }} f-\dot{f}_{\Gamma}^{\varepsilon}(0) \sigma_{D}^{2}(0)\right|+\left|\dot{f}_{\Gamma}^{\varepsilon}(0) \sigma_{D}^{2}(0)-\dot{f}_{\Gamma}^{\varepsilon}(\mathfrak{D}(x)) \sigma_{D}^{2}(\mathfrak{D}(x))\right|
\end{aligned}
$$

for any $x \in \mathcal{N}$. We now collect together a number of results we have proved. We use the properties of the trace operators, Lemmas 9.5 9.6, and 9.7, and the approximation results of Lemmas 4.5 and 4.6. We find that there is a constant $C>0$ such that

$$
\left|\omega_{i}(\varepsilon)\right| \leq C\left\{\varepsilon^{-2 \nu^{\prime}+\nu / 2}+\left\|\tilde{f}_{\mathfrak{z}}^{\varepsilon}-f\right\|_{H^{2}\left(\mathfrak{z}^{\circ}\right)}+\left\|f_{D}-f_{\Gamma}^{\varepsilon}\right\|_{C^{1}([0, \bar{D}])}+\varepsilon^{-2 \nu^{\prime}+\nu}\right\}
$$

for all $\varepsilon^{\nu}<\mathfrak{D}(x)<2 \varepsilon^{\nu}$. By our choice of $\nu$ and $\nu^{\prime}$, we have the stated result 8 .

Secondly, we have

Lemma 9.9. We have that $\lim _{\varepsilon \rightarrow 0} \omega_{2}(\varepsilon)=0$ and $\sup _{\varepsilon>0} \varepsilon^{6 \nu^{\prime}}\left\|\phi_{1}^{\varepsilon}\right\|_{C^{4}(\mathcal{N})}<\infty$.

Proof. Recall that $\left.\tilde{f}_{\mathfrak{z}}^{\varepsilon}\right|_{\partial \mathfrak{z}}=f_{\Gamma}^{\varepsilon}(0)=f(\star)$. We can then use Lemma 9.4 to write that

$$
\begin{aligned}
\frac{\tilde{f}_{\mathfrak{z}}^{\varepsilon}(x)-\tilde{f}_{\Gamma}^{\varepsilon}(x)}{\mathfrak{D}(x)}= & \frac{\tilde{f}_{\mathfrak{z}}^{\varepsilon}(x)-f(\star)}{\mathfrak{D}(x)}-\frac{f_{\Gamma}^{\varepsilon}(\mathfrak{D}(x))-f(\star)}{\mathfrak{D}(x)} \\
& \left.\left.=\frac{\left\langle d \tilde{f}_{\mathfrak{z}}^{\varepsilon}, d \mathfrak{D}\right\rangle(x)}{\langle d \mathfrak{D}, d \mathfrak{D}\rangle(x)}+\mathfrak{D}(x)\right\rceil_{\mathfrak{z}, \tilde{f}_{\mathfrak{z}}^{\varepsilon}}-\dot{f}_{\Gamma}^{\varepsilon}(\mathfrak{D}(x))-\mathfrak{D}(x)\right\rceil_{\Gamma, f_{\Gamma}^{\varepsilon}}(\mathfrak{D}(x))
\end{aligned}
$$

for all $x \in \mathcal{N}$. Using the bounds of Lemma 9.4 we see that we can bound all derivatives of $\phi_{2}^{\varepsilon}$ of order 4 or less by $\left\|\tilde{f}_{\mathfrak{z}}^{\varepsilon}\right\|_{C^{6}(\mathcal{N})}$ and $\left\|f_{\Gamma}^{\varepsilon}\right\|_{C^{6}((-\varkappa, \varkappa))}$; by the bounds of Lemmas 4.5 and 4.6, we get the stated bound on $\left\|\phi_{2}^{\varepsilon}\right\|_{C^{4}(\mathcal{N})}$. Similarly, we see that there is a constant $C>0$ such that

$$
\left|\phi_{2}^{\varepsilon}(x)-\phi_{1}^{\varepsilon}(x)\right| \leq C \varepsilon^{\nu-2 \nu^{\prime}}
$$

for all $x \in \mathbf{S}$ such that $\varepsilon^{\nu}<\mathfrak{D}(x)<2 \varepsilon^{\nu}$. By our choice of $\nu$ and $\nu^{\prime}$, we have that $\lim _{\varepsilon \rightarrow 0} \omega_{2}(\varepsilon)=0$ since $\lim _{\varepsilon \rightarrow 0} \omega_{1}(\varepsilon)=0$ (i.e., Lemma 9.8) 9 .

${ }^{8}$ We use here that

$$
\nu^{\prime}<\min \left\{\frac{\nu}{4}, \frac{\nu}{2}\right\}=\frac{\nu}{4}=\frac{1}{2(n+1)}
$$

${ }^{9}$ We use here that

$$
\nu^{\prime}<\frac{\nu}{2}=\frac{1}{n+1} .
$$


Thirdly, we have

Lemma 9.10. There is a $\phi_{3}^{\varepsilon} \in C^{4}\left(\mathbb{R}^{2}\right)$ such that (38) holds. We may assume that $\mathbf{A}^{D} \phi_{3}^{\varepsilon} \equiv 0$ and that $\sup _{\varepsilon>0} \varepsilon^{7 \nu^{\prime}}\left\|\phi_{3}^{\varepsilon}\right\|_{C^{4}(\mathcal{N})}<\infty$.

Proof. By Lemma 9.4 we may take

$$
\left.\phi_{3}^{\varepsilon}(x)=\left(\bar{\nabla} \mathfrak{D}, \nabla \frac{\left\langle d \tilde{f}_{\mathfrak{z}}^{\varepsilon}, d \mathfrak{D}\right\rangle}{\langle d \mathfrak{D}, d \mathfrak{D}\rangle}\right)_{T \mathbb{R}^{2}}(x)+\mathfrak{D}(x)\left(\bar{\nabla} \mathfrak{D}_{T \mathbb{R}^{2}}, \nabla\right\rceil_{\mathfrak{z}, \tilde{f}_{\mathfrak{z}}^{\varepsilon}}^{\varepsilon}\right)(x) .
$$

Thus, derivatives of $\phi_{3}^{\varepsilon}$ of order 4 or less can be bounded by derivatives of $\tilde{f}_{\mathfrak{z}}^{\varepsilon}$ of order 7 or less. The fact that $\mathbf{A}^{D} \phi_{3}^{\varepsilon} \equiv 0$ follows from the fact that $\phi_{3}^{\varepsilon}$ is in the range of $\bar{\nabla} \mathfrak{D}$.

Finally, we prove a result needed in the proof of uniqueness (viz. in Lemma 8.3).

Lemma 9.11. If $f \in \mathscr{D}^{\dagger}$ and $f$ attains its maximum on $\star$, then $\mathscr{G}_{\text {inner }} f \geq 0$.

Proof. Define

$$
u(t) \stackrel{\text { def }}{=} \int_{x \in \partial \mathfrak{z}}\left\{f\left(\underline{\phi}_{t}(x)\right)-f(\star)\right\}\langle d \mathfrak{D}, d \mathfrak{D}\rangle(x)\|\bar{\nabla} \mathfrak{D}(x)\|_{T \mathbb{R}^{2}}^{-1} \mathscr{H}^{1}(d x)
$$

for $0<t<\varkappa$. It is clear (by a calculation similar to the proof of Lemma 9.6) that $u$ is differentiable on $(0, \varkappa)$ and

$$
\lim _{t \searrow 0} \dot{u}(t)=-\mathfrak{T}^{D}(0) \mathscr{G}_{\text {inner }} f
$$

(where $\mathfrak{T}^{D}$ is as in (15)). Furthermore, we have that $\lim _{t \searrow_{0}} u(t)=0$ and that

$$
\int_{t=0}^{\varkappa}|\dot{u}(t)| d t<\infty
$$

(one can bound this in terms of $\|f\|_{H^{2}\left(\mathfrak{z}^{\circ}\right)}$ ). Thus

$$
u(t)=\int_{s=0}^{t} \dot{u}(s) d s
$$

for $0<t<\varkappa$. If $\mathscr{G}_{\text {inner }} f<0$, then $u(t)>0$ for $t>0$ sufficiently small, implying that $f\left(\phi_{t}(x)\right)>f(\star)$ for some $x \in \partial \mathfrak{z}$, contradicting the hypothesis that $f$ attains its maximum at $\star$.

\section{Khasminskit PDE}

Here we prove Proposition 6.5. We will construct $\tilde{\Psi}$ by a Fourier expansion. For each $k \in \mathbb{Z}$, define

$$
\phi_{k} \stackrel{\text { def }}{=} \frac{1}{\alpha(0)} \int_{\theta=0}^{\alpha(0)} \frac{\partial \tilde{\Phi}}{\partial \theta}(\theta, 0) e^{-2 \pi k \theta \imath / \alpha(0)} d \theta
$$

where, as usual, $\imath \stackrel{\text { def }}{=} \sqrt{-1}$. We note that $\phi_{0}=0$ since $\tilde{\Phi}(\alpha(0), 0)=\tilde{\Phi}(0,0)$. Formally, we want to write that

$$
\tilde{\Psi}(\theta, d)=\frac{8}{(n+1)^{2}} \sum_{k \in \mathbb{Z}} \phi_{k} e^{2 \pi k \theta \imath / \alpha(0)} \xi_{k}(d)
$$

The combination of this and footnotes 12 and 648 give the restriction on $\nu^{\prime}$ given in Definition 4.2 . 
for all $\theta \in \mathbb{R}$ and $d>0$ (the term $8 /(n+1)$ provides a convenient normalization). We thus want that $\xi_{k}$ satisfy the PDE

$$
\frac{(n+1)^{2}}{8} \ddot{\xi}_{k}(d)+\frac{n^{2}-1}{8 d} \dot{\xi}_{k}(d)+\frac{2 \pi k n \imath}{\alpha(0)} \xi_{k}(d)=\frac{(n+1)^{2}}{8} \tilde{\varpi}(d)
$$

or equivalently (after some simple algebra) that

$$
\ddot{\xi}_{k}(d)+\frac{1-\nu}{d} \dot{\xi}_{k}(d)+\lambda_{k}^{2} \xi_{k}(d)=\tilde{\varpi}(d)
$$

where

$$
\lambda_{k}^{2}=\frac{2 \pi k n \imath / \alpha(0)}{(n+1)^{2} / 8}=\frac{16 k n \pi \imath}{(n+1)^{2} \alpha(0)} .
$$

Let's choose the $\lambda_{k}$ 's such that their real parts are positive. For positive $k \in \mathbb{Z}$, define

$$
\lambda_{k} \stackrel{\text { def }}{=}|k|^{1 / 2} \frac{4}{n+1} \sqrt{\frac{\pi n}{\alpha(0)}} \frac{1+\imath}{\sqrt{2}}=|k|^{1 / 2} \frac{4}{n+1} \sqrt{\frac{\pi n}{\alpha(0)}} e^{\pi \imath / 4}
$$

and for negative $k \in \mathbb{Z}$, define

$$
\lambda_{k} \stackrel{\text { def }}{=}|k|^{1 / 2} \frac{4}{n+1} \sqrt{\frac{\pi n}{\alpha(0)}} \frac{1-\imath}{\sqrt{2}}=|k|^{1 / 2} \frac{4}{n+1} \sqrt{\frac{\pi n}{\alpha(0)}} e^{-\pi \imath / 4} .
$$

We will show in Lemma 10.2 that (48) has a solution such that

$$
\lim _{d \rightarrow \infty} \xi_{k}(d)=0 \quad \text { and } \quad \lim _{d \searrow 0} \dot{\xi}_{k}(d)=0
$$

(i.e., these are the two boundary conditions which uniquely specify the solution). We will also show in Lemma 10.3 that this solution has other desired properties which we will need.

The $d^{-1}$ term in the first-order part indicates that solutions of Bessel equations are somehow involved. Let's first connect the homogeneous form of (48) to Bessel's ODE and establish a variation of parameters formula to solve the inhomogeneous ODE (48).

Lemma 10.1. Suppose that $J$ is analytic on $\mathbb{C} \backslash \mathbb{R}_{-}$and satisfies Bessel's ODE

$$
\ddot{J}(z)+\frac{\dot{J}(z)}{z}+\left(1-\frac{(\nu / 2)^{2}}{z^{2}}\right) J(z)=0
$$

on $\mathbb{C} \backslash \mathbb{R}_{-}$. Set $\zeta(d) \stackrel{\text { def }}{=}\left(\lambda_{k} d\right)^{\nu / 2} J\left(\lambda_{k} d\right)$ for all $d>0$ (using the principal branch of $\left.z \mapsto z^{\nu / 2}\right)$; then $\zeta:(0, \infty) \rightarrow \mathbb{C}$ is $C^{\infty}$ on $(0, \infty)$ and satisfies the homogeneous $O D E$

$$
\ddot{\zeta}(d)+\frac{1-\nu}{d} \dot{\zeta}(d)+\lambda_{k}^{2} \zeta(d)=0
$$

for all $d>0$. Next, assume that $\zeta$ has no zeroes on $(0, \infty)$ and define

$$
\xi(d) \stackrel{\text { def }}{=} \zeta(d) \int^{d} \frac{1}{u^{1-\nu} \zeta^{2}(u)} \int^{u} \tilde{\varpi}(s) s^{1-\nu} \zeta(s) d s d u
$$

for all $d>0$. If the constants of integration in this formula are chosen so that $\xi$ is well-defined, then $\xi$ is $C^{2}$ on $(0, \infty)$ and satisfies the ODE (48).

Proof. Straightforward. 
We want to use this result to solve (48) with the desired boundary conditions; i.e., we need to select a solution of Bessel's ODE to find a particular solution of (48) and then select constants of integration for the indefinite integrals in (49). We refer to [17] for relevant results about solutions of Bessel's ODE.

For each $v \in \mathbb{R}$, let $J_{v}$ be the Bessel function of order $v$; i.e.,

$$
J_{v}(z)=(z / 2)^{v} \sum_{m=0}^{\infty} \frac{\left(-z^{2} / 4\right)^{m}}{m ! \Gamma(v+m+1)}
$$

for all $z \in \mathbb{C} \backslash \mathbb{R}_{-}$, where we take the principal branch of $(z / 2)^{v}$ and where $\Gamma$ is the standard Gamma function. For $k \in \mathbb{Z} \backslash\{0\}$ and $d>0$, define

$$
\zeta_{k}(z) \stackrel{\text { def }}{=}\left(\lambda_{k} d\right)^{\nu / 2} J_{-\nu / 2}\left(\lambda_{k} d\right)
$$

and then

$$
\xi_{k}(d) \stackrel{\text { def }}{=}-\zeta_{k}(d) \int_{u=d}^{\infty} \frac{1}{u^{1-\nu} \zeta_{k}^{2}(u)} \int_{s=0}^{u} \tilde{\varpi}(s) s^{1-\nu} \zeta_{k}(s) d s d u .
$$

We will prove below that this gives us the the desired solution of (48).

Let's proceed by recording some known properties of Bessel functions which, in addition to the representation (50), we will use in our efforts. We use [1] and [17] as references. First, all of the zeroes of the $J_{v}$ 's are real when $v \geq-1[17 \S 7.6]$ (and note here that $-\nu / 2=-1 /(n+1)>-1)$. Secondly, we have that

$$
J_{v}\left(x e^{ \pm \pi \imath / 2}\right)=\sqrt{\frac{2}{\pi x e^{ \pm \pi \imath / 2}}}\left\{\cos \left(x e^{ \pm \pi \imath / 2}-\frac{v \pi}{2}-\frac{\pi}{4}\right)+e^{x / \sqrt{2}} \mathcal{E}_{v}\left(x e^{ \pm \pi \imath / 2}\right)\right\}
$$

where $\mathcal{E}_{v}$ is some function such that $\lim _{x / \infty}\left|\mathcal{E}_{v}\left(x e^{ \pm \pi \imath / 2}\right)\right| x<\infty$ (see [17 Eq. 4.9.09] and [1, Eq. 9.2.1]). Finally, the derivatives of $J_{v}$ can be understood via the relation

$$
\dot{J}_{v}(z)=\frac{1}{2}\left\{J_{v-1}(z)-J_{v+1}(z)\right\},
$$

which holds for all $z \in \mathbb{C} \backslash \mathbb{R}_{-}$and $v \in \mathbb{R}$ [17] Eq. 2.9.15]. We use all of this to get some relevant bounds on $\zeta_{k}$ 's.

Lemma 10.2. We have that

$$
\lim _{d \searrow 0} \zeta_{k}(d)=\frac{2^{\nu / 2}}{\Gamma(1-\nu / 2)} \quad \text { and } \quad \lim _{d \searrow 0} \dot{\zeta}_{k}(d)=0 .
$$

Furthermore, there is a constant $C>0$ such that

$$
\begin{gathered}
\frac{1}{C} \frac{e^{\left|\lambda_{k}\right| d / \sqrt{2}}}{\left(1+\left|\lambda_{k}\right| d\right)^{(1-\nu) / 2}} \leq\left|\zeta_{k}(d)\right| \leq C \frac{e^{\left|\lambda_{k}\right| d / \sqrt{2}}}{\left(1+\left|\lambda_{k}\right| d\right)^{(1-\nu) / 2}} \\
\left|\dot{\zeta}_{k}(d)\right| \leq C \frac{\left|\lambda_{k}\right|^{2} d e^{\left|\lambda_{k}\right| d / \sqrt{2}}}{\left(1+\left|\lambda_{k}\right| d\right)^{(3-\nu) / 2}}
\end{gathered}
$$

for all $d>0$.

Proof. For convenience define

$$
\zeta^{\circ}(z) \stackrel{\text { def }}{=} z^{\nu / 2} J_{-\nu / 2}(z)=\sum_{m=0}^{\infty} \frac{2^{\nu / 2}\left(-z^{2} / 4\right)^{m}}{m ! \Gamma(-\nu / 2+m+1)}
$$

for all $z \in \mathbb{C} \backslash \mathbb{R}$. Then

$$
\zeta_{k}(d)=\zeta^{\circ}\left(\lambda_{k} d\right)
$$


for all $d>0$. It is easy to see that

$$
\lim _{z \rightarrow 0} \zeta^{\circ}(z)=\frac{2^{\nu / 2}}{\Gamma(-\nu / 2+1)} \quad \text { and } \quad \lim _{z \rightarrow 0} \frac{\dot{\zeta}^{\circ}(z)}{z}=-\frac{2^{\nu / 2}}{2 \Gamma(-\nu / 2+2)} .
$$

The stated results about $\zeta_{k}$ at zero consequently hold. Next, note that

$$
\cos x=\frac{1}{2} e^{-\imath x}\left\{1+e^{2 \imath x}\right\}+\frac{1}{2} e^{\imath x}\left\{1+e^{-2 \imath x}\right\}
$$

for all $x \in \mathbb{C}$ and that for any $v \in \mathbb{R}$,

$$
\begin{aligned}
\imath\left(x e^{\pi / 4}-\frac{v \pi}{2}-\frac{\pi}{4}\right) & =-\frac{x}{\sqrt{2}}+\imath\left\{\frac{x}{\sqrt{2}}-\frac{2 v+1}{4} \pi\right\}, \\
\imath\left(x e^{-\pi \imath / 4}-\frac{v \pi}{2}-\frac{\pi}{4}\right) & =\frac{x}{\sqrt{2}}+\imath\left\{\frac{x}{\sqrt{2}}-\frac{2 v+1}{4} \pi\right\}
\end{aligned}
$$

for all $x>0$. It is thus not hard to see from (52) that for any fixed $v \in \mathbb{R}$, there is a constant $C>0$ such that

$$
\frac{1}{C} \frac{e^{x / \sqrt{2}}}{\sqrt{x}} \leq\left|J_{v}\left(x e^{ \pm \pi \imath / 4}\right)\right| \leq C \frac{e^{x / \sqrt{2}}}{\sqrt{x}}
$$

for all sufficiently large positive real $x$. Using this and taking into account (55) and the fact that all the zeroes of $J_{-\nu / 2}$ are real, we thus have that there is a constant $C>0$ such that

$$
\frac{1}{C} \frac{e^{x / \sqrt{2}}}{(1+x)^{(1-\nu) / 2}} \leq\left|\zeta^{\circ}\left(x e^{ \pm \pi \imath / 4}\right)\right| \leq C \frac{e^{x / \sqrt{2}}}{(1+x)^{(1-\nu) / 2}}
$$

for all $x>0$. This gives us the first bound of (54).

Let's now look at the derivative of $\zeta^{\circ}$. Using (53), we have that

$$
\dot{\zeta}^{\circ}(z)=\frac{\nu}{2} z^{\nu / 2-1} J_{-\nu / 2}(z)+\frac{1}{2} z^{\nu / 2} J_{-\nu / 2-1}(z)-\frac{1}{2} z^{\nu / 2} J_{-\nu / 2+1}(z)
$$

for all $z \in \mathbb{C} \backslash \mathbb{R}_{-}$. Thus by (56), we get that there is a constant $C>0$ such that

$$
\left|\dot{\zeta}^{\circ}\left(x e^{ \pm \pi \imath / 4}\right)\right| \leq C \frac{e^{x / \sqrt{2}}}{x^{(1-\nu) / 2}}
$$

for all $x \geq 1$. Taking into account (55), we have that there is a constant $C>0$ such that

$$
\left|\dot{\zeta}^{\circ}\left(x e^{ \pm \pi \imath / 4}\right)\right| \leq C \frac{x e^{x / \sqrt{2}}}{(1+x)^{(3-\nu) / 2}}
$$

for all $x>0$. This gives us the second bound of (54).

Let's next prove some desired results about $\xi_{k}$.

Lemma 10.3. The right-hand side of equality (51) is well-defined. We have that $\lim _{d \searrow 0} \xi_{k}(d)$ exists and that $\lim _{d \searrow 0} \dot{\xi}_{k}(d)=0$. Furthermore, there is a constant $C>0$ such that

$$
\left|\xi_{k}^{(i)}(d)\right| \leq C\left|\lambda_{k}\right|^{i-(\nu+1) / 2} e^{-d / C}
$$

for all $d>0, k \in \mathbb{Z} \backslash\{0\}$, and $i \in\{0,1\}$ (where $\zeta_{k}^{(i)}$ denotes the $i$-th derivative of $\left.\zeta_{k}\right)$. 
Proof. As we pointed out above, the zeroes of Bessel functions all lie on the real axis; thus the $\zeta_{k}$ 's have no zeroes on $(0, \infty)$. Fix for a moment $k \in \mathbb{Z} \backslash\{0\}$. Note also that since $\tilde{\varpi}$ has compact support, the inner integral is bounded; in light of the exponential growth of $\zeta_{k}$ of Lemma 10.2 we can conclude that $\xi_{k}$ is welldefined on $(0, \infty)$. Since $\lim _{d \searrow 0} \zeta_{k}(d)$ exists but is nonzero, we also can conclude that $\lim _{d \searrow 0} \xi_{k}(d)$ exists (we may use the fact that $\int_{0^{+}} 1 / u^{1-\nu} d u<\infty$ ). We can furthermore note that

$$
\dot{\xi}_{k}(d)=\frac{\dot{\zeta}_{k}(d)}{\zeta_{k}(d)} \xi_{k}(d)+A_{k}(d)
$$

where

$$
A_{k}(d) \stackrel{\text { def }}{=} \frac{1}{d^{1-\nu} \zeta_{k}(d)} \int_{s=0}^{d} \tilde{\varpi}(s) s^{1-\nu} \zeta_{k}(s) d s,
$$

which holds for all $k \in \mathbb{Z} \backslash\{0\}$ and all $d>0$. It is fairly easy to see from this that $\lim _{d \searrow 0} \dot{\xi}_{k}(d)=0$.

For convenience, let's define

$$
\beta_{k} \stackrel{\text { def }}{=}\left|\lambda_{k}\right| / \sqrt{2}
$$

for all $k \in \mathbb{Z} \backslash\{0\}$. Also, let $d^{*}>0$ be such that $\tilde{\varpi}(u)=0$ if $u \geq d^{*}$.

It is easy to see that there is a constant $C>0$ such that

$$
\begin{aligned}
\left|\xi_{k}(d)\right| \leq & C \int_{u=d}^{\infty} \int_{s=0}^{u} \chi_{\left[0, d^{*}\right]}(s) \frac{e^{\beta_{k} d}}{\left(1+\left|\lambda_{k}\right| d\right)^{(1-\nu) / 2}} \frac{\left(1+\left|\lambda_{k}\right| u\right)^{(1-\nu)}}{u^{1-\nu} e^{2 \beta_{k} u}} \\
& \times \frac{s^{1-\nu} e^{\beta_{k} s}}{\left(1+\left|\lambda_{k}\right| s\right)^{(1-\nu) / 2}} d s d u \\
= & C \int_{u=d}^{\infty} \int_{s=0}^{u \wedge d^{*}} e^{\beta_{k} d-2 \beta_{k} u+\beta_{k} s} \\
& \times \frac{\left(1+\left|\lambda_{k}\right| u\right)^{(1-\nu)} s^{1-\nu}}{\left(1+\left|\lambda_{k}\right| d\right)^{(1-\nu) / 2}\left(1+\left|\lambda_{k}\right| s\right)^{(1-\nu) / 2} u^{1-\nu}} d u d u
\end{aligned}
$$

for all $d>0$ and $k \in \mathbb{Z} \backslash\{0\}$. We now compute that for $k \in \mathbb{Z} \backslash\{0\}$ and $u \geq d \geq 0$ and $0 \leq s \leq u \wedge d^{*}$,

$$
\begin{gathered}
\frac{\left(1+\left|\lambda_{k}\right| u\right)^{1-\nu} s^{1-\nu}}{\left(1+\left|\lambda_{k}\right| d\right)^{(1-\nu) / 2}\left(1+\left|\lambda_{k}\right| s\right)^{(1-\nu) / 2} u^{1-\nu}} \leq\left(\frac{1}{u}+\left|\lambda_{k}\right|\right)^{1-\nu} \frac{s^{1-\nu}}{\left(1+\left|\lambda_{k}\right| s\right)^{(1-\nu) / 2}} \\
\leq\left(\frac{1}{s}+\left|\lambda_{k}\right|\right)^{1-\nu} \frac{s^{1-\nu}}{\left(1+\left|\lambda_{k}\right| s\right)^{(1-\nu) / 2}}=\left(1+\left|\lambda_{k}\right| s\right)^{(1-\nu) / 2} \\
\leq\left(1+\left|\lambda_{k}\right| d^{*}\right)^{(1-\nu) / 2}=\left|\lambda_{k}\right|^{(1-\nu) / 2}\left(\frac{1}{\left|\lambda_{k}\right|}+d^{*}\right)^{(1-\nu) / 2} \\
\leq\left|\lambda_{k}\right|^{(1-\nu) / 2}\left(\frac{1}{\left|\lambda_{1}\right|}+d^{*}\right)^{(1-\nu) / 2} .
\end{gathered}
$$

Thus there is a constant $C>0$ such that

$$
\left|\xi_{k}(d)\right| \leq C\left|\lambda_{k}\right|^{(1-\nu) / 2} \int_{u=d}^{\infty} \int_{s=0}^{u \wedge d^{*}} e^{\beta_{k} d-2 \beta_{k} u+\beta_{k} s} d s d u
$$


for all $d>0$ and $k \in \mathbb{Z} \backslash\{0\}$. If $d>d^{*}$, we compute that

$$
\int_{u=d}^{\infty} \int_{s=0}^{u \wedge d^{*}} e^{\beta_{k} d-2 \beta_{k} u+\beta_{k} s} d s d u \leq d^{*} e^{\beta_{k}\left(d+d^{*}\right)} \int_{u=d}^{\infty} e^{-2 \beta_{k} u} d u \leq d^{*} \frac{e^{-\beta_{k}\left(d-d^{*}\right)}}{\sqrt{2}\left|\lambda_{k}\right|}
$$

and if $d<d^{*}$, then

$$
\int_{u=d}^{\infty} \int_{s=0}^{u \wedge d^{*}} e^{\beta_{k} d-2 \beta_{k} u+\beta_{k} s} d s d u=d^{*} e^{\beta_{k} d} \int_{u=d}^{\infty} e^{-\beta_{k} u} d u=\frac{d^{*} \sqrt{2}}{\left|\lambda_{k}\right|} .
$$

Since $\beta_{k} \geq \beta_{1}$, we have that

$$
\begin{aligned}
& \int_{u=d}^{\infty} \int_{s=0}^{u \wedge d^{*}} e^{\beta_{k} d-2 \beta_{k} u+\beta_{k} s} d s d u \leq \frac{d^{*} \sqrt{2}}{\left|\lambda_{k}\right|} \exp \left[-\beta_{k}\left(\left(d-d^{*}\right) \vee 0\right)\right] \\
& \leq \frac{d^{*} \sqrt{2}}{\left|\lambda_{k}\right|} \exp \left[-\beta_{1}\left(\left(d-d^{*}\right) \vee 0\right)\right] \leq \frac{d^{*} \sqrt{2}}{\left|\lambda_{k}\right|} \exp \left[-\beta_{1}\left(d-d^{*}\right)\right] \\
&=\frac{d^{*} \sqrt{2} e^{\beta_{1} d^{*}}}{\left|\lambda_{k}\right|} e^{-B_{1} d}
\end{aligned}
$$

for all $d>0$ and all $k \in \mathbb{Z} \backslash\{0\}$. Combining this with (59), we get (57) for $i=0$.

Turning now to $\dot{\zeta}_{k}$, we use (58). From Lemma 10.2, we have that there is a $C>0$ such that

$$
\left|\frac{\dot{\zeta}_{k}(d)}{\zeta_{k}(d)}\right| \leq C \frac{\left|\lambda_{k}\right|^{2} d}{\left(1+\left|\lambda_{k}\right| d\right)} \leq C \frac{\left|\lambda_{k}\right|\left(1+\left|\lambda_{k}\right| d\right)}{\left(1+\left|\lambda_{k}\right| d\right)} \leq C\left|\lambda_{k}\right|
$$

for all $k \in \mathbb{Z} \backslash\{0\}$ and all $d>0$. On the other hand, we also have that there is a constant $C>0$ such that

$$
\begin{aligned}
\left|A_{k}(d)\right| \leq C \frac{\left(1+\left|\lambda_{k}\right| d\right)^{(1-\nu) / 2}}{d^{1-\nu} e^{\beta_{k} d}} \int_{s=0}^{d \wedge d^{*}} s^{1-\nu} \frac{e^{\beta_{k} s}}{\left(1+\left|\lambda_{k}\right| s\right)^{(1-\nu) / 2}} d s \\
\quad=C \int_{s=0}^{d \wedge d^{*}} e^{-\beta_{k} d+\beta_{k} s} \frac{\left(1+\left|\lambda_{k}\right| d\right)^{(1-\nu) / 2} s^{1-\nu}}{\left(1+\left|\lambda_{k}\right| s\right)^{(1-\nu) / 2} d^{1-\nu}} d s
\end{aligned}
$$

for all $k \in \mathbb{Z} \backslash\{0\}$ and $d>0$. For $0<s<d \wedge d^{*}$, we see that

$$
\begin{aligned}
& \frac{\left(1+\left|\lambda_{k}\right| d\right)^{(1-\nu) / 2} s^{1-\nu}}{\left(1+\left|\lambda_{k}\right| s\right)^{(1-\nu) / 2} d^{1-\nu}}=\left(\frac{1}{d}+\left|\lambda_{k}\right|\right)^{(1-\nu) / 2} \frac{s^{1-\nu}}{\left(1+\left|\lambda_{k}\right| s\right)^{(1-\nu) / 2} d^{(1-\nu) / 2}} \\
& \leq\left(\frac{1}{s}+\left|\lambda_{k}\right|\right)^{(1-\nu) / 2} \frac{s^{1-\nu}}{\left(1+\left|\lambda_{k}\right| s\right)^{(1-\nu) / 2} d^{(1-\nu) / 2}}=\frac{s^{(1-\nu) / 2}}{d^{(1-\nu) / 2}} \leq 1
\end{aligned}
$$

for all $k \in \mathbb{Z} \backslash\{0\}$. Thus

$$
\begin{aligned}
\left|A_{k}(d)\right| \leq C e^{-\beta_{k} d} \int_{s=0}^{d \wedge d^{*}} e^{\beta_{k} s} d s= & \frac{C \sqrt{2}}{\left|\lambda_{k}\right|} \exp \left[-\beta_{k} d+\beta_{k}\left(d \wedge d^{*}\right)\right] \\
=\frac{C \sqrt{2}}{\left|\lambda_{k}\right|} \exp \left[-\beta_{k}\left(\left(d-d^{*}\right)\right.\right. & \vee 0)] \leq \frac{C \sqrt{2}}{\left|\lambda_{k}\right|} \exp \left[-\beta_{1}\left(\left(d-d^{*}\right) \vee 0\right)\right] \\
& \leq \frac{C \sqrt{2}}{\left|\lambda_{k}\right|} \exp \left[-\beta_{1}\left(d-d^{*}\right)\right] \leq C \sqrt{2} e^{\beta_{1} d^{*}} \frac{e^{-\beta_{1} d}}{\left|\lambda_{k}\right|}
\end{aligned}
$$


for all $d>0$ and $k \in \mathbb{Z} \backslash\{0\}$. This and (60) imply that there is a $C>0$ such that

$$
\begin{aligned}
\left|\dot{\xi}_{k}(d)\right| \leq C\left(\frac{\left|\lambda_{k}\right|}{\left|\lambda_{k}\right|^{(1+\nu) / 2}}+\frac{1}{\left|\lambda_{k}\right|}\right) e^{-\beta_{k} d} & =C\left|\lambda_{k}\right|^{(1-\nu) / 2}\left(1+\frac{1}{\left|\lambda_{k}\right|^{(3-\nu) / 2}}\right) e^{-\beta_{k} d} \\
& \leq C\left|\lambda_{k}\right|^{(1-\nu) / 2}\left(1+\frac{1}{\left|\lambda_{1}\right|^{(3-\nu) / 2}}\right) e^{-\beta_{k} d}
\end{aligned}
$$

for all $d>0$ and $k \in \mathbb{Z} \backslash\{0\}$, which gives us the bound (57) for $i=1$.

Now define $\tilde{\Psi}$ by (47).

Proof of Proposition 6.5. Fix any multi-index $(\alpha, \beta)$ of order 2 or less. We have that

$$
\left|\left(D^{(\alpha, \beta)} \tilde{\Psi}\right)(\theta, d)\right| \leq\left(\frac{2 \pi}{\alpha(0)}\right)^{\alpha} \frac{8}{(n+1)^{2}} \sum_{k \in \mathbb{Z}}|k|^{\alpha}\left|\zeta_{k}^{(\beta)}(d)\right|\left|\phi_{k}\right|
$$

for $(\theta, d) \in \mathbb{R} \times(0, \infty)$ if this derivative exists. We now use (57) to compute that for some $C>0$,

$$
\begin{aligned}
\sum_{k \in \mathbb{Z}}|k|^{\alpha}\left|\zeta_{k}^{(\beta)}(d)\right|\left|\phi_{k}\right| & \leq C \sum_{k \in \mathbb{Z}}|k|^{\alpha}\left|\lambda_{k}\right|^{\beta-(\nu+1) / 2}\left|\phi_{k}\right| e^{-d / C} \\
\leq & C e^{-d / C}\left\{\sum_{k \in \mathbb{Z}}|k|^{6}\left|\phi_{k}\right|^{2}\right\}^{1 / 2}\left\{\sum_{k \in \mathbb{Z}}|k|^{2 \alpha-6}\left|\lambda_{k}\right|^{2 \beta-(\nu+1)}\right\}^{1 / 2} .
\end{aligned}
$$

Standard Fourier calculations reveal that there is a $C>0$ (which does such not depend on $\tilde{\Phi})$ such that

$$
\left\{\sum_{k \in \mathbb{Z} \backslash\{0\}}|k|^{6}\left|\phi_{k}\right|^{2}\right\}^{1 / 2} \leq C\|\tilde{\Phi}\|_{C^{4}(\mathbb{R} \times\{0\})} .
$$

For the relevant $\alpha$ and $\beta$ we have that

$$
\sum_{k \in \mathbb{Z} \backslash\{0\}} k^{2 \alpha-6}\left|\lambda_{k}\right|^{2 \beta-(\nu+1)}<\infty .
$$

Thus the derivatives in (61) exist as real numbers for all $(\theta, d) \in \mathbb{R} \times(0, \infty)$ and when $\alpha+\beta \leq 1$ the bounds of Proposition 6.5 hold. Having allowed for $\alpha+\beta \leq 2$, we also know that $\tilde{\Psi}$ is in $C^{2}$ and we can easily check that it satisfies the stated partial differential equation. We can also extract the stated behavior at $d=0$ from dominated convergence.

\section{REFERENCES}

1. Milton Abramowitz and Irene A. Stegun, Handbook of mathematical functions with formulas, graphs, and mathematical tables, For sale by the Superintendent of Documents, U.S. Government Printing Office, Washington, D.C., 1964. MR 29:4914

2. Richard F. Bass and Krzysztof Burdzy, Fiber Brownian motion and the "hot spots" problem, Duke Math. J. 105 (2000), no. 1, 25-58. MR 2001g:60190

3. A. N. Borodin, A limit theorem for the solutions of differential equations with a random right-hand side, Teor. Verojatnost. i Primenen. 22 (1977), no. 3, 498-512. MR 58:24546

4. A. N. Borodin and M. I. Freidlin, Fast oscillating random perturbations of dynamical systems with conservation laws, Ann. Inst. H. Poincaré Probab. Statist. 31 (1995), no. 3, 485-525. MR 96c:60073 
5. Stewart N. Ethier and Thomas G. Kurtz, Markov Processes: Characterization and Convergence, John Wiley \& Sons Inc., New York, 1986. MR 88a:60130

6. L. C. Evans, Partial Differential Equations, American Mathematical Society, Providence, RI, 1998. MR 99e:35001

7. S. N. Evans and R. B. Sowers, Pinching and twisting Markov processes, submitted.

8. Mark Freidlin and Matthias Weber, Random perturbations of nonlinear oscillators, Ann. Probab. 26 (1998), no. 3, 925-967. MR 99g:60098

9. - A remark on random perturbations of the nonlinear pendulum, Ann. Appl. Probab. 9 (1999), no. 3, 611-628. MR 2000j:60067

10. Mark I. Freidlin and Alexander D. Wentzell, Random perturbations of Hamiltonian systems, Mem. Amer. Math. Soc. 109 (1994), no. 523, viii+82. MR 94j:35064

11. I. I. Gihman, Concerning a theorem of N. N. Bogolyubov, Ukrain. Mat. Ž. 4 (1952), 215-219. MR 17:738g

12. Mark Goresky and Robert MacPherson, Stratified Morse Theory, Springer-Verlag, Berlin, 1988. MR 90d:57039

13. R. Z. Has'minskiı̌, Diffusion processes with a small parameter, Izv. Akad. Nauk SSSR Ser. Mat. 27 (1963), 1281-1300. MR 29:6530

14. - A limit theorem for solutions of differential equations with a random right hand part, Teor. Verojatnost. i Primenen 11 (1966), 444-462. MR 34:3637

15. - Stochastic processes defined by differential equations with a small parameter, Teor. Verojatnost. i Primenen 11 (1966), 240-259. MR 34:3636

16. A. I. Neĭshtadt, Probability phenomena due to separatrix crossing, Chaos 1 (1991), no. 1, 42-48. MR 92i:58161

17. Frank W. J. Olver, Asymptotics and special functions, A K Peters Ltd., Wellesley, MA, 1997, Reprint of the 1974 original [Academic Press, New York; MR 55:8655, MR 97i:41001

18. G. C. Papanicolaou and W. Kohler, Asymptotic theory of mixing stochastic ordinary differential equations, Comm. Pure Appl. Math. 27 (1974), 641-668. MR 51:4384

19. - Asymptotic analysis of deterministic and stochastic equations with rapidly varying components, Comm. Math. Phys. 45 (1975), no. 3, 217-232. MR 54:1382

20. G. C. Papanicolaou, D. Stroock, and S. R. S. Varadhan, Martingale approach to some limit theorems, Papers from the Duke Turbulence Conference (Duke Univ., Durham, N.C., 1976), Paper No. 6, Duke Univ., Durham, N.C., 1977, pp. ii+120 pp. Duke Univ. Math. Ser., Vol. III. MR 57:1669

21. Daniel Revuz and Marc Yor, Continuous Martingales and Brownian Motion, Springer-Verlag, Berlin, 1991. MR 92d:60053

22. Clark Robinson, Dynamical systems, second ed., CRC Press, Boca Raton, FL, 1999, Stability, symbolic dynamics, and chaos. CMP 2001:03

23. R. L. Stratonovich, Topics in the Theory of Random Noise. Vol. I: General Theory of Random Processes. Nonlinear Transformations of Signals and Noise, Gordon and Breach Science Publishers, New York, 1963, Revised English edition. Translated from the Russian by Richard A. Silverman. MR 28:1660

24. Daniel W. Stroock and S. R. Srinivasa Varadhan, Multidimensional Diffusion Processes, Springer-Verlag, Berlin, 1979. MR 81f:60108

25. G. Wolansky, Limit theorem for a dynamical system in the presence of resonances and homoclinic orbits, J. Differential Equations 83 (1990), no. 2, 300-335. MR 91b:58223

Department of Mathematics, University of Illinois at Urbana-Champaign, Urbana, ILLINOIS 61801

E-mail address: r-sowers@math.uiuc.edu 